\title{
An investigation of software vulnerabilities in open source software projects using data from publicly-available online sources
}

S. M. Monzur Murshed

A thesis submitted to the Faculty of Graduate and Postdoctoral Affairs in partial fulfillment of the requirements for the degree of

\author{
Master of Applied Science \\ in \\ Technology Innovation Management
}

Carleton University

Ottawa, Ontario

Copyright @ 2017 S. M. Monzur Murshed 
An investigation of software vulnerabilities in open source software projects using data from publicly-available sources

\section{Abstract}

Software vulnerabilities is an active area of research, but little is known about how publicly-observable properties of open source software projects and developer communities relate to the time taken to discover and fix vulnerabilities in the projects' software. This thesis examines that relationship using data harvested from online sources about a sample of 60 open source content management system (CMS) projects and 1268 vulnerabilities affecting the software produced by those projects. Combining project release histories with metrics from two online databases provided reliable proxy dates for vulnerability introduction and fix, but not discovery. Higher commit density (a proxy for project activity) was associated with shorter time of exposure. The lifecycle model, data collection workflow, and software scripts will enable researchers to replicate and extend this analysis, and the evidence-based recommendations provided here will enable improvements to the coverage, quality, access, and integration of online sources for project and vulnerability metrics. 
An investigation of software vulnerabilities in open source software projects using data from publicly-available sources

\section{Acknowledgements}

I would like to acknowledge my thesis supervisor, Professor Steven Muegge, for his patient and tireless guidance.

I would like to thank my daughter, Muntaha, and my wife, Gulshan, for their tolerance. 
An investigation of software vulnerabilities in open source software projects using data from publicly-available sources

Copyright (C) 2017 S. M. Monzur Murshed

\section{Table of contents}

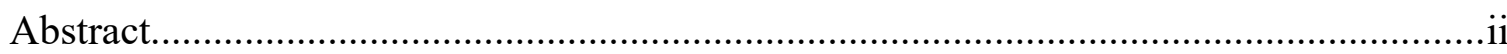

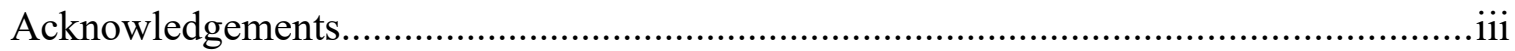

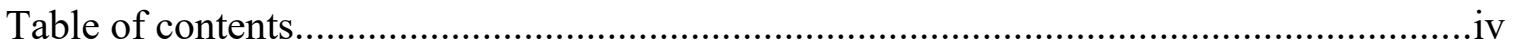

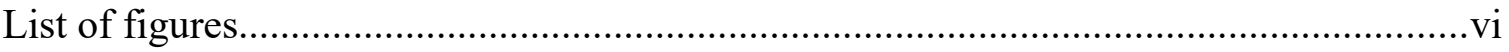

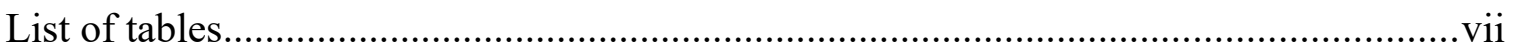

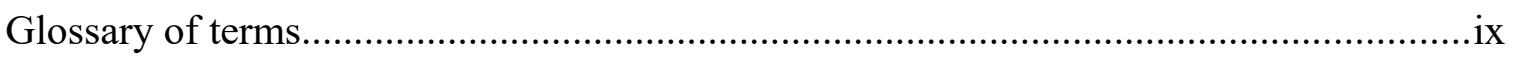

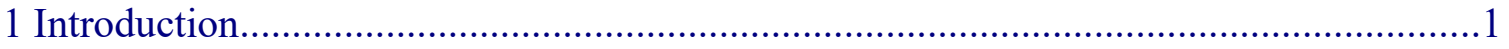

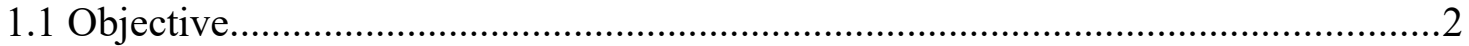

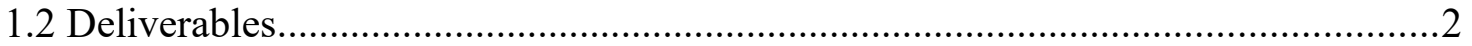

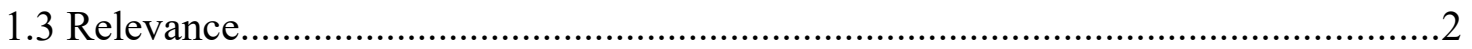

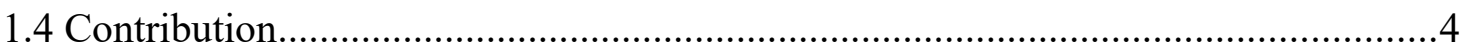

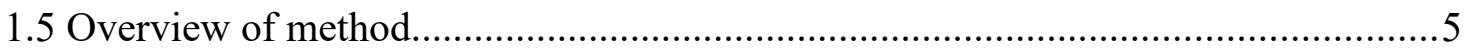

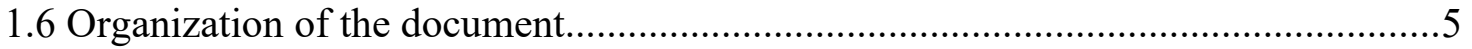

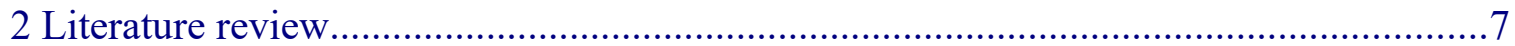

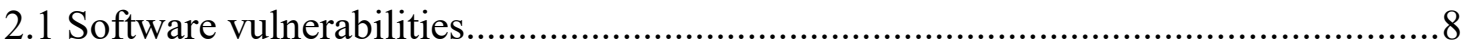

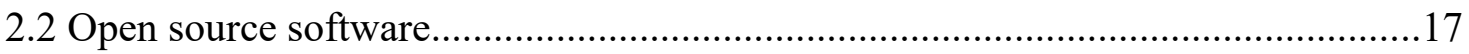

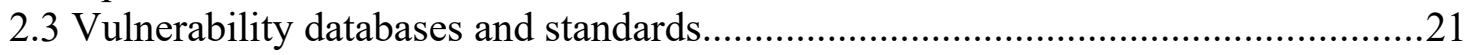

2.4 Summary and synthesis of key findings from the literature...............................24

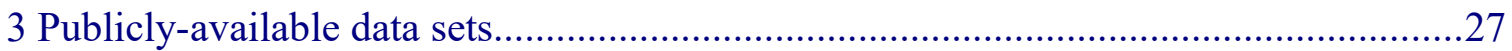

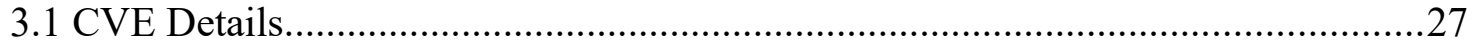

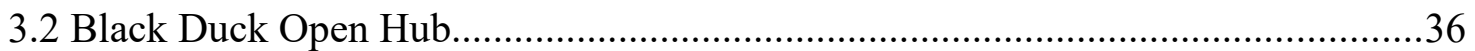

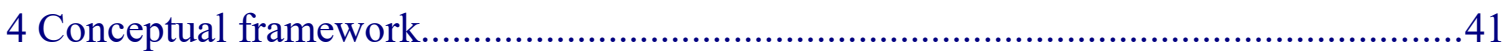

4.1 Software vulnerability ability lifecycle............................................................... 41

4.2 Application case: The Apache HTTP Server Project............................................44

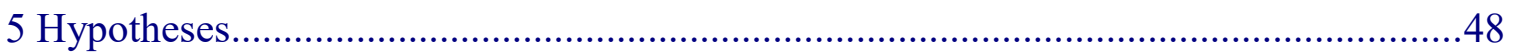

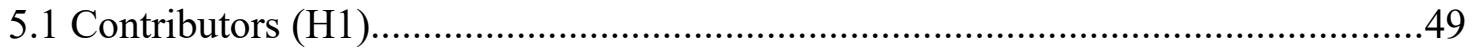

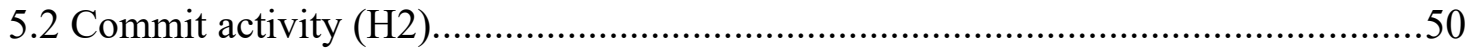

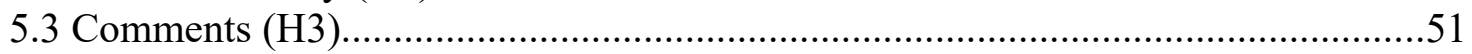

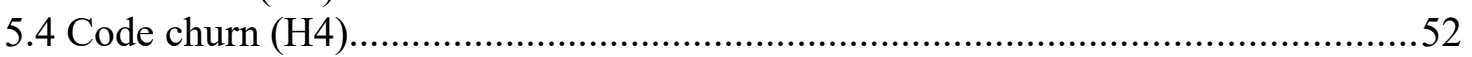

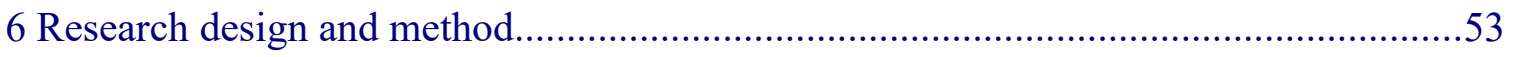

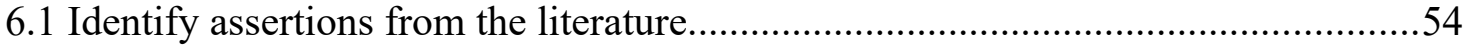

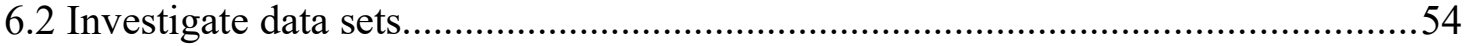

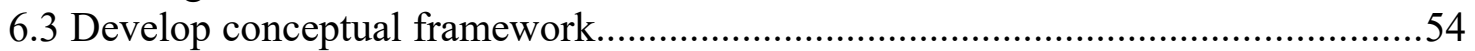

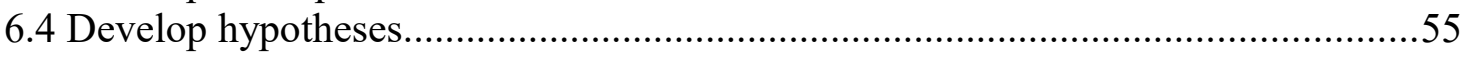

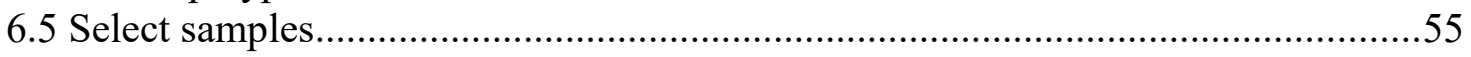

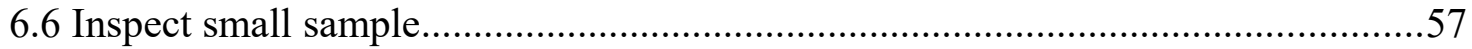

6.7 Specify variables and measures for full sample.............................................57 
An investigation of software vulnerabilities in open source software projects using data from publicly-available sources

Copyright (C) 2017 S. M. Monzur Murshed

6.8 Collect data for full sample. .60

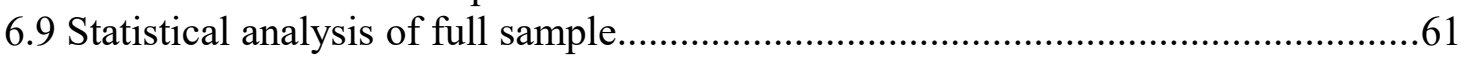

7 Results. .63

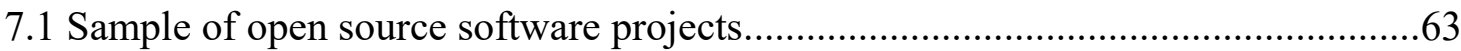

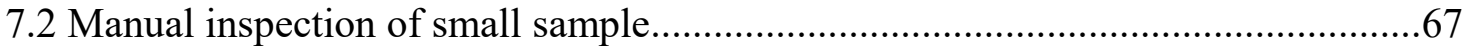

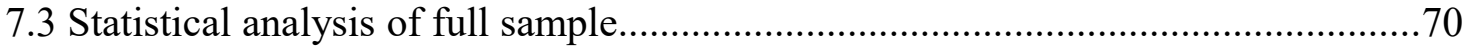

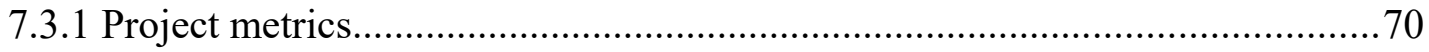

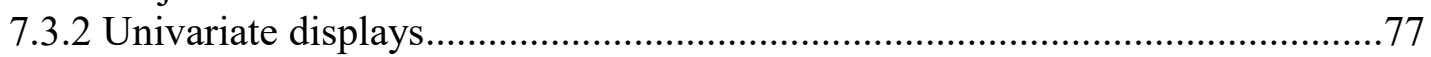

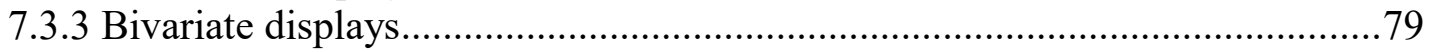

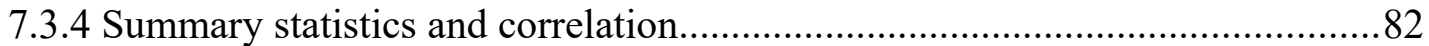

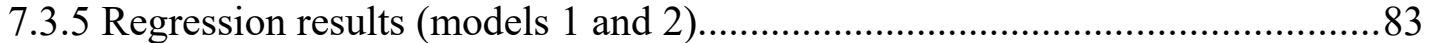

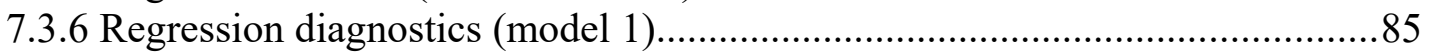

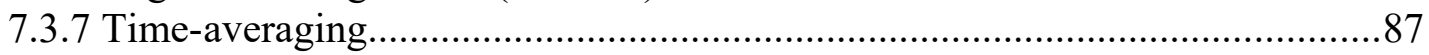

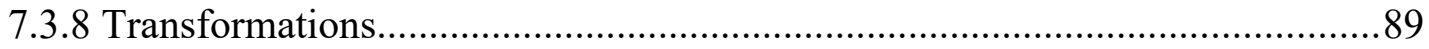

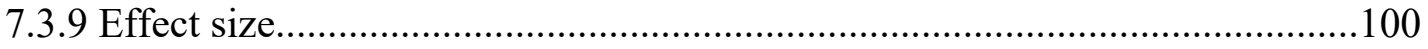

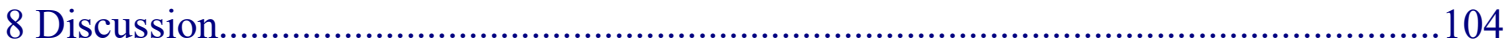

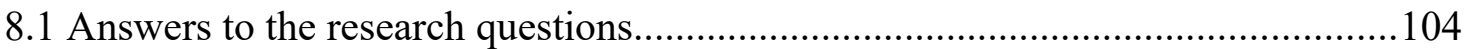

8.2 Lessons learned from writing this thesis......................................................... 108

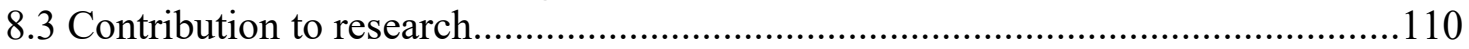

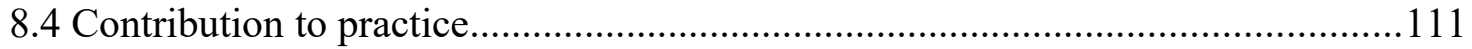

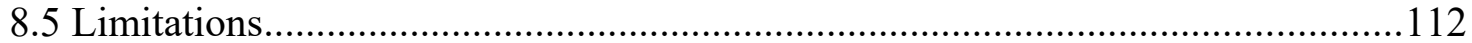

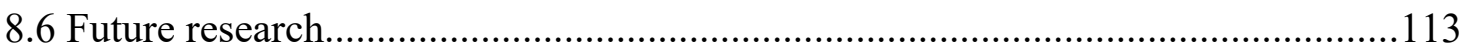

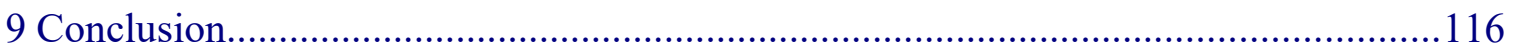

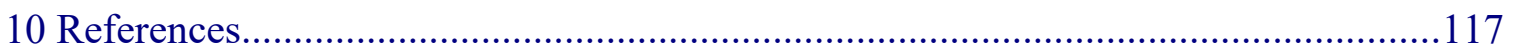

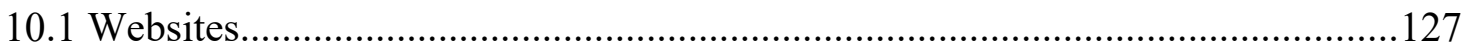

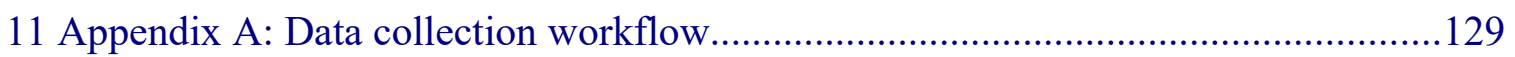

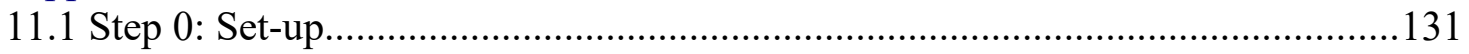

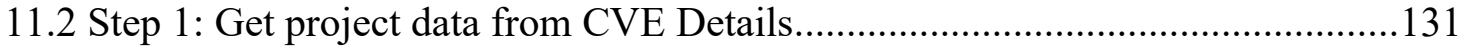

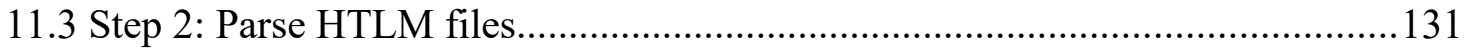

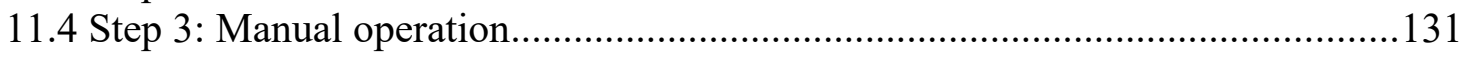

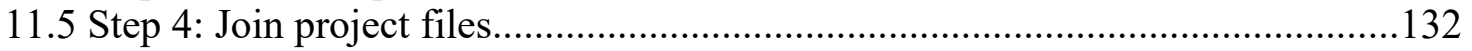

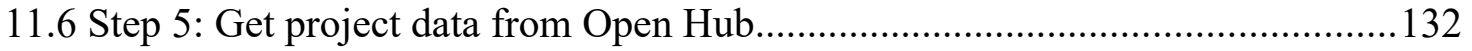

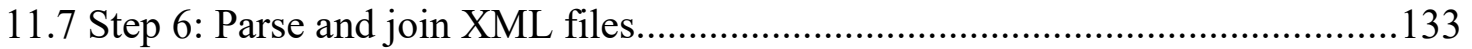

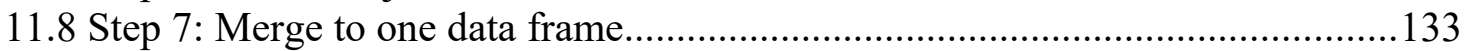

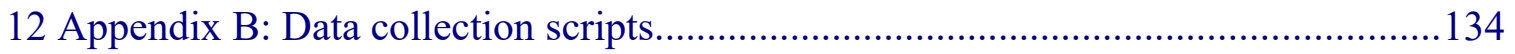

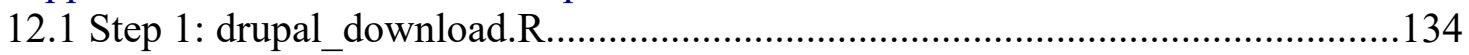

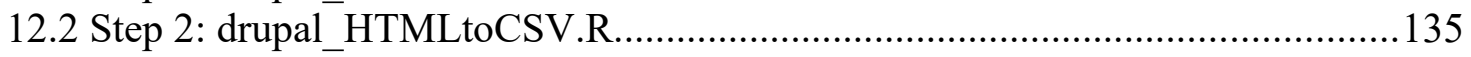

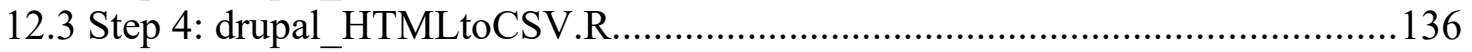

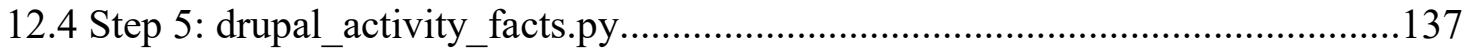


An investigation of software vulnerabilities in open source software projects using data from publicly-available sources

\section{List of figures}

Figure 1: Data aggregation by CVE Details.............................................................28

Figure 2: Sample response to an Open Hub API call (excerpt).....................................38

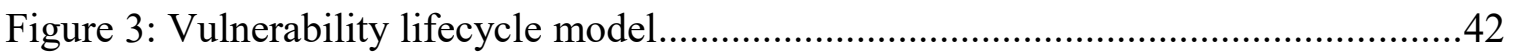

Figure 4: Timeline of vulnerabilities fixed in Apache HTTP Server 2.4.x.....................46

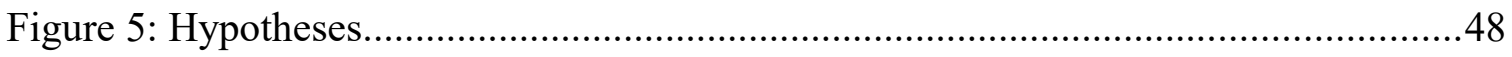

Figure 6: Project metrics of Plone (very large project)................................................73

Figure 7: Project metrics of Drupal (large project)..................................................... 74

Figure 8: Project metrics of MediaWiki (medium project)............................................75

Figure 9: Project metrics of Serendipity (small project).............................................76

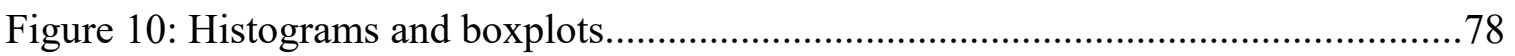

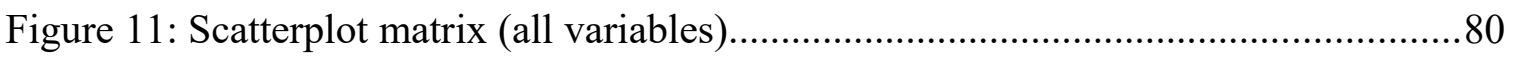

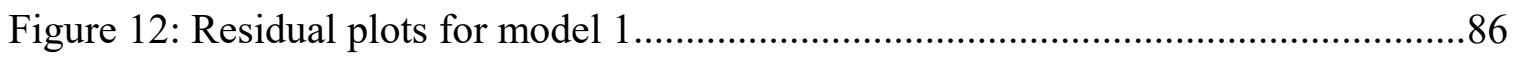

Figure 13: Parallel boxplots for transforms on model 4..............................................93

Figure 14: Influence plots of model 11 and $11^{*}$ (with 100 observations removed).........97

Figure 15: Influence plots of model 23 and 23* (with 100 observations removed).........98

Figure 16: Influence plots of model 27 and 27* (with 100 observations removed).........99

Figure 17: Data collection workflow 130 
An investigation of software vulnerabilities in open source software projects using data from publicly-available sources

Copyright (C) 2017 S. M. Monzur Murshed

\section{List of tables}

Table 1: Highlights of the scholarly and practitioner literature.......................................

Table 2: Definitions of "software vulnerability" in the literature...................................

Table 3: Definitional forms in the literature.............................................................. 10

Table 4: Vulnerability lifecycle models in the literature.............................................11

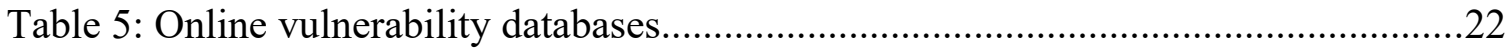

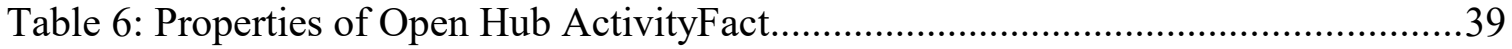

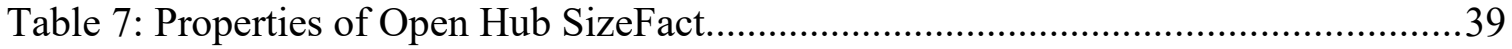

Table 8: Vulnerabilities fixed in Apache HTTP Server release 2.4.x............................45

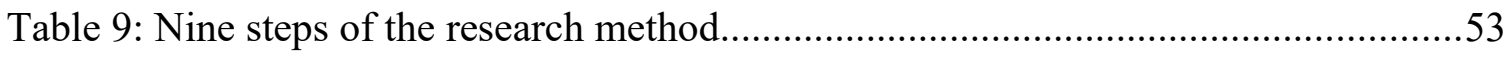

Table 10: Variables, measures, and sources for full sample analysis..............................58

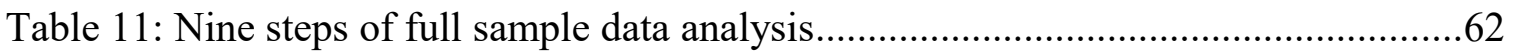

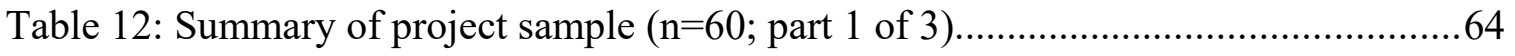

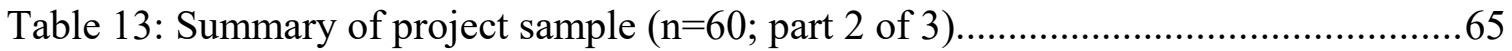

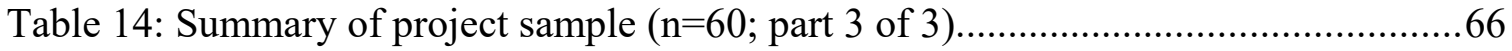

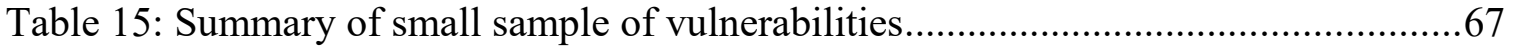

Table 16: Project metrics: mean, quartiles, and range.................................................. 71

Table 17: Projects sorted by size of code base (kLOC) .............................................. 72

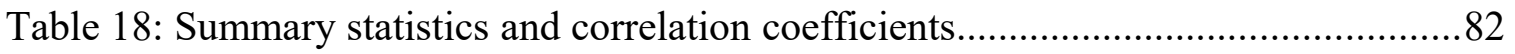

Table 19: Regression results (no time-averaging)...................................................... 83

Table 20: Regression results (all explanatory variables; time-averaging).......................88

Table 21: Regression results (significant variables only; time averaging)......................89

Table 22: Regression results (log transform of response variable) .................................92

Table 23: Regression results (Box-Cox power transform of response variable)..............92

Table 24: Regression results (spread-level power transform of response variable).........92

Table 25: Regression results (log transform of response variable; sig. variables only)....94

Table 26: Regression results (log transform of all variables).....................................95

Table 27: Regression results (log transform of significant variables only).....................95

Table 28: Regression results of Model 11* (with unusual observations removed)..........97

Table 29: Regression results of Model 23* (with unusual observations removed)..........98

Table 30: Regression results of Model 27* (with unusual observations removed)..........99 
An investigation of software vulnerabilities in open source software projects using data from publicly-available sources Copyright (C) 2017 S. M. Monzur Murshed

Table 31: Effect size of model 7 (linear model, 6-month time-averaging)...... 101

Table 32: Effect size of model 23 (log response, 6-month time-averaging)...... 102 
An investigation of software vulnerabilities in open source software projects using data from publicly-available sources

Copyright (C) 2017 S. M. Monzur Murshed

\section{Glossary of terms}

API:

CPE:

CVE:

CVRF:

CVSS:

CWE:

Exploit:

Free software:
Application programming interface, a set of clearly defined methods of communication between software components.

Common Platform Enumeration, a structured naming scheme and standard dictionary for information technology systems, software, and packages.

Common Vulnerabilities and Exposures, a dictionary of publicly known information security vulnerabilities and exposures, maintain

Common Vulnerability Reporting Framework, an XMLbased language that enables different stakeholders across different organizations to share critical security-related information in a single format. Maintained by the Industry Consortium for Advancement of Security on the Internet (ICASI).

Common Vulnerability Scoring System, an open framework for communicating the characteristics and severity of software vulnerabilities.

Common Weakness Enumeration, a standard dictionary of software weakness types.

Software code and/or sets of instructions to utilize a specific software vulnerability to cause harm. Exploits are often packaged and distributed as modules for the Metasploit framework of penetration testing software.

Software compliant with the Free Software Definition (FSD) to provide users with four essential freedoms: (0) the freedom to run the program for any purpose, (1) the freedom the study how the program works and change it, (2) the freedom redistribute copies to others, and (3) the freedom to distribute modified versions to others. 
An investigation of software vulnerabilities in open source software projects using data from publicly-available sources

Copyright (C) 2017 S. M. Monzur Murshed

HTML:

HyperText Markup Language, the standard markup language for creating web pages and web applications, and a cornerstone technology of the World Wide Web.

HTTP:

Hypertext Transfer Protocol, the protocol for data communication on the World Wide Web.

kLOC

Thousands of lines of code, a unit for comparing the size of a software project's source code base.

Open source software: $\quad$ Software compliant with the Open Source Definition (OSD), including access to the software source code, allowing modifications and derived works, and redistribution of modifications and derived works.

REST:

Representational state transfer, an approach to providing interoperability between web services; employed by many APIs.

Software vulnerability: A vulnerability in software. See "vulnerability."

URL:

Universal Resource Locator, a formal term for a web address, including web pages accessed through HTTP.

Vulnerability: $\quad$ A weakness that could be exploited to cause harm (Pfleeger et al. 2015; see also section 2.1).

XML:

Extensible Markup Language, markup language for encoding documents in a format that is both humanreadable and machine-readable, commonly used for data structures of web services. 
An investigation of software vulnerabilities in open source software projects using data from publicly-available sources

Copyright (C) 2017 S. M. Monzur Murshed

\section{Introduction}

Software vulnerabilities in open source software are receiving increasing attention, not just from security researchers and information technology professionals, but more recently from managers and entrepreneurs, and from the general public. The "Heartbleed" vulnerability in the Open SSL cryptographic software library disclosed in April 2014 (Bever, 2014; Perlroth, 2014a), the "Shellshock" family of vulnerabilities in the Unix Bash shell disclosed in September 2014 (Perlroth, 2014b), and the "POODLE" family of attacks on Version 3 of the Secure Socket Layer (SSL) protocol disclosed in October 2014 (Zetter, 2014; Möller et al. 2014) had extensive coverage in the popular press. The impacts are real: the Canada Revenue Agency shut down its websites for five days in April 2014 during the busiest month of the Canadian tax season following a confirmed breach using the Heartbleed vulnerability (CBC, 2014, 2015) Real-world vulnerability exploits feature with increasing prominence in the latest industry reports, including the Verizon Data Breach Investigation Report (DBIR; Verizon, 2016) and the Symantec Internet Security Threat Report (ISTR; Symantec, 2016).

Researchers are actively investigating the technical aspects of software vulnerabilities, and are collaborating with open source software organizations and government groups on applied research (e.g., Wheeler \& Khakimov, 2015). Nonetheless, the time taken to find and fix vulnerabilities in open source software projects has received comparably little attention. This apparent gap in the extant research is the motivation for this thesis. 


\subsection{Objective}

Examine, using publicly available data, the relationship between the project and community attributes of open source software projects and the time to discover and fix security vulnerabilities in the projects' software.

More specifically, this research addresses three guiding research questions:

(1) How do the observable properties of an open source software project relate to the time taken to discover and fix software vulnerabilities?

(2) To what extent is the data required to answer question 1 available through publiclyavailable online sources?

(3) How can existing publicly-available databases be improved?

\subsection{Deliverables}

There are four deliverables:

(1) a conceptual framework and a set of hypotheses developed from assertions in the scholarly and practitioner literature (chapters 4 and 5)

(2) results of hypotheses tests (chapter 7)

(3) a documented workflow and set of extensible software scripts to automate data collection from publicly-available online sources (Appendix A and Appendix B)

(4) a set of recommendations to improve existing databases of open source projects and software vulnerabilities (section 8.1)

\subsection{Relevance}

This research is relevant to at least three groups of stakeholders: (1) producers of open source software, (2) users and consumers of open source software, and 
(3) researchers in the domains of cybersecurity, open source software, strategy, management, and entrepreneurship.

The first stakeholder group is producers of open source software, including individual software developers and the management teams of firms sponsoring open source development. Software vulnerabilities require enormous amounts of effort, time, and money to find, publish, and fix (Shahazad et al., 2012). Actionable knowledge about finding and fixing vulnerabilities could free up the time and attention of experienced developers to work on other aspects of open source software projects, reduce the time and effort for core developers to review and approve outside contributions, and simplify the "on-boarding" of casual contributors becoming more active and productive within projects. In particular, the results here suggest that project leaders should seek to increase developer activity within the project, and that projects should provide accurate, complete, and accessible information about vulnerabilities fixed in each software release.

The second stakeholder group is users and consumers of open source software. A better understanding of how observable properties of open source software projects are related to software vulnerabilities could inform decisions by both individuals and organizations of which open source projects to use, consume, and potentially contribute back to (Williams \& Dabirsiaghi, 2012; Constantin, 2014; Wheeler \& Khakimov, 2015). In particular, the results here suggest that users and consumers should favour projects with more active developer communities.

The third group stakeholder group is researchers. Wheeler \& Khakimov (2015) write: "Measuring the security of software is a notoriously difficult and an essentially 
unsolved problem. Ideally we would identify metrics that directly determine whether or not an open source software project is producing secure software. However, since perfect metrics are not available, we are instead interested in metrics that provide some evidence that a project's product is more or less likely to be secure" (p. 2-1). In particular, the results here suggest that higher commit density is associated with shorter vulnerability exposure time - a desired property of secure software.

\subsection{Contribution}

This thesis makes three contributions to scholarship (developed in section 8.3):

1. Results of statistical hypothesis tests showing that higher commit density (a measure of developer activity) is associated with shorter vulnerability exposure time.

2. A software vulnerability lifecycle model that operationalizes events (and thus the time intervals between events) using release dates of affected and unaffected versions of the software. The author is aware of several alternative approaches in the scholarly literature, but no prior studies using this particular approach of software release date.

3. A workflow and set of software scripts for automated data collection of software vulnerability metrics and open source project metrics for use and extension by others.

In addition, this thesis makes at least two contributions to practice (developed in section 8.4):

1. Recommendations for improving publicly-available data sources (section 8.1).

2. Evidence of an association between higher commit density and shorter exposure time is of practical importance to producers of open source software, consumers of open source software, and security professionals. 


\subsection{Overview of method}

This is a quantitative hypothesis-testing research design. Each hypothesis formalizes an assertion from the scholarly or practitioner literature, specified using a conceptual framework of the software vulnerability lifecycle. The unit of analysis is a vulnerability in the software produced by an open source software project. The sample is 1286 vulnerabilities affecting the software produced by 60 open source CMS (content management system) projects. Automated data collection techniques (e.g., Munzert et al. 2015) harvest data from two online sources - Black Duck Open Hub (https://openhub.net) for properties of open source software projects, and CVE Details (https://www.cvedetails.com) for properties of software vulnerabilities. The analytic approach is linear regression using the $\mathrm{R}$ language for statistical computing ( $\mathrm{R}$ Core Team, 2016) and the techniques recommended by Fox (2016) and Fox \& Weisberg (2011).

\subsection{Organization of the document}

This thesis is organized as ten chapters, each structured into sections and subsections, followed by two appendices. Chapter 2, "Literature review," surveys the salient scholarly and practitioner literature on software vulnerabilities, open source software, and vulnerability databases and standards. Chapter 3, "Publicly-available data sets," examines the content of the two online databases used in this research: (i) CVE Details, a searchable repository of software vulnerability reports, and (ii) Black Duck Open $\mathrm{Hub}$, an aggregator of open source project metrics. Chapter 4, "Conceptual frameworks," develops a life-cycle model of a software vulnerability and applies the 
model to examine publicly-available information on the Apache HTTP server project.

Chapter 5, "Hypotheses," develops a set of hypotheses to be tested. Chapter 6, "Research design and method," describes the steps followed for data collection and analysis to test the hypotheses. Chapter 7, "Results," presents the outcomes of the research, including a manual investigation of a small sample of vulnerabilities and the regression analysis and hypothesis tests of the full sample. Chapter 8, "Discussion," discusses the results of the research, including answers to the research questions, lessons learned by the author from writing this thesis, the contribution to research, the contribution to management practice, limitations of the research design, and opportunities for further research. Chapter 9, "Conclusion," concludes the thesis, and Chapter 10 is a list of references.

Two appendices follow the body of the thesis. Appendix A, "Data collection workflow," describes the steps to reproduce the data set by rerunning the existing scrips, explains the operation of each script, and explains to how to modify or extend the scripts for future work. Appendix B, "Data collection scripts," provides the source code for the scripts that were documented in Appendix A. 
An investigation of software vulnerabilities in open source software projects using data from publicly-available sources

Copyright (C) 2017 S. M. Monzur Murshed

\section{Literature review}

This chapter reviews the salient findings from the scholarly and practitioner

literature in three streams: (1) software vulnerabilities, (2) open source software, and

(3) vulnerability databases and standards. Table 1 is a summary of the highlights of the

three streams and citations to key sources for each stream. The following three sections

review each stream, and the final fourth section is a summary and synthesis of the key

lessons from the literature, across all three streams, that are most relevant to this research.

Table 1: Highlights of the scholarly and practitioner literature

\begin{tabular}{|c|c|c|}
\hline Stream & Key highlights of the stream & Citations to key sources \\
\hline $\begin{array}{l}\text { Software } \\
\text { vulnerabilities }\end{array}$ & $\begin{array}{l}\text { - Definitions of software vulnerabilities } \\
\text { - Various life-cycle models; emphasis on disclosure } \\
\text { policies and decisions by a software vendor } \\
\text { (unclear to what extent results would generalize to } \\
\text { community-developed open source software) } \\
\text { - Factors associated with fewer and less severe software } \\
\text { vulnerabilities include lower complexity, secure } \\
\text { architectural designs, secure coding practices, access to } \\
\text { source code, more co-developers and beta-testers, lower } \\
\text { code churn, and more and better testing and testing tools }\end{array}$ & $\begin{array}{l}\text { Arbaugh (2000) } \\
\text { Marconato (2013) } \\
\text { Shahazad et. al. (2012) } \\
\text { Feng et al. (2016) }\end{array}$ \\
\hline $\begin{array}{l}\text { Open source } \\
\text { software }\end{array}$ & $\begin{array}{l}\text { - Various assertions are embedded in the open source } \\
\text { practitioner writing, but few of these practitioner } \\
\text { assertions have been tested empirically } \\
\text { - Typical characteristics of an open source software } \\
\text { development process include meritocracy, accessibility, } \\
\text { transparency, community roles, a committer selection } \\
\text { process, release management, project governance, } \\
\text { and coding guidelines for committers and contributors }\end{array}$ & $\begin{array}{l}\text { Raymond (1999) } \\
\text { Fogel }(2006 ; 2016) \\
\text { Meneely \& Williams }(2009 ; \\
2010) \\
\text { Free Software Foundation } \\
\text { (2014) } \\
\text { Wheeler \& Khakimov (2015) } \\
\text { Muegge (2011) }\end{array}$ \\
\hline $\begin{array}{l}\text { Software } \\
\text { vulnerability } \\
\text { databases and } \\
\text { standards }\end{array}$ & $\begin{array}{l}\text { - The Common Vulnerabilities and Exposures (CVE) } \\
\text { system is a standard way to identify and describe } \\
\text { software vulnerabilities; each receives a unique CVE ID. } \\
\text { - There are more than } 100 \text { vulnerability databases and } \\
\text { security advisories, most derived from CVE IDs } \\
\text { - Known limitations in the CVE data } \\
\text { - Various related vulnerability standards for description, } \\
\text { classification, assessing severity, and remediation }\end{array}$ & $\begin{array}{l}\text { Joh (2011) } \\
\text { Frei (2009) } \\
\text { Common Vulnerabilities and } \\
\text { Exposures (CVE) } \\
\text { National Vulnerability Database } \\
\text { (NVD) }\end{array}$ \\
\hline
\end{tabular}




\subsection{Software vulnerabilities}

There is no general consensus among researchers about the precise definition of "software vulnerability" (Krsul, 1998; Ozment, 2007; Frei, 2009; Joh, 2011). Table 2 is a summary of seventeen definitions found in the literature, and Table 3 (on the following page) sorts these definitions into broad themes according to form.

This thesis adopts the definition recommended by Pfleeger et al. (2015) - a software vulnerability is a weakness that could be exploited to cause harm, where harm is defined as a negative consequence realized when a vulnerability is exploited. 
Table 2: Definitions of "software vulnerability" in the literature

\begin{tabular}{|c|c|c|c|}
\hline & Definition & Source & Also cited by: \\
\hline & $\begin{array}{l}\text { A weakness in automated systems security procedures, } \\
\text { administrative controls, internal controls, etc., that could be } \\
\text { exploited by a threat to gain unauthorized access to information or } \\
\text { to disrupt critical processing }\end{array}$ & $\begin{array}{l}\text { Longly \& Shain } \\
\text { (1987) }\end{array}$ & $\begin{array}{l}\text { Shrivastava et al. } \\
(2015, \text { p. 199) }\end{array}$ \\
\hline & $\begin{array}{l}\text { Weaknesses that allow a threat to compromise the security } \\
\text { (confidentiality, integrity, or availability) of an asset }\end{array}$ & $\begin{array}{l}\text { Mayerfeld } \\
(1988)\end{array}$ & Joh (2011, p. 9) \\
\hline & The ability of an agent to cause an attack event & Snow (1988) & Joh $(2011$, p. 9) \\
\hline & $\begin{array}{l}\text { Achievable bad events, which implies that the protections against } \\
\text { them are nonexistent, insufficient, or insufficiently protected }\end{array}$ & $\begin{array}{l}\text { Lewis } \\
(1988)\end{array}$ & Joh (2011, p. 9) \\
\hline $5 \mathrm{~A}$ & A defect which enables an attacker to bypass security measures & $\begin{array}{l}\text { Schultz et al. } \\
(1990)\end{array}$ & $\begin{array}{l}\text { Shrivastava et al. } \\
(2015, \text { p. } 199)\end{array}$ \\
\hline & $\begin{array}{l}\text { A vulnerable system is an authorized state from which an } \\
\text { unauthorized state can be reached using authorized state } \\
\text { transitions; a vulnerability is a characterization of a vulnerable state } \\
\text { which distinguishes it from all non-vulnerable states }\end{array}$ & $\begin{array}{l}\text { Cheswick \& } \\
\text { Bellovin } \\
(1994)\end{array}$ & Joh $(2011$, p. 9) \\
\hline $7 \mathrm{~A}$ & $\begin{array}{l}\text { A weakness in a system that can be exploited to violate the } \\
\text { system's intended behavior }\end{array}$ & $\begin{array}{l}\text { De Ru \& Eloff } \\
(1996)\end{array}$ & $\begin{array}{l}\text { Roumani } \\
(2012, \text { p. } 3)\end{array}$ \\
\hline $\begin{array}{r}8 \mathrm{~A} \\
\mathrm{C} \\
\mathrm{s}\end{array}$ & $\begin{array}{l}\text { An instance of an error in the specification, development, or } \\
\text { configuration of software such that its execution can violate the } \\
\text { security policy }\end{array}$ & $\begin{array}{l}\text { Krsul } \\
(1998, \text { p. 10) }\end{array}$ & \\
\hline $\begin{array}{r}9 \mathrm{~T} \\
\mathrm{~S}\end{array}$ & $\begin{array}{l}\text { Technological flaw in an information technology product that has } \\
\text { security or survivability implications }\end{array}$ & $\begin{array}{l}\text { Arbaugh et al., } \\
\text { 2000: p. } 54\end{array}$ & \\
\hline $10 \begin{array}{r}\mathrm{A} \\
\mathrm{C} \\
\mathrm{C}\end{array}$ & $\begin{array}{l}\text { An instance of a mistake in the specification, development, or } \\
\text { configuration of software such that its execution can violate the } \\
\text { [explicit or implicit] security policy }\end{array}$ & $\begin{array}{l}\text { Ozment } \\
(2007, \text { p. } 7)\end{array}$ & \\
\hline $\begin{array}{r}11 \text { A } \\
a \\
0\end{array}$ & $\begin{array}{l}\text { A bug, flaw, behavior, output, outcome or event within an } \\
\text { application, system, device, or service that could lead to an implicit } \\
\text { or explicit failure of confidentiality, integrity, or availability }\end{array}$ & $\begin{array}{l}\text { Schiffman } \\
(2007)\end{array}$ & $\begin{array}{l}\text { Roumani } \\
(2012, \text { p. } 3)\end{array}$ \\
\hline $12 \mathrm{~s}$ & $\begin{array}{l}\text { Security flaws, defects, or mistakes in software that can be directly } \\
\text { used by a hacker to gain access to a system or network }\end{array}$ & $\begin{array}{l}\text { Wang et al., } \\
(2009)\end{array}$ & Joh (2011, p. 9) \\
\hline $\begin{array}{l}13 “ \\
\text { ir } \\
\text { tr }\end{array}$ & $\begin{array}{l}\text { "Weakness in an information system, system security procedures, } \\
\text { internal controls, or implementation that could be exploited or } \\
\text { triggered by a threat source" }\end{array}$ & $\begin{array}{l}\text { CNSS } 4009 \\
(2010, \text { p. } 81)\end{array}$ & $\begin{array}{l}\text { NISTIR } 7298 \\
(2013, \text { p. } 212)\end{array}$ \\
\hline $14 \begin{array}{r}V \\
n\end{array}$ & $\begin{array}{l}\text { Weakness in the security system which might be exploited by } \\
\text { malicious users causing loss or harm }\end{array}$ & $\begin{array}{l}\text { Pfleeger et al. } \\
(2015)\end{array}$ & $\begin{array}{l}\text { Joh }(2011, \text { p. } 9 \\
\text { previous edition) }\end{array}$ \\
\hline 15 A & $\begin{array}{l}\text { A mistake in software that can be directly used by a hacker to gain } \\
\text { access to a system or network }\end{array}$ & $\begin{array}{l}\text { MITRE } \\
(2016 b)\end{array}$ & $\begin{array}{l}\text { Various related } \\
\text { standards }\end{array}$ \\
\hline \multicolumn{4}{|c|}{$\begin{array}{l}16 \text { A hole or a weakness in the application, which can be a design flaw O } \\
\text { or an implementation bug, that allows an attacker to cause harm to } \\
\text { the stakeholders of an application. Stakeholders include the } \\
\text { application owner, application users, and other entities that rely on } \\
\text { the application. }\end{array}$} \\
\hline & $\begin{array}{l}\text { A weakness in a product that could allow an attacker to compromise } \\
\text { the integrity, availability, or confidentiality of that product. }\end{array}$ & $\begin{array}{l}\text { Microsoft } \\
\text { (no date) }\end{array}$ & \\
\hline
\end{tabular}


Table 3: Definitional forms in the literature

\begin{tabular}{|c|c|c|c|}
\hline Form & Count & "A vulnerability is a ..." & Sources \\
\hline \multirow[t]{2}{*}{ weakness } & 7 & weakness & $\begin{array}{l}\text { Longly \& Shain (1987) } \\
\text { Mayerfeld (1988) } \\
\text { De Ru \& Eloff (1996) } \\
\text { Pfleeger et al. (2015) } \\
\text { NIST (2006) } \\
\text { Microsoft (no date) }\end{array}$ \\
\hline & & hole or weakness & OWASP (2016) \\
\hline \multirow[t]{5}{*}{ mistake } & 6 & mistake & $\begin{array}{l}\text { Ozment (2007) } \\
\text { MITRE (2016b) }\end{array}$ \\
\hline & & flaw, defect, or mistake & Wang et al. (2009) \\
\hline & & error & Krsul (1998) \\
\hline & & flaw & Arbaugh et al. (2000) \\
\hline & & defect & Schultz et al. (1990) \\
\hline \multirow[t]{5}{*}{ various } & 1 & bug, flaw, behavior, output, outcome or event & Schiffman (2007) \\
\hline & 1 & characterization of an unauthorized state & Cheswick \& Bellovin (1994) \\
\hline & 1 & achievable bad events & Lewis (1988) \\
\hline & 1 & ability & Snow (1988) \\
\hline & 17 & & \\
\hline
\end{tabular}

Table 4 is a summary of seven software vulnerability lifecycle models found in the literature. The various models differ in composition (events, phases, or states), the number of components, the labels used, and the purpose for which the model was developed. None are perfectly suited for this research; section 4.1 revisits these ideas to develop a vulnerability lifecycle model for the purposes of this research. 
Table 4: Vulnerability lifecycle models in the literature

\begin{tabular}{|c|c|c|c|}
\hline Sources & $\begin{array}{l}\text { Parts of the lifecycle: } \\
\text { states, phases, events, etc. }\end{array}$ & $\begin{array}{l}\text { Factors impacting } \\
\text { vulnerability lifecycle }\end{array}$ & $\begin{array}{l}\text { Context: How used? } \\
\text { How used by others? }\end{array}$ \\
\hline $\begin{array}{l}\text { Arbaugh } \\
\text { et al. } \\
(2000)\end{array}$ & $\begin{array}{l}7 \text { states: } \\
\text { (1) birth, (2) discovery, } \\
\text { (3) disclosure, (4) correction, } \\
\text { (5) publicity, (6) scripting, } \\
\text { (7) death }\end{array}$ & $\begin{array}{l}\text { a) Community } \\
\text { discovery } \\
\text { b) Organization } \\
\text { business interest }\end{array}$ & $\begin{array}{l}\text { Applied to interpret incident data } \\
\text { on three case studies of software } \\
\text { vulnerabilities } \\
\text { Jones (2007) } \\
\text { Okamura et al. (2009) }\end{array}$ \\
\hline $\begin{array}{l}\text { Schneier } \\
(2000)\end{array}$ & $\begin{array}{l}5 \text { phases of a vulnerability } \\
\text { window of exposure: } \\
\text { (1) before discovery, } \\
\text { (2) after discovery but before } \\
\text { announcement, } \\
\text { (3) after announcement } \\
\text { (4) automatic exploit tool } \\
\text { published; popularized, } \\
\text { (5) vendor issues a patch and } \\
\text { users install patch }\end{array}$ & $\begin{array}{l}\text { a) Effectiveness of } \\
\text { vulnerability testing } \\
\text { b) Process of fixing } \\
\text { vulnerabilities }\end{array}$ & $\begin{array}{l}\text { Framework for discussion of } \\
\text { disclosure policy } \\
\text { Shrivastava et al. (2015): } \\
\text { Framework for a stochastic } \\
\text { vulnerability discovery model } \\
\text { (VDM) for vulnerability prediction } \\
\text { using differential equations }\end{array}$ \\
\hline $\begin{array}{l}\text { Rescorla } \\
(2005)\end{array}$ & $\begin{array}{l}6 \text { states: } \\
\text { (1) introduction, (2) discovery, } \\
\text { (3) private exploitation, } \\
\text { (4) disclosure, (5) public } \\
\text { exploitation and (6) fix release }\end{array}$ & $\begin{array}{l}\text { a) Whitehat discovery } \\
\text { b) Blackhat discovery }\end{array}$ & $\begin{array}{l}\text { Framework to measuring the rate } \\
\text { of vulnerability discovery from } \\
\text { empirical data }\end{array}$ \\
\hline $\begin{array}{l}\text { Frei et al. } \\
(2006)\end{array}$ & $\begin{array}{l}4 \text { events: } \\
\text { (1) discovery, (2) exploit, } \\
\text { (3) disclosure, (4) patch }\end{array}$ & & $\begin{array}{l}\text { Framework for analysis of } \\
\text { security advisories }(n=80,000)\end{array}$ \\
\hline $\begin{array}{l}\text { Frei } \\
(2009) \\
\text { Frei et al. } \\
(2009)\end{array}$ & $\begin{array}{l}6 \text { events between phases: } \\
\text { (1) creation, (2) discovery, } \\
\text { (3) exploit, (4) disclosure, } \\
\text { (5) patch availability, and } \\
\text { (6) patch installation }\end{array}$ & $\begin{array}{l}\text { a) Motivation of vendors } \\
\text { b) Motivation of } \\
\text { cyber-criminals } \\
\text { c) Vulnerability market } \\
\text { d) Government } \\
\text { e) Researchers }\end{array}$ & $\begin{array}{l}\text { Frei (2009): Case study } \\
\text { comparing Microsoft and Apple } \\
\text { regarding zero day patches and } \\
\text { over due patches } \\
\text { Frei et al. (2009): Analysis of } \\
\text { security bulletins ( } n=200,000)\end{array}$ \\
\hline $\begin{array}{l}\text { Shahzad } \\
\text { et al. } \\
(2012)\end{array}$ & $\begin{array}{l}3 \text { events: } \\
\text { (d) disclosure of the vulnerability } \\
\text { to the public, } \\
\text { (p) release of patch to neutralize } \\
\text { the threat, } \\
\text { (e) exploitation of the } \\
\text { vulnerability with a script virus, } \\
\text { tool or other breach, }\end{array}$ & $\begin{array}{l}\text { a) Exploitation behavior } \\
\text { of hackers } \\
\text { b) Patching behavior of } \\
\text { vendors } \\
\text { c) Discovery and } \\
\text { disclosure behavior of } \\
\text { independent } \\
\text { organizations }\end{array}$ & $\begin{array}{l}\text { Exploratory measurement study } \\
\text { of software vulnerabilities } \\
\text { ( } n=46,310 \text { vulnerabilities) } \\
\text { Employs association rule mining } \\
\text { to extract rules of exploitation } \\
\text { behavior of hackers and patching } \\
\text { behavior of vendors }\end{array}$ \\
\hline $\begin{array}{l}\text { Marconato } \\
\text { et al. } \\
(2013)\end{array}$ & $\begin{array}{l}4 \text { events: } \\
\text { (1) discovery of vulnerability, } \\
\text { (2) disclosure of vulnerability, } \\
\text { (3) release of patch, } \\
\text { (4) availability of exploit }\end{array}$ & $\begin{array}{l}\text { a) Behavior of attackers } \\
\text { b) Behavior of system } \\
\text { administrator }\end{array}$ & $\begin{array}{l}\text { Stochastic modelling of security } \\
\text { risks faced by an information } \\
\text { system in operation }\end{array}$ \\
\hline
\end{tabular}


These prior studies of vulnerability lifecycle events have examined a variety of topics. Arbaugh et al. (2000) examined the sequencing of events in three case studies, reporting that birth, discovery, and disclosure always occurred in order, but after disclosure the vulnerability can be further disclosed, made public, scripted or corrected in any order: "Automating a vulnerability, not just disclosing it, serves as the catalyst for widespread intrusions" (p. 57). Schneier (2000) was concerned with disclosure policy, arguing that neither full disclosure nor secrecy can "solve" computer security. Rescorla (2005) examined trends over time, concluding that available data does not show a clear improvement in software quality despite increased effort to find and patch security holes. Frei et al. (2006), Frei (2009), and Frei et al. (2009) examine the impact of various vendor behaviours. Anablagan (2009) examined the application of statistical prediction models from reliability engineering. Marconato et al. (2013) examined time intervals between discovery and disclosure dates, and between exploit availability and disclosure dates, specifying both intervals as beta distributions, and estimating model parameters. None of the prior vulnerability lifecycle studies summarized in Table 4 specifically examined the determinates of the time taken to discover and fix vulnerabilities.

Other prior research has identified various factors that may be related to software vulnerabilities.

Software begins with architecture, and Feng et al. (2016) argues that architectural flaws may be one of the significant underlying causes causes of the difficulty of fixing security issues. Although rigorous research in this area is limited, the authors present some empirical results suggesting that architectural flaws are strongly correlated with 
high rates of security bugs.

Software is implemented in source code, and Wheeler (2015) writes about secure coding practices - design and implementation guidelines for coding secure programs. Similarly, McGraw (2006) calls for "building security in" at the design stage, and Foreman (2009) argues for managing potential vulnerabilities to IT systems rather than reactively cleaning up after a violation. A new software patch could contain a new vulnerability (Beattie et al., 2006; US-CERT, 2009), and new releases of software that fix vulnerabilities in older versions could introduce new vulnerabilities (Rescorla, 2005).

There is a literature on software testing to discover vulnerabilities sooner. Merkow \& Raghavan (2012) advocate for early consideration of test cases and testing methods during architecture and coding. Kaner (1999) writes: "Testing and fixing can be done at any stage in the cycle. However, the cost of finding and fixing errors increases dramatically as development progress."

Researchers have examined the behaviours of the software vendor. There is an extensive literature on disclosure policies (Schneier, 2000) - how organizations and individuals developing software can and/or should respond to learning about a vulnerability in their software. Arbaugh et al. (2000, p. 54) writes: "The many levels of disclosure comprise a vast continuum of who was informed and what aspects of the vulnerability the disclosure revealed." Nizovtsev \& Thursby (2007), Cavusoglu et al. (2007), and Arora et al. (2008) employ game-theoretic models to explore disclosure trade-offs across a range of circumstances. Frei et al. (2006) and Mitra \& Ransbotham (2015) are large-sample empirical studies of vendor behavior. Ransbotham et al. (2012) 
examines the efficiency of the reward structures for security vulnerability reporting. Others have investigated vendor patch policies to disseminate fixes for vulnerabilities (Okamur et al. 2009; Frei, 2009). Ioannidis et al. (2012) examines vendor patch policies by developing a mathematical model of costly deployment of software patches and computing the optimal frequencies under varying conditions. August \& Tunca (2011) examine vendor liability, specifically the comparative effectiveness of three different policies: (1) making the vendor liable for damages, (2) making the vendor liable for patching costs, and (3) government imposed security standards.

There is a body of research around vulnerability prediction models that attempts to predict which parts of code are most likely to contain vulnerabilities and thus focus attention on the areas of highest risk (Shin et al. 2011). Much of this research focuses on technical aspects of the code - either component-level attributes such as source lines of code, alert density from static analysis tools, and code churn information (Gegick et al. 2008), or file headers and function call relationships between components (Neuhasue et al. 2007). Walden et al. (2009) examined vulnerability prediction at the project level using a security resource indicator (SRI) computed as the sum of four binary values: (i) existence of a security URL, (ii) existence of a security e-mail address, (iii) existence of a vulnerability list, and (iv) existence of secure development guidelines; the authors examined 14 open source PHP web applications, and also examined the relationship between three complexity metrics and vulnerabilities.

In one of the largest studies in this stream, Shin et al. (2011) examined whether software metrics for complexity, code churn, and developer activity - captured by the 
acronym "CCD" - can discriminate between vulnerable and neutral files and predict vulnerabilities. The study reports that CCD metrics can discriminate between vulnerable and neutral files and can predict vulnerabilities with $>85 \%$ probability of detection but with less than 3\% precision. Even with high numbers of false positives, the authors argue that $\mathrm{CCD}$ metrics can reduce the vulnerability inspection effort compared to a random selection of files. Shin \& Williams (2008a) and (2008b) report early results of this approach with smaller sets of metrics and data sets.

Several of the arguments from the vulnerability prediction literature may be relevant here. Regarding code complexity, Shin et al. (2011, p. 774) writes:

Security experts claim that complexity is the enemy of security (McGraw, 2006; Schneier, 2003). Complexity can lead to subtle vulnerabilities that are difficult to test and diagnose (Schneier, 2003), providing more chances for attackers to exploit. Complex code is difficult to understand, maintain, and test. Therefore, complex code would have a higher chance of having faults than simple code. Since attackers exploit the faults in a program, complex code would be more vulnerable than simple code.

McCabe (1976) argues that complex code is more difficult to understand, maintain, and test. Ferguson et al. (2010) argues that exploits hide in complexity; to keep something secure, you need to keep it simple.

Regarding code churn, Shin et al. (2011, p. 774) writes:

Code is constantly evolving throughout the development process. Each new change in the system brings a new risk of introducing a vulnerability (Nagappan \& Ball, 2005; Graves et al. 2000).

Regarding developer activity, Shin et al. (2011, p. 775) writes:

Software development is performed by development teams working together on a common project. Lack of team cohesion, miscommunications, or misguided effort can all result in security problems (McGraw, 2006). 
Lastly, there is a practitioner-driven descriptive literature about threats, incidents, and data breaches that utilize software vulnerabilities. According to the Verizon 2016 Data Breach Investigations Report (DBIR; Verizon, 2016), software vulnerabilities are one of three themes shared by many incident patterns. However, little is known about the role of vulnerabilities because this information is often not measured, or is missing or incomplete in the incident reports provided to Verizon: "We have numerous breaches where we can infer that some Common Vulnerabilities and Exposures (CVE) were used in order for the attack to advance... Unfortunately, we don't have a tremendous amount of CVE data in our corpus, either because it was not measured or was unable to be identified" (2016, p. 12). Two key findings from the 2016 DBIR may be relevant here:

- “Older vulnerabilities are still heavily targeted; a methodical approach that emphasizes consistency and coverage is more important than expedient patching" (p. 13).

- "Half of all exploitations happen between 10 and 100 days after the vulnerability is published, with the median around 30 days" (p. 14).

Symantec publishes a similar annual report, the 2016 Internet Security Threat Report (ISTR; Symantec Corporation, 2016).

Much of the literature of software vulnerabilities assumes closed-source software that is developed and distributed by independent software vendors or multinational IT firms - not the community-developed open source software that is the object of this research, and the topic of the next session. 


\subsection{Open source software}

Open source software refers to software distributed under open source licenses that allow users to use, modify, and redistribute the software, in compliance with the Open Source Definition (OSD) maintained by the Open Source Initiative (OSI). This is closely related to the four freedoms of the Free Software Definition (FSD) maintained by the Free Software Foundation (FSF): (0) the freedom to run the program for any purpose, (1) the freedom the study how the program works and change it, (2) the freedom redistribute copies to others, and (3) the freedom to distribute modified versions to others (Stallman, 1996). Some researchers employ hybrid labels that include both ideas of open and free, such as FOSS (Free and open source software), FLOSS (Free and libre open source software) or F/LOSS (Free/libre open source software). This research employs the simpler label of "open source software" and examines projects that comply with the OSD, while recognizing that all of the projects examined here are also free software in compliance with the FSD. The is similar to the approach taken by Wheeler \& Khakimov (2015) regarding the larger problem of securing open source software more generally. Advocates of free and open source software argue that the four freedoms are a precondition for secure computing: (i) vulnerabilities are more easily discovered; (ii) vulnerabilities that are discovered can be studied and exposed, (iii) fixes can be developed, and (iv) fixes can be distributed to others. Vulnerabilities include both "bugs" introduced by mistake and also deliberate additions of malicious features either by the vendor or by others. A statement from the Free Software Foundation explains this position (Free Software Foundation, 2014): 
Software freedom is a precondition for secure computing; it guarantees everyone the ability to examine the code to detect vulnerabilities, and to create new and safe versions if a vulnerability is discovered. Your software freedom does not guarantee bug-free code, and neither does proprietary software: bugs happen no matter how the software is licensed. But when a bug is discovered in free software, everyone has the permission, rights, and source code to expose and fix the problem. That fix can then be immediately freely distributed to everyone who needs it. Thus, these freedoms are crucial for ethical, secure computing.

Proprietary, (aka nonfree) software relies on an unjust development model that denies users the basic freedom to control their computers. When software's code is kept hidden, it is vulnerable not only to bugs that go undetected, but to the easier deliberate addition and maintenance of malicious features...

Free software cannot guarantee your security, and in certain situations may appear less secure on specific vectors than some proprietary programs. As was widely agreed in the aftermath of the OpenSSL "Heartbleed" bug, the solution is not to trade one security bug for the very deep insecurity inherently created by proprietary software - the solution is to put energy and resources into auditing and improving free programs.

John Sullivan, the Executive Director of the Free Software Foundation, writes about the impact and importance of the option to modify software (Sullivan, 2014, p. 2):

The mere existence of the option for people to inspect, modify, and share the software they use has an important effect which, if it became the norm, would cause a dramatic change in the behavior of software companies, and the character of software....

We do need everyone to have the right to modify software; we need everyone to have that potential. The fact that any given person around the world could step up and make a modification to a program to make it act differently - such as removing a back door installed by some company - is a powerful check against unethical control of individuals through software.

Fogel (2006) writes about the practice of developing open source software,

including starting a project, choosing a license, technical infrastructure (for

communication, version control, and bug tracking), social and political infrastructure

("governance" by meritocracy, election of new committers by the existing committers), commercial relationships, communications, release management, and "managing" 
volunteers. A second edition (Fogel, 2016), published online and maintained as a living document, demonstrates how the technical infrastructure is very different today from 2006, while the organizing principles have persisted with little change.

Raymond (1999) introduced many open source concepts that remain prominent:

- "Every good work of software starts by scratching a developer's personal itch" (rule $1)$.

- "Good programmers know what to write. Great ones know what to rewrite (and reuse)" (rule 2)

- "Treating your users as co-developers is your least-hassle route to rapid code improvement and effective debugging" (rule 6).

- "Release early. Release often" (rule 7).

- Linus's Law: “Given a large enough beta-tester and co-developer base, almost every problem will be characterized quickly and the fix obvious to someone. Or, less formally, 'Given enough eyeballs, all bugs are shallow" (rule 8).

The last assertion implies that projects with a larger developer community and a larger engaged user community (especially active beta testers) may be able to respond to vulnerabilities and issue fixes more quickly.

There is also a growing body of scholarly work about vulnerabilities in open source software. Meneely \& Williams $(2009,2010)$ and Datta et al. (2014) empirically examine Raymond's assertion of Linus's Law. Meneely et al. (2013) and Bosu et al. (2014) examine introduction of vulnerabilities in open source projects. Meneely et al. (2014) examines the association between code review metrics and vulnerabilities. 
Ransbotham (2010) examines exploitation of vulnerabilities in open source projects.

Bhattacharya et al. (2013) examines bug reports and fixes in open source mobile apps.

Likewise, there is a growing practitioner literature about vulnerabilities in open source software. According to Constantin (2014), developers mistakenly assume that libraries from long-running open-source projects are well-written and bug-free. In fact, third-party code may contain vulnerabilities, and these vulnerabilities have propagated into thousands of products:

The reality is that many open source projects, even the ones producing code that's critical to the Internet infrastructure, are often poorly funded, understaffed and have nowhere close to enough resources to pay for professional code audits or the manpower to engage in massive rewrites of old code.

Constantin speculates that vulnerabilities are more likely to occur and remain undiscovered in old code or code of low maturity, in projects with less rigorous testing or fewer testing tools, and too few developers.

According to some practitioner arguments, open source projects may be capable of responding to discoveries more quickly and more effectively than vendors of closed source software. Jim Zemlin, Executive Director of the Linux Foundation, offered the following remarks at the 2015 Linux Collaboration Summit (Kerner, 20015):

"In open source, we put our laundry out to air in the front yard."

"In these cases [of damaging security issues found recently in open source code] the eyeballs weren't really looking." Modern software security is hard because modern software is very complex, Zemlin said. But open source can provide a unique response to growing software complexity and the associated risk of security vulnerabilities. "We all have access and can all work together," Zemlin said. "Open source by its nature lets us have a collective response to a collective problem."

However, in an empirical comparison of 17 open source and closed source software 
packages, Schryen (2011) writes: "The analysis illustrates there is no empirical evidence that the particular type of software development is the primary driver of security. Rather, the particular policies of vendors determine the patching behavior" (p. 130). Comparing open and closed development practices may be asking the wrong question (Schryen \& Rich, 2010): "Open source and closed source software development do not significantly differ in terms of vulnerability disclosure and vendors' patching behavior, a phenomenon that has been widely assumed, but hardly investigated" (Schryen, 2011, p. 131).

\subsection{Vulnerability databases and standards}

Hundreds of databases track security-related issues for different software applications (Massacci \& Nguyen, 2010). Some are managed by governments, others by private security companies, or open security communities, or individuals. According to Joh (2011), the MITRE Corporation CVE format (explained below) is the de facto standard way of labelling and describing software vulnerabilities.

Table 5 is a summary of online vulnerability databases, the sponsoring organization, whether or not the database is formally certified as CVE compatible by MITRE Corporation, and a URL for the website. As of December 1 2016, there were 148 products and services of 81 organizations certified as CVE compatible. However, many of the databases in Table 5 not formally certified as CVE compatible do also report CVE records and utilize the CVE dictionary. 
Table 5: Online vulnerability databases

\begin{tabular}{|c|c|c|c|}
\hline Product Name & Sponsoring organization & $\begin{array}{l}\text { CVE } \\
\text { compatible? }\end{array}$ & URL \\
\hline $\begin{array}{l}\text { Netpower Network } \\
\text { Vulnerability Scanner } \\
\text { (NPNS) }\end{array}$ & $\begin{array}{l}\text { Beijing Netpower } \\
\text { Technologies Inc. }\end{array}$ & Yes & http://www.netpower.com.cn/ \\
\hline $\begin{array}{l}\text { World Laboratory of Bugtraq } \\
\text { (WLB) } 2\end{array}$ & CXSecurity & Yes & http://cxsecurity.com/wlb/ \\
\hline $\begin{array}{l}\text { DragonSoft Vulnerability } \\
\text { Database }\end{array}$ & $\begin{array}{l}\text { DragonSoft Security } \\
\text { Associates, Inc. }\end{array}$ & Yes & http://vdb.dragonsoft.com/ \\
\hline X-Force Database & $\begin{array}{l}\text { IBM Internet Security } \\
\text { Systems }\end{array}$ & Yes & http://xforce.iss.net/ \\
\hline $\begin{array}{l}\text { Vulnerability } \\
\text { Countermeasure Information } \\
\text { Database (JVN iPedia) }\end{array}$ & $\begin{array}{l}\text { Information-technology } \\
\text { Promotion Agency, Japan } \\
\text { (IPA) }\end{array}$ & Yes & http://jvndb.jvn.jp/en/ \\
\hline $\begin{array}{l}\text { National Vulnerability } \\
\text { Database (NVD) }\end{array}$ & $\begin{array}{l}\text { National Institute of } \\
\text { Standards and Technology } \\
\text { (NIST) }\end{array}$ & Yes & https://nvd.nist.gov/ \\
\hline Exploit Database & Offensive Security & Yes & https://www.exploit-db.com/ \\
\hline Secunia Website & Secunia & Yes & https://secunia.com/ \\
\hline Vigil@nce & Silicomp-AQL & Yes & http://vigilance.fr/ \\
\hline $\begin{array}{l}\text { SecurityFocus Vulnerability } \\
\text { Database }\end{array}$ & Symantec Corporation & Yes & http://www.securityfocus.com/ \\
\hline SecureCAST & SecureCAST & Yes & http://securecast.co.kr/ \\
\hline $\begin{array}{l}\text { WPScan Vulnerability } \\
\text { Database }\end{array}$ & WPScan & Yes & https://wpvulndb.com/ \\
\hline CVE Details & Serkan Özkan & No & http://www.cvedetails.com/ \\
\hline CERT/CC Advisories & $\begin{array}{l}\text { Software Engineering } \\
\text { Institute (SEI), Carnegie } \\
\text { Mellon University }\end{array}$ & No & http://www.cert.org/ \\
\hline $\begin{array}{l}\text { CERT Vulnerability Notes } \\
\text { Database (VU) }\end{array}$ & $\begin{array}{l}\text { Software Engineering } \\
\text { Institute (SEI), Carnegie } \\
\text { Mellon University }\end{array}$ & No & http://www.kb.cert.org/vuls/ \\
\hline Security Tracker & Security Tracker & No & http://securitytracker.com/ \\
\hline $\begin{array}{l}\text { US-CERT Cyber Security } \\
\text { Alert }\end{array}$ & $\begin{array}{l}\text { Department of Homeland } \\
\text { Security }\end{array}$ & No & https://www.us-cert.gov/ \\
\hline Security Lab & Positive Technologies & No & http://en.securitylab.ru/ \\
\hline CERT UK & UK Government & No & https://www.cert.gov.uk/ \\
\hline CERT-EU & The EU Institutions & No & http://cert.europa.eu/ \\
\hline
\end{tabular}


MITRE Corporation (2016c) describes CVEs as follows:

Common Vulnerabilities and Exposures (CVE) is a dictionary of common names (i.e., CVE Identifiers) for publicly known cybersecurity vulnerabilities. CVE's common identifiers make it easier to share data across separate network security databases and tools, and provide a baseline for evaluating the coverage of an organization's security tools. If a report from one of your security tools incorporates CVE Identifiers, you may then quickly and accurately access fix information in one or more separate CVE-compatible databases to remediate the problem.

CVE was launched in 1999. It is the "common enumeration" used by the U.S. National Vulnerability Database (NVD) and most other vulnerability databases (Joh, 2011).

Previously, security tools had each used their own databases and naming conventions. A CVE record includes a unique identifier (CVE ID), a description, references, and the date that the entry was created.

MITRE Corporation is the primary CVE Numbering Authority (CNA) that assigns CVEs to new vulnerabilities (MITRE, 2016b). As of June 2016, MITRE had approved 25 other CNAs (MITRE, 2016d): 4 third-party coordinators (CERT/CC, Distributed Weakness Filing Project, ICS-CERT, and JPCERT/CC), and 22 software vendors including proprietary vendors (Adobe, Apple, BlackBerry, Cisco, EMC, Google, Hewlett Packard Enterprise, HP Inc., IBM, Intel, Juniper, Micro Focus, Microsoft, Oracle, SGI, and Symantec), open source foundations (including the Apache Software Foundation, Debian GNU/Linux, FreeBSD, Mozilla, Red Hat, and Ubuntu), and sponsors of open source (Google for Chromium and the Android Open Source Project).

Other related standards include the Common Weakness Enumeration (CWE) for vulnerability type, the Common Platform Enumeration (CPE) for naming affected products, and the Common Vulnerability Scoring System (CVSS) for severity. 


\subsection{Summary and synthesis of key findings from the literature}

Seven insights from across the scholarly and practitioner literature are particularly relevant for this thesis. First, a software vulnerability can be defined as a weakness that could be exploited to cause harm (Pfleeger et al. 2015). The Common Vulnerabilities and Exposure (CVE) system is the reference standard for identifying and uniquely labelling publicly-known software vulnerabilities. Each vulnerability is assigned a unique identifier called a CVE ID, and each CVE ID receives a corresponding record in the National Vulnerability Database (NVD) - a publicly-accessible repository of standards-based vulnerability management data maintained by the U.S. National Institute of Standards and Technology (NIST). There are many vulnerability databases and security advisories, most of which employ CVE IDs and other NVD standards.

Second, vulnerabilities vary widely in severity: a severe vulnerability can be exploited for much harm, but low-severity vulnerabilities may result in little or no harm.

Third, there are multiple levels of security analysis, with multiple variables at each level. A software system may be hierarchical, with an architecture of nested software components, each of which may contain other software components, and each component may contain vulnerabilities. Actions taken at a component-level to introduce fewer vulnerabilities, to reduce the severity of vulnerabilities that are introduced, or to reduce the time taken to discover and fix vulnerabilities could all improve componentlevel security, but a severe vulnerability in a different component could compromise system-level security of the larger software system.

Fourth, the themes emphasized within extant research on software vulnerabilities 
include factors impacting vulnerability introduction and severity, policies for disclosing vulnerabilities, and the exploitation of vulnerabilities. The time to discover and fix vulnerabilities has received less attention.

Fifth, sources in the scholarly and practitioner literature assert various factors associated with software that is "more secure" (variously defined, mainly in the sense of introducing fewer and less severe vulnerabilities):

- Access to source code (Raymond, 1999)

- Software freedom (FSF, 2014; Sullivan, 2014): the capabilities to use, examine, and modify software, and to distribute modified versions

- Simplicity (Ferguson et al. 2010); low complexity (Schneier, 2003; Shin et al. 2011); code that is easy to understand (McCabe, 1976)

- "Many eyeballs" (Raymond, 1999) in various forms: more committers, more users that are co-developers, more beta-testers, and more users stressing the software in different ways (Meneely \& Williams, 2009, 2010; Datta et al. 2014; Meneely et al. 2014)

- Secure architectural designs with "architectural integrity" (Feng et al. 2016)

- Use and enforcement of secure software coding practices (McGraw, 2006; Merkow \& Raghavan, 2012; Pfleeger et al. 2015; Wheeler \& Khakimov, 2015)

- Use of more and better testing tools (Hewlett Packard, 2013; Constantin, 2014) and audit services (Perlroth, 2014a)

- Committers that value security (Walden et al. 2009)

Sixth, sources in the scholarly and practitioner literature likewise assert various 
factors associated with software that is "less secure" (variously defined, mainly in the sense of introducing more and higher severity vulnerabilities):

- No access to source code (FSF, 2014); proprietary distribution licenses that restrict use, examination of source code, modification, and/or redistribution

- Complexity - "the enemy of security" (Schneier, 2003; q.v., McCabe, 1976; Ferguson et al. 2010; Shin et al. 2011)

- Higher code churn (Graves et al. 2000; Nagappan \& Ball, 2005; Shin et al. 2011)

- Inadequate resources (Wheeler \& Khakimov, 2015)

- Poor coordination and communication between developers (Shin et al. 2011)

- Architectural flaws, especially unstable interfaces (Feng et al. 2016)

- Less experienced developers unfamiliar with secure coding (Wheeler, 2015) Seventh, advocates of free and open source software argue that the freedoms to use, examine, modify, and redistribute are a precondition to secure software (Free Software Foundation, 2014; Sullivan, 2014). According to the strongest form of this argument, software without these freedoms, including proprietary software with no access to source code, must be regarded as insecure.

This chapter has reviewed the scholarly literature on software vulnerabilities, open source software, and vulnerability databases and standards. The next chapter reviews the publicly-available data sets about software vulnerabilities and open source software projects. 
An investigation of software vulnerabilities in open source software projects using data from publicly-available sources

Copyright (C) 2017 S. M. Monzur Murshed

\section{Publicly-available data sets}

This chapter examines the content of two publicly-accessible online databases:

CVE Details (https://www.cvedetails.com) and Black Duck Open Hub

(https://openhub.net). Each database is described in its own section.

\subsection{CVE Details}

CVE Details is a website providing an easy-to-use web interface to CVE vulnerability data for corporate IT security professionals. According to the CVE Details website (accessed September 30, 2016), it was created by Serkan Özkan, a security consultant who "spent too many hours trying to find an easy to use list of security vulnerabilities." It emerged from beta testing in 2010 (https://archive.org, May 15 2010). The site employs an advertising business model with revenues from advertisers seeking to target their messages to security professionals.

The CVE Details database aggregates CVE vulnerability data from the official daily xml feeds of the National Vulnerability Database (NVD) along with other sources including the Offensive Security Exploit Database (https://www.exploit-db.com/), the Rapid7 Exploit Database (https://www.rapid7.com/db/modules/), Microsoft Security Bulletins (https://technet.microsoft.com/en-us/security/bulletins.aspx), and additional information from product vendors and comments submitted by users (Özkan, 2012). On August 1 2016, there were 77,635 CVE records in the database. Figure 1 illustrates the relationship between the CVE Details database, the National Vulnerability Database, and the CVE List at the CVE Website maintained by MITRE Corporation. 


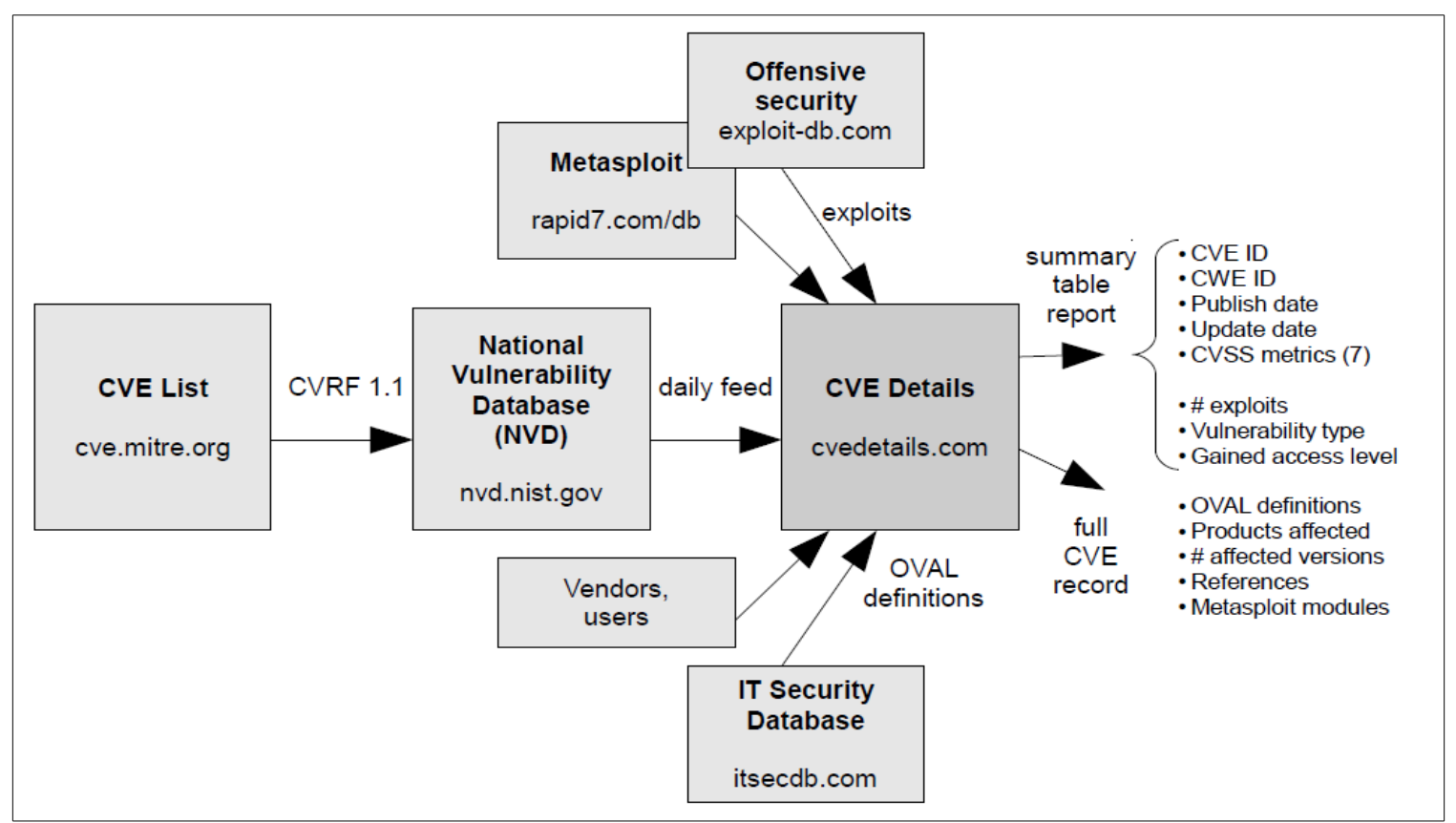

Figure 1: Data aggregation by CVE Details

The CVE Details database is browsable or searchable by various fields, including CVE ID, vendor, product, product version, vulnerability type, and date range. Search results are available in two main formats, summary table reports and individual CVE records, both also shown in Figure 1. Individual CVE records are displayed in a single web page. Reports of multiple CVE records are displayed in summary tables with one CVE ID per row. From a summary table report, a user can click through any CVE to view the individual CVE record. CVE Details can also provide various additional reports, including vulnerability trends over over time by product or by vendor.

A CVE Details summary table report includes fourteen fields for each CVE record:

- CVE ID: the unique identification number for the vulnerability. For example, the 
vulnerability known as "Heartbleed" (CBC News, 2014, 2015; Perlroth, 2014a;

Kerner, 2015) has a CVE ID of "CVE-2014-0160"

(https://www.cvedetails.com/cve-details.php?t=1\&cve_id=CVE-2014-0160).

- CWE ID: an identification number employing the Common Weakness

Enumeration (CWE) standard, a formal dictionary of software weakness types maintained by MITRE Corporation (https://cwe.mitre.org/). The Heartbleed vulnerability has a CWE ID of 119, corresponding to "Improper restriction of operations within the bounds of a memory buffer" (https://cwe.mitre.org/data/definitions/119.html).

- \# of Exploits: the number of known published exploits utilizing the vulnerability. The Heartbleed vulnerability has four published exploits. Although not shown in the summary table, from clicking through to the CVE record, the four exploits are exploit-db 32745 (https://www.exploit-db.com/exploits/32745/) and exploit-db 32764 (https://www.exploit-db.com/exploits/32764/) in the Offensive Security Exploit Database, and two Metasploit modules in the Rapid7 Exploit Database (https://www.rapid7.com/db/modules/auxiliary/scanner/ssl/openssl_heartbleed; https://www.rapid7.com/db/modules/auxiliary/server/openssl_heartbeat_client_m emory).

- Vulnerability Type(s): tags for thirteen categories of vulnerability, assigned by CVE Details using a combination of keyword matching algorithms and CWE IDs. The thirteen types are (1) denial of service (DoS), (2) code execution, (3) overflow, (4) cross-site scripting (XSS), (5) directory traversal, (6) bypass 
something, (7) gain information, (8) gain privileges, (9) SQL injection, (10) file inclusion, (11) memory corruption, (12) cross-site request forgery (CSRF), and (13) http response splitting. CVE Details does not explicitly define these type categories or details of its keyword matching algorithms, however all thirteen labels are familiar concepts commonly found in the penetration testing practitioner literature (e.g., Kim, 2014) and in sources on IT security (e.g., Pfleeger et al. 2015). The CVE Details website (accessed August 1 2016) advises that vulnerability type should be used as additional information and warns that it "may not be reliable." The Heartbleed vulnerability is tagged with the "overflow" and "gain information" vulnerability types.

- Publish Date: A date, not explicitly defined by CVE Details, that appears to match the "Date Entry Created" field of the CVE record in the MITRE Corporation CVE List, and the "Original release date" field of the CVE record in the National Vulnerability Database. The Heartbleed vulnerability has a Publish Date of "2014-04-07”.

- Update Date: A date, not explicitly defined by CVE Details, that appears to match the "Last revised" field of the CVE record in the National Vulnerability Database. The Heartbleed vulnerability has an Update Date of "2016-11-30".

- CVSS Score: A severity metric employing the Common Vulnerability Scoring System (CVSS), an open industry standard maintained for communicating the characteristics and severity of software vulnerabilities maintained by the Forum of Incident Response and Security Teams (FIRST; https://www.first.org/cvss). This 
field reports the CVSS "base score", an aggregation of six lower-level CVSS metrics (also reported by CVE Details, see below) intended to approximate the ease of exploit and the impact of exploit. Base score can range from 0 to 10, with 10 being the most severe. All seven CVSS metrics in CVE Details are aggregated from the National Vulnerability Database. The Heartbleed vulnerability has a CVSS Score of 5.

- Gained Access Level: The level of access that can be obtained by exploiting the vulnerability. The source for this metric is not stated at the CVE Details website; it may be user-submitted content, or it may be an automated outcome from keyword matching algorithms. The Heartbleed vulnerability has a Gained Access Level of "None."

- Access Vector: A CVSS metric of how a vulnerability may be exploited; can be local (L), adjacent network (A), or network (N). The Heartbleed vulnerability has an Access Vector of "Network Exploitable" (N).

- Access Complexity: A CVSS metric of how easy or difficult it is to exploit the discovered vulnerability; may be high (H), medium (M), or low (L). The Heartbleed vulnerability has an Access Complexity of "Low" (L; "Specialized access conditions or extenuating circumstances do not exist;. Very little knowledge or skill is required to exploit.”).

- Authentication: A CVSS metric of the number of times that an attacker must authenticate to a target to exploit it; may be multiple (M), single (S) or not required $(\mathrm{N})$. The Heartbleed vulnerability has an Authentication of "Not 
Required" (N; "Authentication is not required to exploit the vulnerability.").

- Confidentiality Impact, Integrity Impact, and Availability Impact: A set of three CVSS metrics of the impact of the vulnerability on the confidentially of data processed by the system, the integrity of the exploited system, and the availability of the target system, respectively; may be none $(\mathrm{N})$, partial $(\mathrm{P})$, or complete $(\mathrm{C})$. The Heartbleed vulnerability has impacts of "Partial" (P; "There is considerable informational disclosure."), "None" (N; "There is no impact to the integrity of the system."), and "None" (N; "There is no impact to the availability of the system."), respectively.

The terms "product" and "vendor" refer to the corresponding fields in the Common Platform Enumeration (CPE) dictionary (https://nvd.nist.gov/cpe.cfm), another related security standard maintained by the NIST National Vulnerability Database. The CPE dictionary is a structured naming scheme for information technology systems, software, and packages, where product refers to the IT artifact, and vendor refers to the organization producing the IT artifact. For some community-developed open source software projects, these fields may be the same. The Heartbleed vulnerability affects the OpenSSL software (product = "Openssl" in CPE and CVE Details), an open source software project maintained by the OpenSSL Foundation (vendor = "Openssl" in CPE and CVE Details). For other open source projects, these fields may be different. For example, the Apache HTTP Server (product = "Http Server") examined later in section 4.2, is maintained by the Apache Software Foundation (vendor = "Apache"), which hosts many open source software projects - 132 products in CVE Details with known 
vulnerabilities.

An individual CVE record in CVE Details includes five additional categories of information not reported in summary tables:

- Related OVAL Definitions: List of definitions (i.e., formal specifications) using the Open Vulnerability and Assessment Language (OVAL), a standard format used to specify what should be done to verify a vulnerability or a missing patch, maintained by the Center for Internet Security (https://oval.cisecurity.org/). The Heartbleed vulnerability has eight OVAL Definitions, each with a hyperlink to a corresponding database record in the IT Security Database (http://www.itsecdb.com/), an aggregator of OVAL definitions from multiple sources.

- Products Affected by CVE: List of products and version numbers using the CPE dictionary, including the first version affected by the vulnerability and the last version affected by the vulnerability. The Heartbleed vulnerability affected eleven different version of the Open SSL project, beginning with Open SSL version 1.0.1a and last affecting Open SSL version 1.0.2 Beta1.

- Number of Affected Versions By Product: Count of the number of product versions affected by the vulnerability, sorted by product. The Heartbleed vulnerability affected only one product - 11 vulnerable versions of the Open SSL project. Other vulnerabilities may affect multiple products.

- References for CVE: Links to websites with information about the vulnerability. May include bug reports, commits to a version control system, security 
advisories, exploit code, and blog posts and articles about solutions and tools. The Heartbleed vulnerability has approximately thirty-five references - an unusually large number because the this vulnerability received much high-profile attention. Between three and seven references is more typical, with some vulnerabilities having just one or even no references. Section 7.2 reports the results of a manual inspection of the references of twelve CVEs from the thesis data set. It reports that the references may contain voluminous information not captured in the fields of the CVE record or other security standards; however (1) formats vary widely between references, (2) the number of references varies widely between CVEs, (3) the quality of the references and the completeness of the reference inventory are unknown, and (4) judgement is needed to interpret information in each reference. Thus, large sample data collection from CVE references is difficult to automate, and is outside the scope of this theses.

- Metasploit Modules Related to CVE: Links to records in the Rapid7 Exploit Database to Metasploit modules that exploit the vulnerability. The Heartbleed vulnerability has two Metasploit exploit modules.

Although not reported in the summary table reports, these additional fields are available by clicking through to individual CVE records.

There are multiple ways to access the summary table reports and CVE records at CVE Details. First, one could use a web browser to visit the CVE Details website, browse the records or query an individual CVE or search on specific properties to produce a summary table report, and view or save the results. Second, one could write 
software scripts to formulate HTTP requests to the CVE Details website, and then

"screen scrape" or parse the HTML responses, also using software scripts. CVE Details

does not offer an API.

There are known limitations to the CVE Details database, many of which are inherited directly form upstream sources. From the CVE Details website (accessed

September 30, 2016):

CVE data have inconsistencies which affect accuracy of data displayed on www.cvedetails.com. For example a single product might have been defined with several different names. If a product is defined with different names in CVE data then they will be treated as different products by www.cvedetails.com. For example vulnerabilities related to Oracle Database $10 \mathrm{~g}$ might have been defined for products "Oracle Database", "Oracle Databaselog", "Databaselog", "Oracle 10g" and similar. Or a PHP vulnerability might have been defined for Fedora Linux 10, so number of vulnerabilities or statistics are only as accurate as CVE data. Please make sure that you manually verify all data before using.

Furthermore:

- Only exact versions listed as "vulnerable products" in NVD xml feeds are listed as vulnerable. Due to data inconsistencies some vulnerable versions may be missing. Vulnerable configurations listed for vulnerabilities are not always consistent with vulnerable software listed in CVE definitions.

- Vendor, product names and version numbers are not consistent in NVD feeds, for example some products are listed with several names like Adobe Reader, Adobe Acrobat Reader or IE and Internet Explorer. So some of the vulnerabilities are reported for IE while others are reported for Internet Explorer. Make sure that you manually verify that you have checked all possible names for a product.

- It's even worse for version, edition etc numbers.

The same limitations will affect all vulnerability databases based on NVD feeds.

In summary, CVE Details is the source used in this research for publicly-available information about security vulnerabilities. It reports standard metrics from the NIST National Vulnerability Database, the standard source for information about software 
vulnerabilities (Joh, 2011), aggregated with data from other sources. Data collection of summary reports can be conveniently automated with web scraping and text mining techniques from Munzert et al. (2015). Known limitations in the data set are similar to those of other vulnerability databases based on the National Vulnerability Database.

\subsection{Black Duck Open Hub}

Open Hub (full name Black Duck Open Hub) is a public directory of free and open source software. Originally founded in 2004 as Ohloh.net, it was acquired in 2009 by Geeknet (the owners of SourceForge, the largest hosting repository of open source software projects at that time; https://sourceforge.net/), then acquired again in 2010 by Black Duck Software, who then re-branded it as Black Duck Open Hub in 2014. Black Duck Software (https://www.blackducksoftware.com/), is the largest of three major providers of signature-based "clean IP" products and services - the others being Palamida (http://www.palamida.com/) and Protecode (http://www.protecode.com/; acquired by Synopsis in 2016; https://www.synopsys.com).

According to the Open Hub website (accessed August 2016), it offers "analytics and search services for discovering, evaluation, tracking, and comparing open source code and projects" comprising more than 21 billion lines of source code. It aggregates data harvested directly from various open source software forges (online collaborative facilities for developing and hosting projects, such as SourceForge, GitHub, and Gnu.org) and version control repositories (including Subversion, Git, CVS, Mercurial, and Bazaar).

By connecting to project source code repositories, analyzing both the code's history and ongoing updates, and attributing those updates to specific contributors, the Black Duck Open Hub can provide reports about the 
composition and activity of project code bases and aggregate this data to track the changing demographics of the FOSS world.

Additionally, Open Hub benefits from community contributions:

The Open Hub is editable by everyone, like a wiki. All are welcome to join, add new projects, and make corrections to existing project pages. This public review helps to make the Black Duck Open Hub one of the largest, most accurate, and up-to-date FOSS software directories available. We encourage contributors to join the Open Hub and claim their commits on existing projects and add projects not yet on the site. By doing so, Open Hub users can assemble a complete profile of all their FOSS code contributions.

Open Hub provides graphical data visualizations and reports about projects, people, and organizations, and comparative tools to compare projects (activity, code base, contributors), languages (based on commits, contributors, lines of code changes, and total number of new projects, and repositories (based on number of registered projects). The project records are of primary interest here.

Within a project record, Open Hub reports code (total lines of code, lines of comments, blank lines, programming languages used, and lines of code by language over the full history of the project), activity (commits per month, files modified, lines of code added, lines of code removed, and a complete commit history with contributor names), and community (number of contributors per month, top contributors, user ratings, and most recent contributors). Each project record also includes project summary, a quick reference dashboard with links to project resources, information about open source licenses, and metrics on project security.

The Ohloh API is a REST-based application programming interface to the Open Hub database. According to the Ohloh API documentation, "You can use the Ohloh API to create your own applications and web services based on Open Hub data" (Black Duck 
Software, 2014). Use of the API requires an Ohloh API key, available by registering with

Black Duck Software and agreeing to the Term of Use (http://blog.openhub.net/terms-2/).

Use of API keys is tracked, and normally limited to 1,000 requests per API key per day.

The API documentation includes examples of API calls in several programming languages, including Python, Ruby, Perl, Java, C++, bash, and PHP. Figure 2 is an example of an XML-formatted response returned by an HTTP GET request.

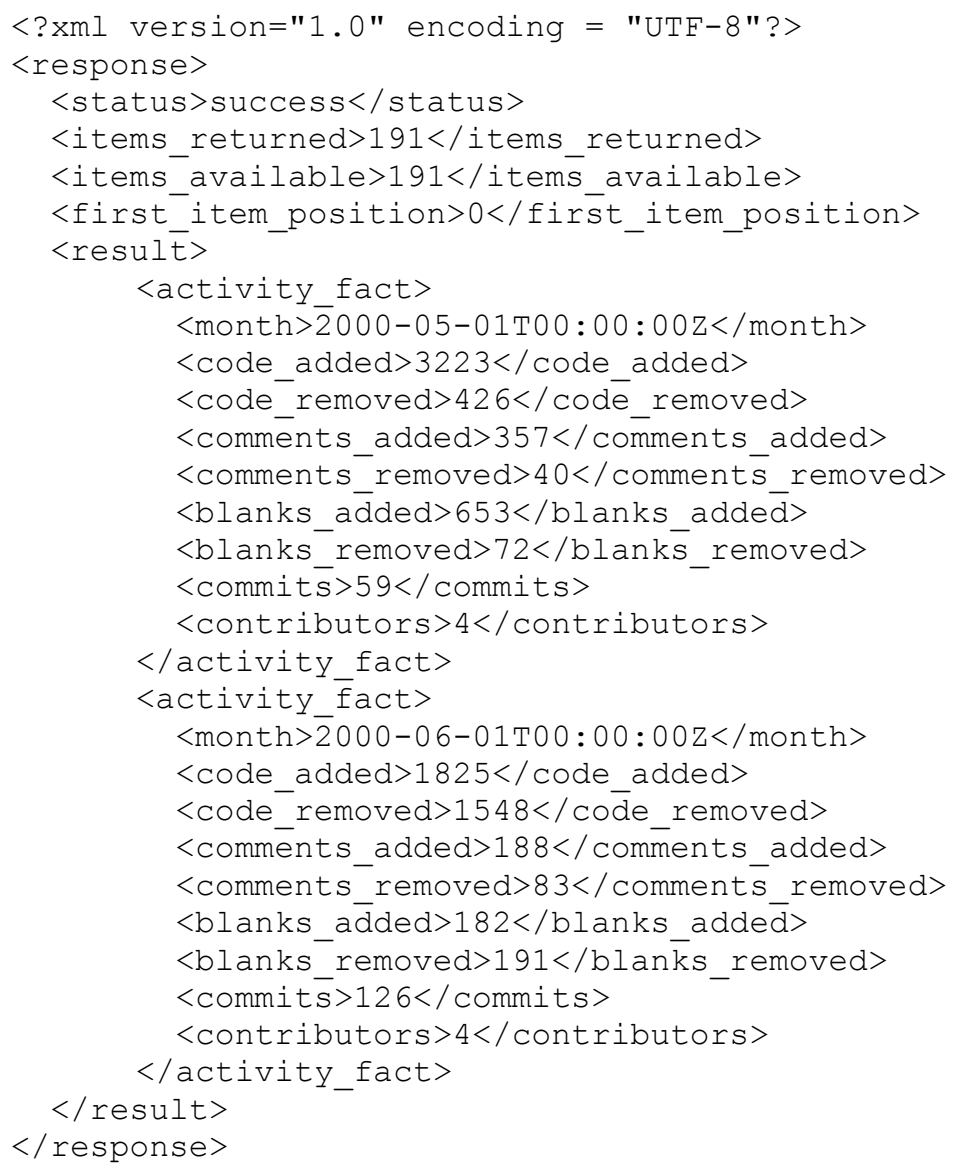

Figure 2: Sample response to an Open Hub API call (excerpt) 
The Ohloh API provides pre-computed collections of statistics about project

source code. An ActivityFact summarizes changes to lines of code, commits, and contributors in a single month, comprising the nine properties described in Table 6. A SizeFact provides monthly running totals of lines of code, commits, and developer effort, comprising the seven properties described in Table 7.

Table 6: Properties of Open Hub ActivityFact

\begin{tabular}{|c|c|c|}
\hline & Property & Description \\
\hline 1 & month & $\begin{array}{l}\text { Indicates the month covered by this ActivityFact. Only the year and month fields } \\
\text { are significant. This ActivityFact record includes all development activity that } \\
\text { occurred during this month. }\end{array}$ \\
\hline 2 & code_added & $\begin{array}{l}\text { The total new lines of code added, excluding comments and blanks, during this } \\
\text { month. }\end{array}$ \\
\hline 3 & code_removed & $\begin{array}{l}\text { The total lines of code removed, excluding comments and blanks, during this } \\
\text { month. }\end{array}$ \\
\hline 4 & comments_added & The total lines of new comments added during this month. \\
\hline 5 & comments_removed & The total lines of comments removed during this month. \\
\hline 6 & blanks_added & The total blank lines added during this month. \\
\hline 7 & blanks_removed & The total blank lines removed during this month. \\
\hline 8 & commits & The number of commits made during this month. \\
\hline 9 & contributors & The number of contributors who made at least one commit during this month. \\
\hline
\end{tabular}

Table 7: Properties of Open Hub SizeFact

\begin{tabular}{lll}
\hline Property & Description \\
\hline 1 month & $\begin{array}{l}\text { Indicates the month covered by this SizeFact. Only the year and month fields are } \\
\text { significant. This SizeFact record includes all development activity that occurred } \\
\text { during this month. }\end{array}$ \\
\hline 2 code & $\begin{array}{l}\text { The total net lines of code, excluding comments and blanks, as of the end of this } \\
\text { month. }\end{array}$ \\
\hline 3 comments & The total net lines of comments as of the end of this month. \\
\hline 5 & blanks & The total net blank lines as of the end of this month. \\
\hline 6 & commits & $\begin{array}{l}\text { The fraction of net lines which are comments as of the end of this month. } \\
\text { month. }\end{array}$ \\
\hline 7 & man_months & $\begin{array}{l}\text { The cumulative total months of effort expended by all contributors on this project, } \\
\text { including this month. For instance, if } 1 \text { contributor has worked for } 3 \text { months and } 2 \\
\text { developers have each worked for } 5 \text { months, man_months will be 13. This is the } \\
\text { running total of the ActivityFact contributors property. }\end{array}$ \\
\hline
\end{tabular}


Of the known limitations to the Open Hub database and the API documentation, three are most relevant to this thesis. First, Open Hub will aggregate any errors from its data sources, which include a variety of different software repositories that may differ in metrics collection and reporting. Second, no $R$ API libraries or examples of $R$ scripts are provided in the Ohloh API documents; rather than develop new $R$ code for accessing the Ohloh API, this thesis instead develops a set of Python scripts adapted from the Ohloh API code samples, which introduces a second scripting language to the data collection process (which otherwise employs $R$ exclusively). Third, the pre-computed ActivityFact and SizeFact bundles are conveniently accessed and processed, but are a partial view of the database.

In summary, Black Duck Open Hub is the source used in this research for publicly-available information about open source software projects. It aggregates data harvested directly from project source code repositories, and provides analytics about code, activity, and community. Data collection of project metrics can be can conveniently automated using the Ohloh API and scripts written Python, adapted from the examples in the Ohloh API documentation.

This chapter has examined the content, access, and limitations of the two publicly-accessible online databases used in this research for data collection about software vulnerabilities and open source software projects. The next chapter develops a conceptual framework of the software vulnerability lifecycle and both demonstrates and validates the framework using publicly-available data on an open source software project. 
An investigation of software vulnerabilities in open source software projects using data from publicly-available sources

Copyright (C) 2017 S. M. Monzur Murshed

\section{Conceptual framework}

This chapter develops a software vulnerability lifecycle model that is employed throughout the thesis. The chapter is structured in two sections. The first section describes and explains the lifecycle model. The second section applies the vulnerability lifecycle model to examine vulnerabilities in a popular open source software project - the Apache HTTP Server project.

\subsection{Software vulnerability ability lifecycle}

Table 4 in section 2.1 previously summarized the various software vulnerability lifecycle models found in the published literature. These models were developed or adapted for a variety of purposes, including discussion of policy (Schneier, 2000; Rescorla, 2005), interpreting case data (Arbaugh et al. 2000; Frei, 2009), developing mathematical models (Marconato, 2013; Shrivastava et al. 2015), and analysis of security advisories (Frei, 2006; Frei et al. 2000). Most of the models feature exploitation and attacker behavior which is not relevant here, and most applications of the models assume proprietary vendor-developed software rather than the community-developed open source software of interest here. Further, most of the models begin at discovery, with the introduction of the vulnerability to the software system as either absent from the model or outside the scope of the research in the sense that the introduction remains unknown.

Figure 3 is a graphical representation of the simplified lifecycle model employed here for this research. The model is comprised of three events and three time intervals. It begins at the introduction (i.e., the birth or creation) and is silent about exploitation. 


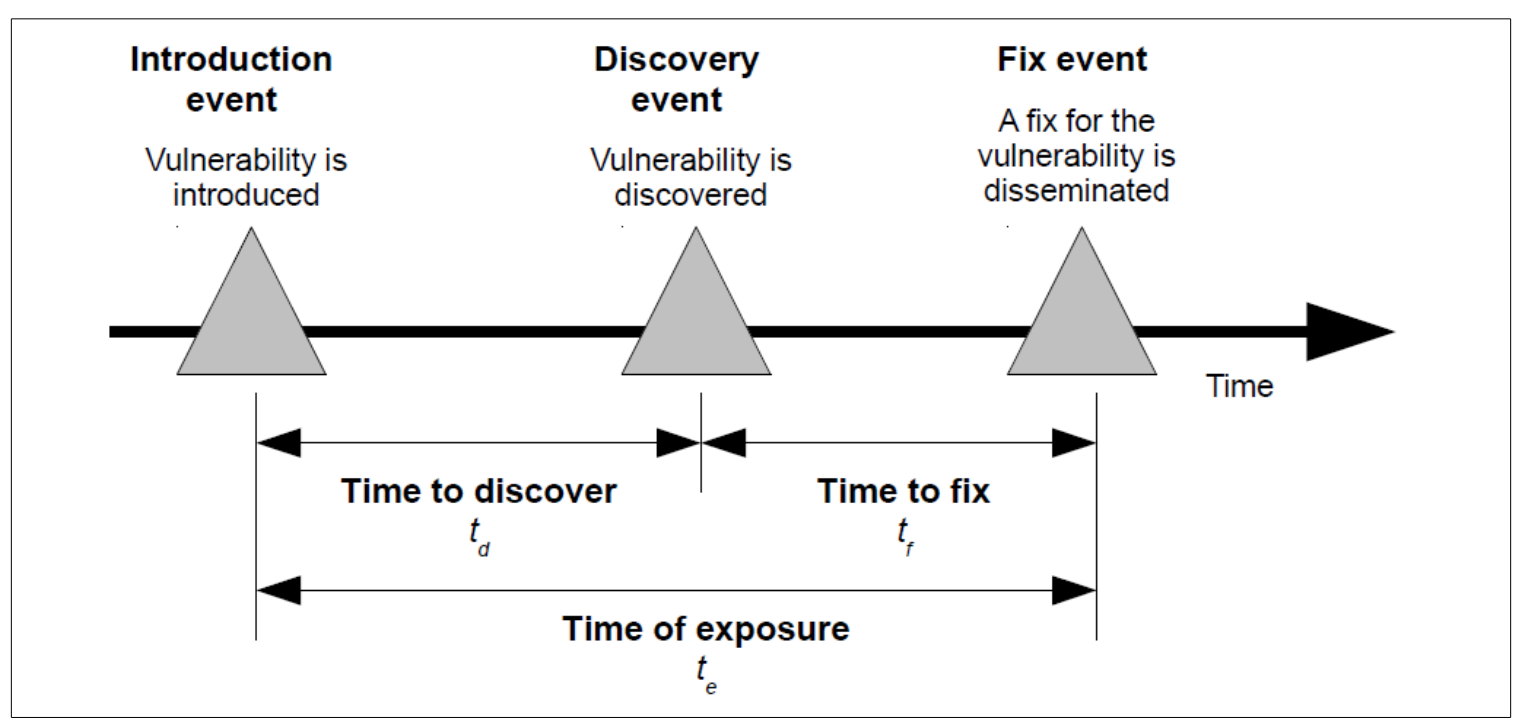

Figure 3: Vulnerability lifecycle model

The three events are (1) introduction of the vulnerability, (2) discovery of the vulnerability, and (3) dissemination of a fix to the vulnerability. Each event is specified by the date on which the event occurred.

The introduction event is the earliest date on which a user of the software could be affected by a new vulnerability. The vulnerability may exist previously in patches submitted to a bug-tracker, or in source code repositories, or in "unstable" developer builds, but is not introduced to the field until a version of the software including the vulnerability is packaged and released for use outside the developer community.

The discovery event is the earliest date on which the developers of the software become aware of the vulnerability and could potentially begin work on a fix. The vulnerability may previously be known to others outside the developer community (and perhaps exploited maliciously for harm), but is not discovered in this model until known to developers. Discovery could occur because the vulnerability was first identified by a 
developer, or because the vulnerability was disclosed to the developers by an outside source. Likewise, developers might or might not disclose the vulnerability publicly.

The fix event is the earliest date on which a user of software affected by a vulnerability could update their software system to a new release that is not affected by the vulnerability. Typically the fix to the vulnerability is disseminated in a new software release by the developer community.

The three time intervals in Figure 3 are (1) the time to discover a vulnerability $\left(t_{d}\right.$, specified as the time interval between introduction and discovery), (2) the time to fix a vulnerability ( $t_{f}$, specified as the time interval between discovery and fix), and (3) the time of exposure ( $t_{e}$, specified as the time interval between introduction and fix; also the sum of $t_{d}$ and $t_{f}$ ). Each time interval is the time between two events.

The vulnerability lifecycle model in Figure 3 provides a conceptual framework for clear thinking about software vulnerabilities and secure software. According to the model, the following actions will result in improved software with fewer vulnerabilities: (1) introduce fewer vulnerabilities, (2) discover vulnerabilities more quickly by shortening the time to discover $\left(t_{d}\right)$, (3) fix vulnerabilities more quickly by shortening the time to fix $\left(t_{f}\right)$. Actions (1) and (2) both shorten the total time of exposure $\left(t_{e}\right)$. Likewise, the following actions will result in improved software with less-severe vulnerabilities: (4) introduce vulnerabilities of lower severity, and (5) prioritize fixing of higher severity vulnerabilities first. Different activities may impact different actions.

The next section demonstrates the use of the vulnerability lifecycle model to examine software vulnerabilities in the open source Apache HTTP Server project. 


\subsection{Application case: The Apache HTTP Server Project}

The Apache HTTP Server project has a strong reputation in the open source community for attention to security. The security reports at the project website systematically report the vulnerabilities fixed in each release. For example, the security report for release 2.4 (the current major release as of July 2015) stated the following:

This page lists all security vulnerabilities fixed in released versions of Apache httpd 2.4. Each vulnerability is given a security impact rating by the Apache security team - please note that this rating may well vary from platform to platform. We also list the versions of Apache httpd the flaw is known to affect, and where a flaw has not been verified list the version with a question mark.

Please note that if a vulnerability is shown below as being fixed in a "-dev" release then this means that a fix has been applied to the development source tree and will be part of an upcoming full release.

This page is created from a database of vulnerabilities originally populated by Apache Week. Please send comments or corrections for these vulnerabilities to the Security Team.

The initial GA release, Apache httpd 2.4.1, includes fixes for all vulnerabilities which have been resolved in Apache httpd 2.2.22 and all older releases. Consult the Apache httpd 2.2 vulnerabilities list for more information.

The description of each vulnerability includes the release in which the vulnerability was fixed, the CVE ID, an impact rating (low, moderate, or important) assigned by the Apache security team, a description written by the security team, an acknowledgements of the contributor who reported the issue, the date that the vulnerability was reported to the security team, the date that the issue was made public, the date that an update was released, and a list of the release numbers of the versions affected by the vulnerability.

Table 8 compiles the vulnerability information for the 23 vulnerabilities fixed in the ten releases 2.4 up to release 2.4.16 (July 15 2015). Figure 4 is a visual timeline. 
Table 8: Vulnerabilities fixed in Apache HTTP Server release 2.4.x

\begin{tabular}{|c|c|c|c|c|c|c|c|c|c|c|c|c|c|c|}
\hline & CVE ID & \multicolumn{3}{|c|}{ Dates } & \multicolumn{10}{|c|}{ Affected } \\
\hline 1 & CVE-2012-0883 & $2012-02-14$ & 2012-03-02 & $2012-04-17$ & $\bullet$ & & & & & & & & & \\
\hline 2 & CVE-2012-2687 & 2012-05-31 & 2012-06-13 & 2012-08-21 & $\bullet$ & $\bullet$ & & & & & & & & \\
\hline 3 & CVE-2012-3502 & & 2012-08-16 & 2012-08-21 & $\bullet$ & $\bullet$ & & & & & & & & \\
\hline 6 & CVE-2013-2249 & 2013-05-29 & $2013-07-22$ & 2013-07-22 & $\bullet$ & $\bullet$ & $\bullet$ & $\bullet$ & & & & & & \\
\hline 7 & CVE-2013-1896 & 2013-03-07 & $2013-05-23$ & $2013-07-22$ & $\bullet$ & $\bullet$ & $\bullet$ & $\bullet$ & & & & & & \\
\hline 8 & CVE-2013-6438 & $2013-12-10$ & 2014-03-17 & 2014-03-17 & $\bullet$ & $\bullet$ & $\bullet$ & $\bullet$ & $\bullet$ & $\bullet$ & & & & \\
\hline 9 & CVE-2014-0098 & $2014-02-25$ & 2014-03-17 & 2014-03-17 & $\bullet$ & • & $\bullet$ & $\bullet$ & $\bullet$ & $\bullet$ & & & & \\
\hline 10 & CVE-2013-4352 & 2013-09-14 & 2014-07-14 & 2013-11-26 & & & & & $\bullet$ & & & & & \\
\hline 11 & CVE-2014-0226 & 2014-05-30 & 2014-07-14 & 2014-07-14 & $\bullet$ & $\bullet$ & $\bullet$ & $\bullet$ & $\bullet$ & $\bullet$ & $\bullet$ & & & \\
\hline 15 & CVE-2014-0231 & $2014-06-16$ & $2014-07-14$ & 2014-07-14 & $\bullet$ & $\bullet$ & $\bullet$ & $\bullet$ & $\bullet$ & $\bullet$ & $\bullet$ & & & \\
\hline 16 & CVE-2013-5704 & 2013-09-06 & 2013-10-19 & $2015-01-30$ & $\bullet$ & $\bullet$ & $\bullet$ & $\bullet$ & $\bullet$ & $\bullet$ & $\bullet$ & $\bullet$ & & \\
\hline 17 & CVE-2014-3581 & & 2014-09-08 & $2015-01-30$ & $\bullet$ & $\bullet$ & $\bullet$ & $\bullet$ & $\bullet$ & $\bullet$ & $\bullet$ & $\bullet$ & & \\
\hline & CVE-2014-3583 & 2014-09-17 & 2014-11-12 & $2015-01-30$ & & & & & & & & $\bullet$ & & \\
\hline 19 & CVE-2014-8109 & & 2014-11-09 & $2015-01-30$ & $\bullet$ & $\bullet$ & $\bullet$ & $\bullet$ & $\bullet$ & $\bullet$ & $\bullet$ & $\bullet$ & & \\
\hline & CVE-2015-3185 & 2013-08-05 & 2015-06-09 & $2015-07-15$ & $\bullet$ & $\bullet$ & $\bullet$ & $\bullet$ & $\bullet$ & $\bullet$ & $\bullet$ & $\bullet$ & $\bullet$ & \\
\hline 21 & CVE-2015-3183 & 2015-04-04 & 2015-06-09 & $2015-07-15$ & $\bullet$ & $\bullet$ & $\bullet$ & $\bullet$ & $\bullet$ & $\bullet$ & $\bullet$ & $\bullet$ & $\bullet$ & \\
\hline 22 & CVE-2015-0253 & 2015-02-03 & 2015-03-05 & $2015-07-15$ & & & & & & & & & $\bullet$ & \\
\hline \multirow{2}{*}{\multicolumn{2}{|c|}{23 CVE-2015-0228 }} & $2015-01-28$ & 2015-02-04 & 2015-07-15 & & & & & $\bullet$ & $\bullet$ & $\bullet$ & $\bullet$ & $\bullet$ & \\
\hline & & & & & 18 & 17 & 15 & 13 & 14 & 13 & 11 & 7 & 4 & 0 \\
\hline
\end{tabular}




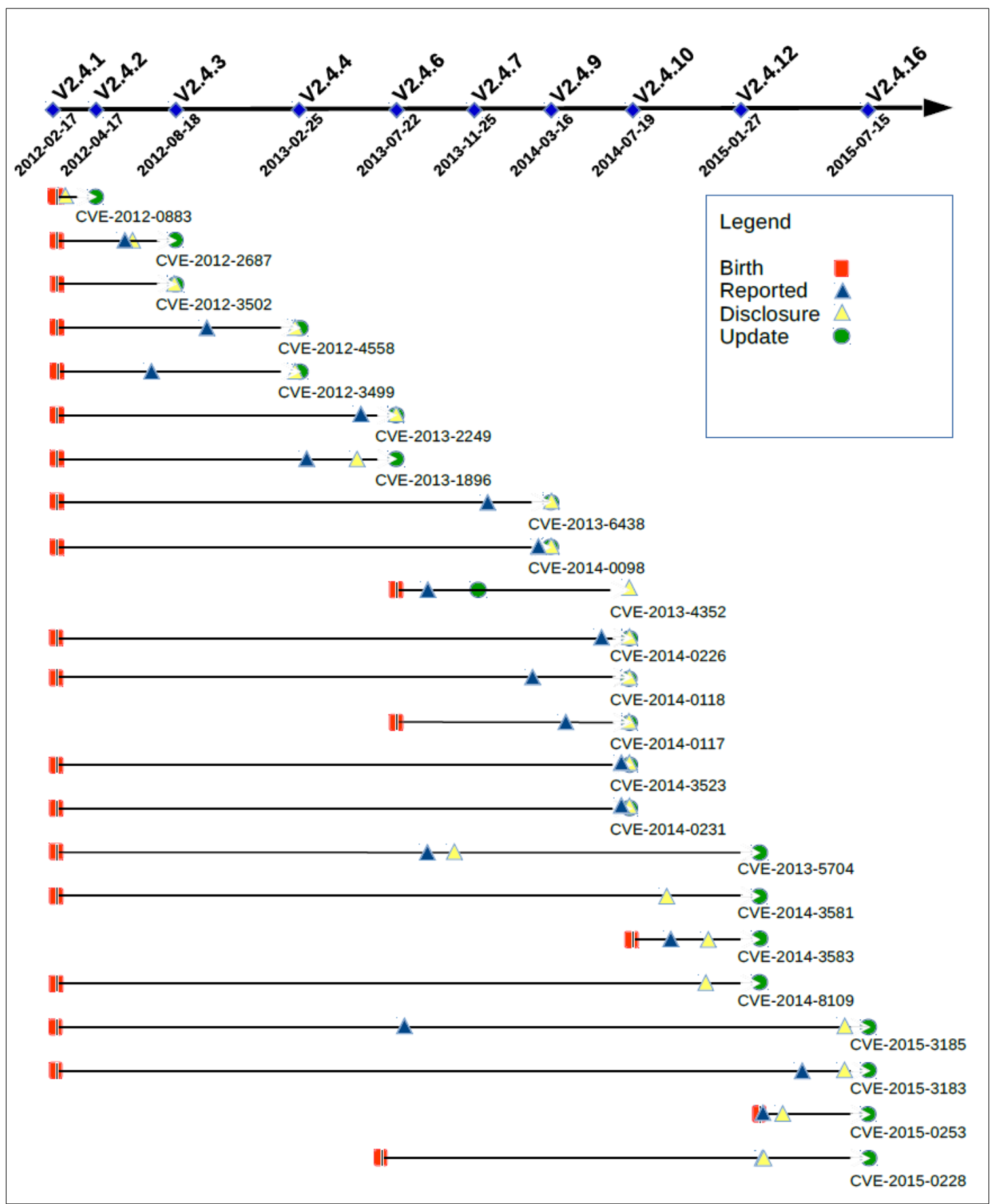

Figure 4: Timeline of vulnerabilities fixed in Apache HTTP Server 2.4.x 
Some observations from this exercise:

1) Vulnerabilities introduced in one release are fixed in later a release.

2) Vulnerabilities are discovered and reported by individual people; the Apache project acknowledges these contributions and identifies the contributor by name.

3) Newly-discovered vulnerabilities often affect previous releases. For example, CVE2015-3183, fixed in release 2.4.16 (July 152015 , same as the "update released" date), was publicly disclosed on June 92015 (the "Issue public" date) after being reported to the project two months earlier on April 42015 (the "Reported to security team" date), but affected releases back to 2.4.1 (released February 17 2012) and perhaps earlier.

4) Public disclosure typically happens after the vulnerability was reported to the project. Public disclosure is sometimes delayed until the release when the vulnerability is fixed. 5) In this project, older releases contain more known vulnerabilities.

6) Newer releases can be affected by vulnerabilities not affecting older releases.

These results suggest that release dates, if the project release history and the releases affected by a vulnerability are known, could proxy for the introduction and fix events. An additional data source, such as the Apache "Reported to security team" field, would be needed to specify the discovery event.

This chapter has developed a conceptual framework for software vulnerabilities. The next chapter develops a set of testable hypothesis, bringing together assertions from the scholarly and practitioner literature reviewed in chapter 2 , the constructs from the conceptual frameworks developed in chapter 3 , and the information available in the publicly-available data sets reviewed in the chapter 4 . 


\section{Hypotheses}

This chapter develops the hypotheses to be tested. The set of twelve hypotheses relate seven constructs with three dependent response variables about software vulnerabilities (i.e., the time to discover a vulnerability, the time to fix a vulnerability, and the total time of exposure) and four independent explanatory variables about project and community attributes (related to project contributors, commits, comments, and code churn). Figure 5 provides a graphical representation.

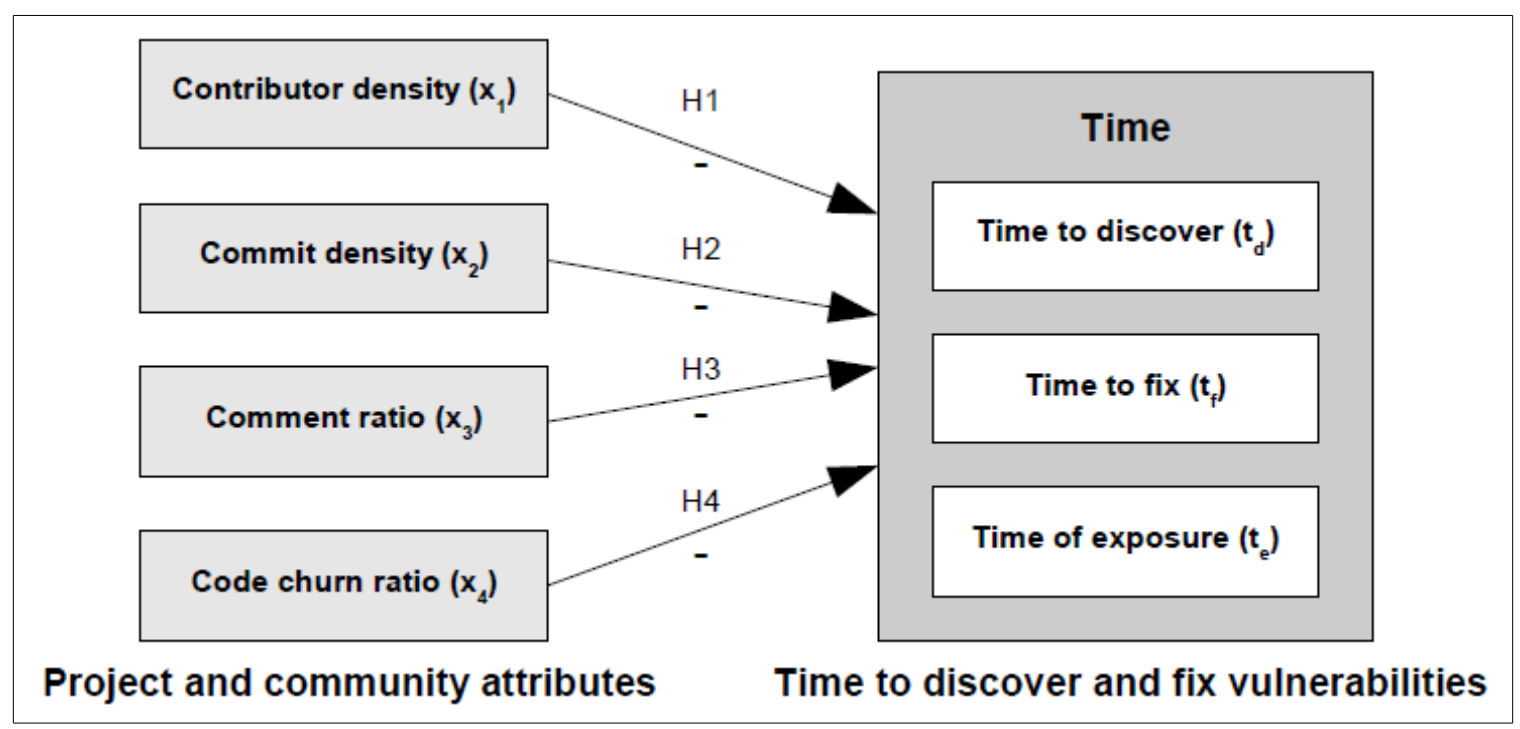

Figure 5: Hypotheses

These hypotheses are intended bring together the assertions in the scholarly and practitioner literature reviewed in chapter 2, with the availability of metrics about open source software projects and software vulnerabilities in the online databases examined in chapter 3, and the conceptual framework of the software vulnerability lifecycle developed in chapter 4. Each exists at the intersection of interesting assertions, data availability, and relevance to the objective and guiding research questions. 


\subsection{Contributors (H1)}

The potential for contribution is a defining feature of open source software. In a statement responding to the 2014 GNU Bash "Shellshock" vulnerability, the Free Software Foundation (2014) explained its position as follows:

When a bug is discovered in free software, everyone has permission, rights, and source code to expose and fix the problem. That fix can then be immediately freely distributed to everyone who needs it. Thus, these freedoms are crucial for ethical, secure computing.

A large and active development community is widely perceived as a measure of success for an open source software project (Fogel, 2006; Wheeler \& Khakimov, 2015) - a much sought-after outcome of doing things right (West \& O'Mahony, 2008; Muegge, 2011; Schweik, 2013). Projects with large and active communities of contributors not only benefit from the option of inspection, modification, and sharing (Sullivan, 2014), they experience the direct results of contributors - including finding and reporting previously undiscovered vulnerabilities. Raymond (1999) writes:

More users find more bugs because adding more users adds more different ways of stressing the program. This effect is amplified when the users are codevelopers. Each one approaches the task of bug characterization with a slightly different perceptual set and analytical toolkit, a different angle on the problem...

Conversely, Constantin (2014) speculates that vulnerabilities are more likely to occur and remain undiscovered in projects with too few developers.

These arguments lead to the following hypothesis:

\section{H1a: Higher contributor density is associated with shorter vulnerability discovery time.}

H1a speaks of "contributor density" rather than number of contributors because 
open source projects vary widely in size of the source code base, commonly measured in thousands of lines of code (kLOC). The notion of contributor density takes these differences into account in the simplest way, by dividing the number of contributors by the size of the code base.

Once a vulnerability has been discovered and is known to contributors, Linus's Law comes into play (Raymond, 1999): "Given a large enough beta-tester and codeveloper base, almost every problem will be characterized quickly and the fix obvious to someone. Or, less formally, 'Given enough eyeballs, all bugs are shallow." Thus:

H1b: Higher contributor density is associated with shorter vulnerability fixing time.

The arguments for $\mathrm{H} 1 \mathrm{a}$ and $\mathrm{H} 1 \mathrm{~b}$ also lead to the following hypothesis:

\section{H1c: Higher contributor density is associated with shorter exposure time.}

\subsection{Commit activity (H2)}

A commit to an open source project is a change to the source code. Thus commits is a measure of project activity - how much work is happening. A commit indicates that a contributor has engaged with the source code, and each engagement is another opportunity to discover vulnerabilities. Conversely, Wheeler \& Khakimov (2015) associate low project activity with "projects at risk" needing more outside investment. Perlroth (2014) speculates that the Heartbleed vulnerability remained undiscovered for so long because of low project activity. Stated formally:

\section{H2a: Higher commit density is associated with shorter vulnerability discovery time.}

H2a speaks of "density" rather than a simple count for the same reasons as H1ato take into account the differences between large and small projects in a simple way. 
High commit activity implies that contributors are actively engaged with the source code. After a vulnerability is discovered, an engaged community can rapidly respond. In the words of Jim Zemlin, Executive Director of the Linux Foundation (Kerner, 2015): "We all have access and can all work together.... Open source by its nature lets us have a collective response to a collective problem." High commit activity is an indication that a collective response can and is happening. Stated formally:

H2b: Higher commit density is associated with shorter vulnerability fixing time.

The arguments for $\mathrm{H} 2 \mathrm{a}$ and $\mathrm{H} 2 \mathrm{~b}$ also lead to the following hypothesis:

H2c: Higher commit density is associated with shorter vulnerability exposure time.

\subsection{Comments (H3)}

Complex code is more difficult to understand, maintain, and test (McCabe, 1976; McGraw, 2006; Shin et al. 2011). According to Schneier (2003), complexity is the enemy of security. Comments are a way to tame complexity - to make code easier to understand. Conversely, software with few comments may be harder to review, and thus vulnerabilities may be easier to miss (Wheeler \& Khakimov, 2015). Comment ratio is the proportion of comments in the source code base. Thus:

H3a: Higher comment ratio is associated with shorter vulnerability discovery time.

Once a vulnerability has been discovered, well-commented code will be easier update; learning can happen more quickly. Stated formally:

H3b: Higher comment ratio is associated with shorter vulnerabilities fixing time.

The arguments for $\mathrm{H} 3 \mathrm{a}$ and $\mathrm{H} 3 \mathrm{~b}$ also lead to the following hypothesis:

H3c: Higher comment ratio is associated with shorter exposure time. 


\subsection{Code churn (H4)}

Shin et al. (2011, p. 774) writes: "Code is constantly evolving throughout the development process. Each new change in the system brings a new risk of introducing a vulnerability." Previous studies of vulnerability prediction have associated high code churn metrics with introduction of new vulnerabilities (Gegick et al. 2008; Meneely et al. 2013). However, the interest here is not vulnerability introduction but rather the time to discover and fix vulnerabilities already affecting the software. Code churn ratio defined here as the proportion of the code base that has changed - is a measure of turnover in the code base. As argued previously, engagement with the source code creates opportunities to discover vulnerabilities. Stated formally:

\section{H4a: Higher code churn ratio is associated with shorter vulnerability discovery time.}

Very low levels of code churn ratio imply low project activity. Some turn-over is expected in an active project that is more likely to fix vulnerabilities promptly after they are discovered. Stated formally:

\section{H4b: Higher code churn ratio is associated with shorter vulnerability fix time.}

The arguments for $\mathrm{H} 4 \mathrm{a}$ and $\mathrm{H} 4 \mathrm{~b}$ also lead to the following hypothesis:

\section{H4c: More code churn density is associated with shorter exposure time.}

The chapter has developed a set of twelve testable hypotheses in four groups, relating four explanatory variables about open source software projects to three response variables about the time to discover and fix vulnerabilities. The next chapter specifies the research design and method used to test these hypotheses. 
An investigation of software vulnerabilities in open source software projects using data from publicly-available sources

Copyright (C) 2017 S. M. Monzur Murshed

\section{Research design and method}

This chapter presents the research design and detailed method to answer the research questions posed in chapter 1 . Table 9 summarizes nine-step research method, the activity undertaken at each step, and the outcomes of each activity. The remainder of this chapter is structured in nine sections, one for each step of the research method summarized in Table 9.

Table 9: Nine steps of the research method

\begin{tabular}{|c|c|c|}
\hline Step & Activity undertaken & Outcomes of activity \\
\hline $\begin{array}{l}\text { 1. Identify } \\
\text { assertions }\end{array}$ & $\begin{array}{l}\text { Examine the scholarly and practitioner literature to } \\
\text { identify assertions about security vulnerabilities in } \\
\text { open source software }\end{array}$ & $\begin{array}{l}\text { - Set of assertions } \\
\text { (section 2.4) }\end{array}$ \\
\hline $\begin{array}{l}\text { 2. Investigate data } \\
\text { sets }\end{array}$ & $\begin{array}{l}\text { Examine the content of two websites: Black Duck } \\
\text { Open Hub and CVE Details }\end{array}$ & $\begin{array}{l}\text { - List of available metrics } \\
\text { (chapter } 3 \text { ) }\end{array}$ \\
\hline $\begin{array}{l}\text { 3. Develop } \\
\text { conceptual } \\
\text { framework }\end{array}$ & Develop a vulnerability lifecycle model & $\begin{array}{l}\text { - Vulnerability life-cycle model } \\
\text { (chapter 4) }\end{array}$ \\
\hline $\begin{array}{l}\text { 4. Develop } \\
\text { hypotheses }\end{array}$ & $\begin{array}{l}\text { Develop a set of hypotheses at the intersection of } \\
\text { (i) assertions in the research literature and } \\
\text { practitioner literature, (ii) data available from publicly- } \\
\text { available sources, and (iii) events in the conceptual } \\
\text { framework }\end{array}$ & $\begin{array}{l}\text { - Set of hypotheses } \\
\text { (chapter 5) }\end{array}$ \\
\hline 5. Select samples & $\begin{array}{l}\text { Establish processes to select projects for the } \\
\text { research samples }\end{array}$ & $\begin{array}{l}\text { - Rules for sample selection } \\
\text { (section 6.5) } \\
\text { - Sample of open source } \\
\text { software projects } \\
\text { (section 7.1) }\end{array}$ \\
\hline $\begin{array}{l}\text { 6. Inspect small } \\
\text { sample of CVEs }\end{array}$ & $\begin{array}{l}\text { Manually examine the database records of a small } \\
\text { sample of CVEs. }\end{array}$ & $\begin{array}{l}\text { - Insights into data availability } \\
\text { and data quality } \\
\text { (section } 7.2 \text { ) }\end{array}$ \\
\hline $\begin{array}{l}\text { 7. Specify variables } \\
\text { and measures for } \\
\text { full sample }\end{array}$ & $\begin{array}{l}\text { Identify operational variables, measures for each } \\
\text { variable, sources for each measure, and tests of } \\
\text { each hypothesis }\end{array}$ & $\begin{array}{l}\text { - Specification of dependent and } \\
\text { independent variables and } \\
\text { tests of hypotheses } \\
\text { (section 6.7) }\end{array}$ \\
\hline $\begin{array}{l}\text { 8. Collect data for } \\
\text { full sample }\end{array}$ & $\begin{array}{l}\text { Develop software scripts to harvest data from } \\
\text { publicly-available websites. Run the scripts. }\end{array}$ & $\begin{array}{l}\text { - Workflow and scripts } \\
\text { (Appendix A and B) } \\
\text { - R data frame (CSV file) }\end{array}$ \\
\hline $\begin{array}{l}\text { 9. Analyze full } \\
\text { sample }\end{array}$ & $\begin{array}{l}\text { Compute summary statistics. Inspect variables. } \\
\text { Compute regressions. Interpret results. }\end{array}$ & $\begin{array}{l}\text { - Results of regression analysis } \\
\text { and hypothesis tests } \\
\text { (section 7.3) }\end{array}$ \\
\hline
\end{tabular}


The research design is quantitative hypothesis testing of associations between variables. The unit of analysis is a vulnerability in the software produced by an open source software project, operationalized as a CVE ID. The data set is constructed from publicly-available online sources using automated data collection techniques (Munzert et al. 2015). The analytic approach is linear regression analysis using the $\mathrm{R}$ language for statistical computing (R Core Team, 2016) and the techniques recommended by Fox (2016) and Fox \& Weisberg (2011).

\subsection{Identify assertions from the literature}

The assertions found in the academic research literature and the practitioner literature were previously reported in section 2.4 .

\subsection{Investigate data sets}

The various metrics reported by CVE Details and Black Duck Open Hub were previously reported in sections 3.1 and 3.2, respectively.

\subsection{Develop conceptual framework}

The software vulnerability lifecycle model was previously presented in section 4.1, and application of the lifecycle model was demonstrated in section 4.2 by characterizing a limited set of 23 software vulnerabilities fixed during release 2.4 of the Apache HTTP Server project.

The software vulnerability lifecycle model is comprised of three events and three time intervals. The three events are (1) introduction of the vulnerability, (2) discovery of the vulnerability, and (3) dissemination of a fix to the vulnerability. Each event is specified by the date on which the event occurred. The three time intervals are (1) the 
time to discover a vulnerability $\left(t_{d}\right.$, specified as the time interval between introduction and discovery), (2) the time to fix a vulnerability ( $t_{f}$, specified as the time interval between discovery and fix), and (3) the time of exposure ( $t_{e}$, specified as the time interval between introduction and fix; also the sum of $t_{d}$ and $t_{f}$ ). Each time interval is the time between two events.

\subsection{Develop hypotheses}

The hypotheses were previously developed in chapter 5. Each hypothesis is intended to formalize an assertion from the literature (identified in step 1, sections 2.4 and 6.1), specified using a conceptual framework of the software vulnerability lifecycle (developed in step 3, sections 4.1 and 6.3), for which data is available (confirmed in step 2, sections $3.1,3.2$ and 6.2).

\subsection{Select samples}

This thesis employs three nested research samples: (1) the project sample of open source software projects, (2) a small sample of 10 software vulnerabilities within the project sample, used for manual inspection in step 6, and (3) the full sample of all vulnerabilities within the project sample, used for statistical analysis and hypothesis testing in step 8 .

The project sample was selected using a three-step process. First, we limited our scope to open source content management system (CMS) projects. This category has several attractive features: it encompasses a large number of open source projects that vary widely in their properties (e.g., in January 2016, there were more than one hundred open source CMS projects with Wikipedia articles), it features prominently in 
publications by security researchers (e.g., Walden, 2009, 2010; Vasek et al. 2016) and in industry reports of threats and breaches such as the Verizon 2016 Data Breach Investigations Report (Verizon, 2016) and the Symantec 2016 Internet Security Threat Report (Symantec Corporation, 2016), it is central to the enterprise systems of many organizations, and there are various inventories and lists of projects available online.

Second, we compiled a list of 290 candidate projects from sources about open source CMS projects including Open Source CMS (http://www.opensourcecms.com/), Securify Graphs (http://www.securifylabs.com/securifygraphs/), Bitnami (https://bitnami.com/), and the Wikipedia list of content management systems (https://en.wikipedia.org/wiki/List_of_content_management_systems).

Third, we considered data availability using three criteria: (i) affected by at least one CVE in CVE Details, (ii) project history available from Open Hub, and (iii) information on release history and dates of relevant releases is readily available online. The third criterion is needed to assign a proxy date for the introduction event and fix event for a CVE. After eliminating those projects that were unhelpful for testing our hypotheses, sixty projects remained. The project sample is reported in section 7.1.

Next, we selected a small sample of vulnerabilities within the project sample for manual inspection of the CVE records. We began by compiling a list of CVEs affecting the projects in the project sample, then selected ten CVEs at random from the list. The small sample is reported in section 7.2.

The full sample is the complete inventory of vulnerabilities affecting the projects in the project sample. 


\subsection{Inspect small sample}

For each of the ten vulnerabilities in the small sample, we manually examined the content of each CVE record, seeking insights to help specify variables and measures in step 7 and to inform the automated data collection of the full sample in step 8 . There were four guiding questions: (1) Can "Publish Date" or "Update Date" from the CVE Details summary table reports be used as proxies for the events in the vulnerability life cycle model? (2) Can the versions numbers reported in the "Products Affected by CVE" category of the CVE Details individual CVE records be used as proxies for the events in the vulnerability life cycle model? (3) What can be learned from the linked documents reported in the "References for CVE" category of the CVE Details individual CVE records? (4) Can information from the references identify the events in the vulnerability lifecycle model?

Results of manually inspecting the small sample of vulnerabilities are reported in section 7.2. Insights from these results are enfolded into steps 7, 8, and 9 of the method.

\subsection{Specify variables and measures for full sample}

Table 10 is a summary of the variables, measures, and sources. 
Table 10: Variables, measures, and sources for full sample analysis

\begin{tabular}{|c|c|c|c|c|}
\hline Type & Variable & Definition & Measure & Source \\
\hline \multirow{8}{*}{$\begin{array}{l}\text { Explanatory } \\
\text { variables }\end{array}$} & \multirow{2}{*}{$\begin{array}{l}\text { Contributor } \\
\text { density } \\
\left(x_{1}\right)\end{array}$} & \multirow{2}{*}{$\begin{array}{l}\text { The ratio of the number of contributors } \\
\text { to the size of the code base (thousand } \\
\text { lines of code); a measure of community } \\
\text { participation }\end{array}$} & \# of contributors & \multirow{8}{*}{ Open Hub } \\
\hline & & & $\begin{array}{l}\text { kilo lines of code } \\
(\mathrm{kLOC})\end{array}$ & \\
\hline & \multirow{2}{*}{$\begin{array}{l}\text { Commit } \\
\text { density } \\
\left(x_{2}\right)\end{array}$} & \multirow{2}{*}{$\begin{array}{l}\text { The ratio of the number of monthly } \\
\text { commits to the size of the code base } \\
\text { (thousand lines of code); a measure of } \\
\text { developer activity }\end{array}$} & \# of contributors & \\
\hline & & & $\mathrm{kLOC}$ & \\
\hline & \multirow{2}{*}{$\begin{array}{l}\text { Comment } \\
\text { ratio } \\
\left(x_{3}\right)\end{array}$} & \multirow{2}{*}{$\begin{array}{l}\text { The ratio of comments to lines of code; } \\
\text { an approach to managing complexity }\end{array}$} & \# of comment LOC & \\
\hline & & & LOC & \\
\hline & \multirow{2}{*}{$\begin{array}{l}\text { Code churn } \\
\text { ratio } \\
\left(x_{4}\right)\end{array}$} & \multirow{2}{*}{$\begin{array}{l}\text { The ratio of the number of lines of code } \\
\text { modified to the size of the code base; a } \\
\text { measure of code base churn }\end{array}$} & \# of modified LOC & \\
\hline & & & $\mathrm{kLOC}$ & \\
\hline \multirow[t]{3}{*}{$\begin{array}{l}\text { Response } \\
\text { variables }\end{array}$} & $\begin{array}{l}\text { Time to } \\
\text { discover } \\
\left(t_{d}\right)\end{array}$ & $\begin{array}{l}\text { The time (number of days) between the } \\
\text { introduction event and the discovery } \\
\text { event of a CVE ID }\end{array}$ & $\mathrm{N} / \mathrm{a}$ & \multirow{3}{*}{$\begin{array}{l}\text { CVE } \\
\text { Details }\end{array}$} \\
\hline & $\begin{array}{l}\text { Time to fix } \\
\left(t_{f}\right)\end{array}$ & $\begin{array}{l}\text { The time (number of days) between the } \\
\text { discovery event and the fix event of a } \\
\text { CVE ID }\end{array}$ & $\mathrm{N} / \mathrm{a}$ & \\
\hline & $\begin{array}{l}\text { Time of } \\
\text { exposure } \\
\left(t_{e}\right)\end{array}$ & $\begin{array}{l}\text { The time (number of days) between the } \\
\text { introduction event and the fix event of a } \\
\text { CVE ID }\end{array}$ & $\begin{array}{l}\text { (Release date of first } \\
\text { unaffected version) - } \\
\text { (Release date of first } \\
\text { affected version) }\end{array}$ & \\
\hline
\end{tabular}

Comment ratio $\left(x_{3}\right)$, measuring one approach to managing complexity, is obtained directly from Open Hub - the ratio of comment lines to total lines of code in the month of the focal event. The other three explanatory variables are computed as quotients of two metrics obtained from Open Hub, the denominator for each being the size of the code base (in kLOC). Contributor density $\left(x_{l}\right)$, a measure of community participation, is the ratio of the number of contributors in the month of the focal event to the size of the code base in the month of the focal event. Commit density $\left(x_{2}\right)$, a measure of developer activity, is the ratio of the number of commits in the month of the focal event to the size of the code base in the month of the focal event. Code churn ratio $\left(x_{4}\right)$ is the ratio of the 
number of lines of code modified in the month of the focal event to the size of the code base in the month of the focal event.

The focal event is the terminal event for response variable of interest (q.v., Figure 3 of the vulnerability life cycle model in section 4.1). For time to discover $\left(t_{d}\right)$, the focal event is the discovery event; thus the explanatory variables are computed using project metrics from the month in which the vulnerability was discovered. For time to fix $\left(t_{f}\right)$ and time of exposure $\left(t_{e}\right)$, the focal event is the fix event; thus the explanatory variables are computed using project metrics from the month in which the vulnerability was fixed.

The outcome of step 6, manual inspection of a small sample of vulnerabilities, demonstrated a significant limitation in the publicly-available data sets: the lack of a strong proxy for the discovery event, the second of the three events in the vulnerability life-cycle framework previously developed in section 4.1. Two of the three intended response variables, time to discover $\left(t_{d}\right)$ and time to fix $\left(t_{f}\right)$, require an estimate of the discovery event. Thus, Table 10 shows that only the third response variable, time of exposure $\left(t_{e}\right)$, is sufficiently specified for data analysis in step 9 . The value assigned to time of exposure $\left(t_{e}\right)$ is the number of days between the release of the first affected version (a proxy for the introduction event) and the release of the first unaffected version after introduction of the vulnerability (a proxy for the fix event). The focal event is thus the fix event (i.e., the month of release of the first unaffected version after introduction of the vulnerability), and the explanatory variables for a particular vulnerability are assigned using project metrics for the month of the fix event. 


\subsection{Collect data for full sample}

Appendix A specifies the data collection workflow, documents the operation of each script, and provides instructions for modifying the workflow and scripts for future work. Appendix B provides source code for the various R and Python scripts.

A brief summary is provided here:

1. An R script forms HTTP requests to CVE Details for summary table reports for each project, for each year, for all years of the project. CVE Details returns an HTML file for each request. The script outputs these files to a local directory.

2. A second R script parses the HTML files, creates an R data frame, and outputs a comma-separated values (CSV) file for each project.

3. The researcher manually inputs two data fields for each CVE record: (1) the release date of the first affected version (as a proxy for the introduction event), and (2) the release date of the first unaffected version after the introduction of the vulnerability (as a proxy for the fix event). These dates are discovered from online sources about the project release history.

4. A third $\mathrm{R}$ script joins the files for each project into a single $\mathrm{R}$ data frame, and outputs a CSV file.

5. A Python script forms HTTP GET requests using the Open Hub API for the ActivityFact and SizeFact records for each project. Open Hub returns XML responses for each request. The script outputs the ActivityFact.xml and SizeFact.xml files for each project to a local directory.

6. A fourth R script parses the XML files for each project and outputs a single CSV 
file for all projects. This file contains a complete project history, by month, for all projects in the project sample.

7. A fifth R script "merges" the file of vulnerability data and the file of project data. The explanatory variables are computed from project metrics in the month of the fix event. The script also computes time-averaged explanatory variables based on 3 months, 6 months, and 12 months of project metrics to smooth out month-tomonth variation in the project metrics. The script outputs a CSV file conveniently formatted for regression analysis.

Developing and testing the workflow and scripts for automated data collection was a significant effort for the author (see also section 8.2). Nonetheless, the most timeintensive data collection task was the manual work to identify and record the release dates of the first affected version and the first fixed version (step 3 of the data collection workflow in Appendix A). Insights from this experience informed the answers provided in section 8.1 to the second and third research questions.

\subsection{Statistical analysis of full sample}

Table 11 summarizes the nine steps of the full sample data analysis, following the approach and techniques recommended by Fox (2016) and Fox \& Weisberg (2011). Section 7.3 reports the results of this analysis. 
Table 11: Nine steps of full sample data analysis

\begin{tabular}{|c|c|c|}
\hline Step & Description & Outcomes \\
\hline $\begin{array}{l}\text { i. Examine } \\
\text { univariate data }\end{array}$ & $\begin{array}{l}\text { Inspect the data set. Plot histograms with boxplots of } \\
\text { each variable. }\end{array}$ & $\begin{array}{l}\text { - Histograms } \\
\text { - Boxplots }\end{array}$ \\
\hline $\begin{array}{l}\text { ii. Examine } \\
\text { bivariate } \\
\text { relationships }\end{array}$ & $\begin{array}{l}\text { Examine the relationships between variables. Plot a } \\
\text { scatterplot matrix with scatterplots of each variable pair. }\end{array}$ & - Scatterplot matrix \\
\hline $\begin{array}{l}\text { iii. Compute } \\
\text { summary } \\
\text { statistics }\end{array}$ & $\begin{array}{l}\text { Produce a table reporting the minimum value, maximum } \\
\text { value, range, mean, median, and standard deviation of } \\
\text { each variable and the correlation matrix. }\end{array}$ & - Table of summary statistics \\
\hline $\begin{array}{l}\text { iv. Identify and } \\
\text { assess } \\
\text { violations }\end{array}$ & $\begin{array}{l}\text { Identify and address violations of the regression } \\
\text { assumptions. Comment on multicollinearity and outliers. } \\
\text { Comment on whether action is required to address any } \\
\text { violations. Comment on any evidence of a linear } \\
\text { relationship (or nonlinear relationship) between the } \\
\text { dependent and independent variables. }\end{array}$ & - Possible violations \\
\hline $\begin{array}{l}\text { v. Regression } \\
\text { analysis }\end{array}$ & $\begin{array}{l}\text { Regress the response variables on the explanatory } \\
\text { variables. }\end{array}$ & $\begin{array}{l}\text { - Regression results: regression } \\
\text { coefficients, } \mathrm{R}^{2} \text {, and p-values }\end{array}$ \\
\hline $\begin{array}{l}\text { vi. Hypothesis } \\
\text { tests }\end{array}$ & $\begin{array}{l}\text { Report the results of the hypothesis tests. A hypothesis } \\
\text { is supported if and only if (i) the p-value is below a } \\
\text { critical threshold and (ii) the regression coefficient of the } \\
\text { independent variable has the predicted sign. }\end{array}$ & - Results of hypothesis tests. \\
\hline $\begin{array}{l}\text { vii. Post- } \\
\text { regression } \\
\text { diagnostics }\end{array}$ & Plot and examine residuals and unusual data. & $\begin{array}{l}\text { - Residual plots } \\
\text { - Influence plots }\end{array}$ \\
\hline $\begin{array}{l}\text { viii. Explore } \\
\text { robustness }\end{array}$ & $\begin{array}{l}\text { Explore the robustness of the regression model and the } \\
\text { hypothesis tests to modelling assumptions. }\end{array}$ & - Results of alternative models \\
\hline $\begin{array}{l}\text { ix. Examine } \\
\text { effect size }\end{array}$ & $\begin{array}{l}\text { Report the effect size and practical significance of the } \\
\text { regression results. }\end{array}$ & - Effect size plots \\
\hline
\end{tabular}

The chapter has specified the research design and method of this thesis. The next chapter presents the results. 
An investigation of software vulnerabilities in open source software projects using data from publicly-available sources

Copyright (C) 2017 S. M. Monzur Murshed

\section{Results}

This chapter presents the results in three sections: description of the sample of open source software projects, manual inspection of a small sample of vulnerabilities, and statistical analysis of the full sample of software vulnerabilities.

\subsection{Sample of open source software projects}

Table 12, Table 13, and Table 14 provide a summary of the sixty projects in the project sample, with their CPE product names (the label used as a unique identifier in CVE records), a brief description (using "tagline" words from the project website), the URL for the project website, the year that the project started, the size of the code base (in $\mathrm{kLOC}$ - thousands of lines of source code), the open source license(s) used, and the number of CVEs affecting the project's software reported by CVE Details. The method for selecting the project sample was previously explained in section 6.5. 
Table 12: Summary of project sample ( $n=60$; part 1 of 3$)$

\begin{tabular}{|c|c|c|c|c|c|c|c|}
\hline Project & $\begin{array}{l}\text { CPE product name } \\
\text { (CVE record) }\end{array}$ & Description & Website & $\begin{array}{l}\text { Year } \\
\text { started }\end{array}$ & $\begin{array}{l}\text { Code base } \\
\text { (kLOC) }\end{array}$ & Licenses & \# of CVEs \\
\hline ActionApps & actionapps & Collaborative web publishing tool & http://actionapps.org/ & 2000 & 202 & GPL & 1 \\
\hline Anchor CMS & anchorcms & Super-simple, lightweight blog system & https://anchorcms.com/ & 2011 & 16 & GPL v3 & 3 \\
\hline b2evolution & b2evolution & Complete engine for website & http://b2evolution.net/ & 2003 & 686 & GPL v2+ & 12 \\
\hline BEdita & bedita & Semantic content management framework & http://www.bedita.com/ & 2006 & 61 & LGPL & 2 \\
\hline BigTree CMS & bigtreecms & Extensible CMS built on PHP and MySQL & https://bigtreecms.org/ & 2012 & 251 & GPL v3 & 4 \\
\hline bitweaver & bitweaver & Web application framework & http://www.bitweaver.org/ & 2005 & 442 & LGPL & 21 \\
\hline CMSMadeSimple & cmsmadesimplecms & Power for professionals simplicity for users & https://www.cmsmadesimple.org/ & 2004 & 230 & GPL v2+ & 31 \\
\hline concrete5 & concrete5 & Provides version management for pages & https://www.concrete5.org/ & 2008 & 279 & MIT & 6 \\
\hline Croogo & croogo & Open source CMS built for everyone & https://croogo.org/ & 2009 & 52 & MIT & 2 \\
\hline DokuWiki & dokuwiki & Highly versatile wiki, no database required & https://www.dokuwiki.org/ & 2005 & 206 & GPL v2 & 29 \\
\hline dotclear & dotclear & Blogging platform in $\mathrm{PHP}$ & https://dotclear.org/ & 2011 & 394 & GPL v2 & 20 \\
\hline Drupal & drupal & Platform to publish, manage a website & https://www.drupal.org/ & 2000 & 704 & GPL v2+ & 162 \\
\hline e107 & e107 & Bootstrap & http://e107.org/ & 2004 & 525 & GPL & 60 \\
\hline Share.ez.no & ezpublish & Enterprise content management system & http://share.ez.no/ & 2002 & 2785 & GPL & 19 \\
\hline Gallery & gallery & Web based photo album organizer & http://galleryproject.org/ & 2002 & 311 & GPL v2+ & 58 \\
\hline Geeklog & geeklog & Spam protected CMS or blog engine & https://www.geeklog.net/ & 2001 & 649 & GPL v2+ & 27 \\
\hline Habari & habari & Blogging platform for blog or web journal & http://habariproject.org/ & 2006 & 48 & Apache 2 & 2 \\
\hline impresscms & impresscms & Tools for building, maintaining website & http://www.impresscms.org/ & 2007 & 245 & GPL v2+ & 9 \\
\hline IMPRESSPAGES & impresspages & MVC engine based on PHP components & https://www.impresspages.org/ & 2007 & 138 & MIT & 1 \\
\hline Jaws & jaws & Framework to hack own modules & http://jaws-project.com/ & 2005 & 675 & GPL v2+ & 9 \\
\hline
\end{tabular}


Table 13: Summary of project sample ( $n=60$; part 2 of 3$)$

\begin{tabular}{|c|c|c|c|c|c|c|c|}
\hline Project & $\begin{array}{l}\text { CPE product } \\
\text { (CVE record) }\end{array}$ & Description & Website & $\begin{array}{l}\text { Year } \\
\text { started }\end{array}$ & $\begin{array}{l}\text { Code base } \\
\text { (kLOC) }\end{array}$ & Licenses & \# of CVEs \\
\hline Joomla! & joomla & The CMS millions of websites are built on & https://www.joomla.org/ & 2005 & 355 & GPL & 176 \\
\hline Kajona & kajona & Content management framework & https://www.kajona.de & 2007 & 26 & LGPL & 4 \\
\hline LifeType & lifetype & Platform support multiple blog and users & http://lifetype.net/ & 2004 & 157 & GPL v2+ & 10 \\
\hline Magento & magento & Cloud analytics for commerce & https://magento.com/ & 2008 & 8259 & OSL v3 & 6 \\
\hline mahaRa & mahara & App for journals, social media resources & https://mahara.org/ & 2006 & 507 & GPL v3 & 39 \\
\hline MediaWiki & mediawiki & Wiki originally for use on Wikipedia & https://www.mediawiki.org & 2003 & 494 & GPL v2+ & 133 \\
\hline MONSTRA & monstra & Lightweight content management system & http://monstra.org/ & 2011 & 110 & GPL v3 & 1 \\
\hline Moodle & moodle & For personalised learning environments & https://moodle.org/ & 2001 & 3098 & GPL v3+ & 318 \\
\hline MOVABLETYPE & movabletype & Popular blog publishing platform & https://movabletype.org/ & 2006 & 769 & GPL v2+ & 32 \\
\hline nibbleblog & nibbleblog & Powerful engine for creating blogs & http://www.nibbleblog.com/ & 2012 & 25 & GPL v3 & 3 \\
\hline ocPortal & ocportal & Web 2.0 features support galleries, news & http://ocportal.com/start.htm & 2011 & 806 & CPAL v1 & 5 \\
\hline omeka & omeka & $\begin{array}{l}\text { Platform for publishing collection, } \\
\text { exhibition }\end{array}$ & https://omeka.org/ & 2006 & 73 & GPL v3+ & 1 \\
\hline OpenCms & opencms & Sophisticated engine W3C standard & http://www.opencms.org/en/ & 2002 & 2796 & GPL & 14 \\
\hline oscommerce & oscommerce & Online shop e-commerce solution & https://www.oscommerce.com/ & 2000 & 211 & GPL v2+ & 29 \\
\hline Ovidentia & ovidentia & Integrate publishing content on the web & http://www.ovidentia.org/ & 2001 & 222 & GPL v2+ & 5 \\
\hline phpBB & phpbb & Bulletin board software for security & https://subcompact/ & 2001 & 737 & GPL v2+ & 103 \\
\hline implore & implore & Information management framework & https://www.pimcore.org/en & 2011 & 257 & GPL v3+ & 4 \\
\hline Pivot & pivot & Tools for dynamics site like online journals & http://pivotlog.net/ & 2003 & 199 & GPL v2+ & 6 \\
\hline PIVOTX & pivotx & Flexible template system to adjust, extend & http://pivotx.net/ & 2006 & 89 & GPL v2+ & 11 \\
\hline Piwigo & piwigo & Photo gallery system for the web & http://piwigo.org/ & 2003 & 236 & GPL v2+ & 19 \\
\hline
\end{tabular}


Table 14: Summary of project sample ( $n=60$; part 3 of 3 )

\begin{tabular}{|c|c|c|c|c|c|c|c|}
\hline Project & $\begin{array}{l}\text { CPE product } \\
\text { (CVE record) }\end{array}$ & Description & Website & $\begin{array}{l}\text { Year } \\
\text { started }\end{array}$ & $\begin{array}{l}\text { Code base } \\
\text { (kLOC) }\end{array}$ & Licenses & \# of CVEs \\
\hline Plone & plone & Enterprise CMS & https://plone.org/ & 2002 & 1248 & GPL v2 & 55 \\
\hline PmWiki & pmwiki & System collaborative creation, maintenance & http://www.pmwiki.org/ & 2004 & & GPL & 7 \\
\hline PrestaShop & prestashop & Professional e-commerce shopping cart & https://www.prestashop.com/ & 2011 & 364 & OSL v3 & 10 \\
\hline PunBB & punbb & Lightweight PHP-powered & http://punbb.informer.com/ & 2008 & 44 & GPL v2+ & 41 \\
\hline Serendipity & serendipity & PHP-powered weblog engine & https://www.s9y.org/ & 2003 & 231 & BSD & 40 \\
\hline SilverStripe & silverstripe & Application framework & https://www.silverstripe.org/ & 2007 & 728 & BSD & 34 \\
\hline SPIP & spip & Web site publishing, collaborative editing & http://www.spip.net/ & 2002 & 144 & GPL v3+ & 24 \\
\hline Textpattern & textpattern & Built-in-tags, code to control presentation & http://textpattern.com/ & 2004 & 117 & GPL & 9 \\
\hline TomatoCart & tomatocart & PHP/Mysql shopping cart & http://www.tomatocart.com/ & 2012 & 407 & GPL v3 & 5 \\
\hline TWiki & twiki & Enterprise wiki and web application platform & http://twiki.org/ & 2002 & 2070 & GPL & 25 \\
\hline TYPO3 & typo3 & Scaleable web application framework & https://typo3.org/ & 2003 & 3810 & GPL v2+ & 96 \\
\hline UseBB & usebb & PHP based bulletin board package & http://www.usebb.net/ & 2004 & 56 & GPL & 9 \\
\hline Vanilla & vanilla forums & Discussion forum & https://vanillaforums.com/en/ & 2005 & 314 & GPL v2+ & 8 \\
\hline webEdition & webedition & Web application framework & http://www.webedition.org/ & 2008 & 197 & No declared & 2 \\
\hline WebGUI & webgui & Web application framework & https://www.webgui.org/ & 2001 & 789 & GPL v2+ & 14 \\
\hline WOLFCMS & wolfcms & Tools necessary for file management & https://www.wolfcms.org/ & 2009 & 38 & GPL v3 & 2 \\
\hline WordPress & wordpress & Software to create a beautiful website. & https://wordpress.org/ & 2003 & 423 & GPL v2+ & 255 \\
\hline XOOPS CMS & xoops & Extensible, object-oriented, dynamic CMS. & http://www.xoops.org/ & 2005 & 8494 & GPL v2+ & 38 \\
\hline ZenPHOTO & zenphoto & The simpler media website CMS & http://www.zenphoto.org/ & 2011 & 170 & GPL v2+ & 19 \\
\hline Zikula & zikula & PHP application framework & http://zikula.org/ & 2010 & 304 & GPL v3+ & 1 \\
\hline
\end{tabular}




\subsection{Manual inspection of small sample}

The method for inspecting the small sample, and the guiding questions that motivated this step, were previously explained in section 6.6. Table 15 is a summary of the ten vulnerabilities in the small sample.

Table 15: Summary of small sample of vulnerabilities

\begin{tabular}{llccccccc}
\hline \multicolumn{1}{c}{ CVE ID } & Project & $\begin{array}{c}\text { Count of Count } \\
\text { vulner- } \\
\text { able } \\
\text { versions }\end{array}$ & $\begin{array}{c}\text { First } \\
\text { refer- } \\
\text { ences }\end{array}$ & $\begin{array}{c}\text { Release } \\
\text { version }\end{array}$ & $\begin{array}{c}\text { First } \\
\text { date } \\
\text { unaffected } \\
\text { version }\end{array}$ & $\begin{array}{c}\text { Release } \\
\text { date }\end{array}$ & $\begin{array}{c}\text { Exposure } \\
\text { time } \\
\text { (days) }\end{array}$ \\
\hline 1 CVE-2014-0122 Moodle & 61 & 3 & 2.0 & $2010-11-24$ & 2.4 .9 & $2014-03-10$ & 1202 \\
\hline 2 CVE-2013-4523 Moodle & 121 & 3 & 1.11 & $2003-09-11$ & 2.3 .10 & $2013-11-18$ & 3721 \\
\hline 3 CVE-2014-0166 WordPress & 98 & 4 & 0.71 & $2003-06-09$ & 3.7 .2 & $2014-04-08$ & 3956 \\
\hline 4 CVE-2012-0827 Drupal & 27 & 1 & 7.0 & $2011-01-05$ & 6.23 & $2012-02-01$ & 392 \\
\hline 5 CVE-2009-2170 Mahara & 27 & 1 & 1.1 .0 & $2008-04-01$ & 1.0 .12 & $2009-06-21$ & 446 \\
\hline 6 CVE-2010-3841 TWiki & 24 & 4 & 4.0 .0 & $2006-02-01$ & 5.0 .1 & $2009-10-10$ & 1347 \\
\hline 7 CVE-2012-0283 DokuWiki & 17 & 7 & $2005-$ & $2005-07-01$ & $2012-$ & $2012-07-13$ & 2569 \\
\hline 8 CVE-2011-1513 e107 & 59 & 4 & 0.7 .0 & $2006-01-16$ & rev12375 & $2011-10-21$ & 2104 \\
\hline 9 CVE-2010-4616 Impress & 35 & 4 & 1 & $2008-04-17$ & 1.2 .4 & $2010-12-22$ & 979 \\
\hline 10 CVE-2012-1590 Drupal & 30 & 7 & 1 & $2011-01-05$ & 7.13 & $2012-05-02$ & 483 \\
\hline
\end{tabular}

All vulnerabilities in the small sample existed for more than a year before being fixed. The exposure time was more than 5 years for four of the vulnerabilities, and more than ten years for two of the vulnerabilities.

Insights from inspecting this sample of CVE records include the following:

(1) Neither the "Publish Date" nor the "Update Date" from CVE Details are useful as proxies for the events in the vulnerability life cycle model. The "Publish Date" appears to match the "Date Entry Created" field of the CVE record in the MITRE Corporation CVE List, and the "Original release date field of the CVE record in the National 
Vulnerability Database. According to the CVE FAQ (MITRE, 2016a):

The "Date Entry Created" date in a CVE Identifier (CVE ID) indicates when the CVE ID was issued to a CVE Numbering Authority (CNA) or published on the CVE List. This date does not indicate when the vulnerability was discovered, shared with the affected vendor, publicly disclosed, or updated in CVE. That information may or may not be included in the description or references of a CVE ID, or in the enhanced information for the CVE ID that is provided in the U.S. National Vulnerability Database (NVD).

Likewise, "Update Date" appears to match the "Last revised" field of the CVE record in the National Vulnerability Database, which is updated when new information is posted to the CVE record. For some CVEs in the small sample, this date was close to the fix event, but for other CVEs, it was months or years later. This is unfortunate, because these two dates are easily harvested from CVE Details summary table reports.

(2a) The release date of the first affected version is an effective proxy for the introduction event. Using online sources, it was possible to identify the release dates for the versions of the software reported as affected by CVE Details, and to select the oldest date as the introduction event.

(2b) The release date of the first unaffected version after introduction of the vulnerability is a useful proxy for the fix event. Using online sources, it was possible to identify the first version of the software released after the introduction but not on the list of affected versions, and to identify the release date of that version.

(3) The number of linked records in the "References for CVE" field varied between 1 and 7. All of the CVE records examined included a security advisory, typically issued on the same day that the fix was available; the format varied widely, from formal records to blog posts. Other references variously included posts to the [oss-security] mailing list 
requesting assignment of a CVE, posts to project mailing lists discussing the vulnerability, posts to other public discussion about the vulnerability, bug reports, commit logs for the commitment of a patch to the project source code that addresses the vulnerability, and blog posts. The formats and content of project records varied widely between different projects.

(4) For some CVEs, the references were informative about the events in the vulnerability lifecycle model. For example, the first public announcement about the vulnerability was typically the same date that a fix was available and included instructions about how to patch or otherwise mitigate the vulnerability. For some CVEs with particularly voluminous and rich references, there was information about how and when the vulnerability was reported, and how the developers responded. For other CVEs with few references, the additional information was sparse.

Unfortunately, there was no reliable proxy for the discovery event that could be consistently harvested from the CVE Details records. There were reliable proxies for the introduction event and the fix event using the list of affected versions and using other online sources to identify release dates of the project's software. With proxies for the introduction event and the fix event, it is possible to test hypotheses about the total exposure time, but with no proxy for the discovery event, it is not possible to test the hypotheses about time to discover or time to fix. The automated data collection techniques of Munzert et al. (2015) can be applied to identify the CVEs affecting a project and the affected versions, but a manual operation will be required to identify the version release dates and the corresponding proxies for the introduction and fix events. 


\subsection{Statistical analysis of full sample}

The method for statistical analysis of the full sample of vulnerabilities was previously explained in section 6.9. Following the advice of Fox (2016, p. 28): “Careful data analysis should begin with inspection of the data" - for this thesis, inspection of the data includes summary statistics, plots, and close examination of project metrics, followed by univariate examination of the explanatory and response variables, then bivariate examination of combinations of variables. Next, we present in detail linear regression analysis of the response variable on the unmodified explanatory variables, and report the results of the hypothesis tests. Following the advice of Fox (2016) and employing the techniques of Fox \& Weisberg (2011), we then present regression diagnostics of the model 1 results, including inspection of residuals. Finally, we explore a series of alternative models, including smoothing the explanatory variables with timeaveraging, and various logarithmic and power transforms of the explanatory and response variables, to investigate the robustness of the hypothesis tests to various modelling assumptions. Finally, we examine effect size and practical significance of the associations.

\subsubsection{Project metrics}

Table 16 is a summary report of the open source project metrics used to compute the explanatory variables (Table 10 in section 6.7) for each of the 1286 vulnerabilities in the full sample, in the month that the vulnerability was fixed. The number of contributors in the month of the fix ranges between 1 and 77, with a median of 10, a sample mean of 19 , and an interquartile range of 29 . The number of code commits to the project source 
code in the month of the fix ranges between 3 and 4686, with a median of 420, a sample mean of 828 , and an interquartile range of 871 . Project source code ranges in size from four kLOC to more than four thousand kLOC, with a median of $309 \mathrm{kLOC}$, a sample mean of $730 \mathrm{kLOC}$, and an interquartile range of $840 \mathrm{kLOC}$. All of these project metrics are right-skewed in distribution, with a small number of relatively large values resulting in a sample mean that is approximately twice the median. The metrics for lines of code added and lines of code removed are even more right-skewed by large outliers, resulting in sample means beyond the third quartile of the distributions. This insight is important because regression results can be sensitive to outliers (Fox, 2016).

Table 16: Project metrics: mean, quartiles, and range

\begin{tabular}{|c|c|c|c|c|c|c|c|c|}
\hline Metrics & $\begin{array}{l}\text { Minimum } \\
\text { value }\end{array}$ & $\begin{array}{c}\text { First } \\
\text { quartile }\end{array}$ & $\begin{array}{l}\text { Second } \\
\text { quartile } \\
\text { (median) }\end{array}$ & $\begin{array}{l}\text { Sample } \\
\text { mean }\end{array}$ & $\begin{array}{l}\text { Third } \\
\text { quartile }\end{array}$ & $\begin{array}{l}\text { Maximum } \\
\text { value }\end{array}$ & $\begin{array}{l}\text { Inter- } \\
\text { quartile } \\
\text { range }\end{array}$ & Range \\
\hline $\begin{array}{l}\text { Contributors/ } \\
\text { month }\end{array}$ & 1 & 4 & 10 & 19 & 33 & 77 & 29 & 76 \\
\hline $\begin{array}{l}\text { Commits/ } \\
\text { month }\end{array}$ & 3 & 183 & 420 & 828 & 1054 & 4686 & 871 & 4683 \\
\hline Comment ratio & 0.07 & 0.16 & 0.24 & 0.23 & 0.30 & 0.42 & 0.23 & 0.35 \\
\hline $\begin{array}{l}\text { Code added/ } \\
\text { month }\end{array}$ & 0 & 3820 & 14300 & 80240 & 40740 & 1608000 & 36920 & 1608000 \\
\hline $\begin{array}{l}\text { Code removed/ } \\
\text { month }\end{array}$ & 0 & 2397 & 9933 & 64490 & 28630 & 1120000 & 26233 & 1120000 \\
\hline $\begin{array}{l}\text { Size of code base } \\
\text { (thousand lines of } \\
\text { code, kLOC) }\end{array}$ & 4.39 & 142.10 & 309.10 & 730.10 & 982.40 & 4441.00 & 840.3 & 4441 \\
\hline
\end{tabular}

Table 17 sorts the sixty projects of the project sample in descending order by size of their source code base. Projects vary widely in size: the source code base of the largest project, XOOPS, is more than five hundred times the size of the source code base of the smallest project, Anchor CMS. 
Table 17: Projects sorted by size of code base (kLOC)

\begin{tabular}{|c|c|c|c|}
\hline Project name & KLOC & Project name & KLOC \\
\hline xoops & 8494 & concrete5 & 279 \\
\hline magento & 8259 & pimcore & 257 \\
\hline typo3 & 3810 & bigtreecms & 251 \\
\hline moodle & 3098 & piwigo & 236 \\
\hline opencms & 2796 & serendipity & 231 \\
\hline ezpublish & 2785 & cmsmadesimplecm & 230 \\
\hline twiki & 2070 & ovidentia & 222 \\
\hline plone & 1248 & oscommerce & 211 \\
\hline impresscms & 1030 & actionapps & 202 \\
\hline ocportal & 806 & pivot & 199 \\
\hline webgui & 789 & webedition & 197 \\
\hline movabletype & 769 & zenphoto & 170 \\
\hline phpbb & 737 & lifetype & 157 \\
\hline silverstripe & 720 & spip & 144 \\
\hline drupal & 704 & impresspages & 138 \\
\hline b2evolution & 686 & textpattern & 117 \\
\hline jaws & 675 & monstra & 110 \\
\hline geeklog & 649 & dokuwiki & 103 \\
\hline e107 & 525 & pivotx & 88.9 \\
\hline mahara & 507 & omeka & 73 \\
\hline mediawiki & 493 & bedita & 61 \\
\hline bitweaver & 441 & usebb & 56 \\
\hline wordpress & 423 & croogo & 52 \\
\hline tomatocart & 407 & habari & 48.2 \\
\hline dotclear & 394 & punbb & 44 \\
\hline prestashop & 364 & wolfcms & 38 \\
\hline joomla & 356 & kajona & 26 \\
\hline vanilla forums & 314 & nibbleblog & 25 \\
\hline gallery & 311 & anchorcms & 16 \\
\hline zikula & 304 & pmwiki & \\
\hline
\end{tabular}

To examine how project metrics vary in time within a project, we selected four projects for closer inspection. We deliberately chose projects of different code base sizes: Plone (Figure 6; a very large project with more than one million lines of code), Drupal (Figure 7; a large project with between 500k and on million lines of code), MediaWiki (Figure 8; a medium project with between 250k and 500k lines of code), and Serendipity (Figure 9; a small project with less than 250k lines of code). 
Plone contributors per month

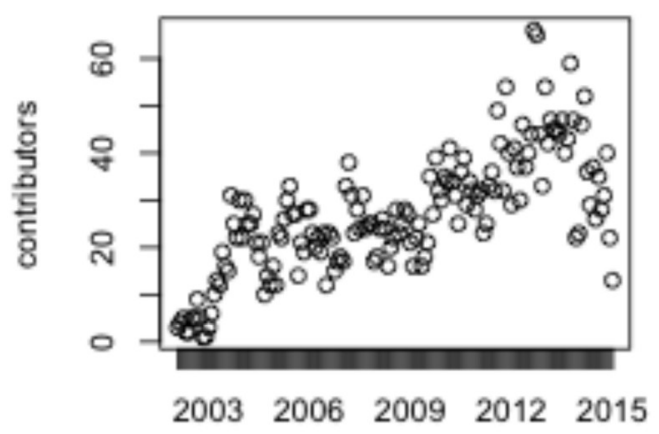

Plone monthly data at open hub

Plone comment ratio

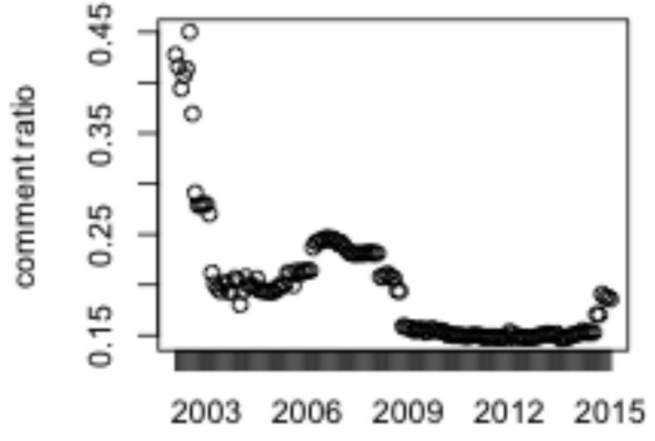

Plone monthly data at open hub

Plone code removed per month

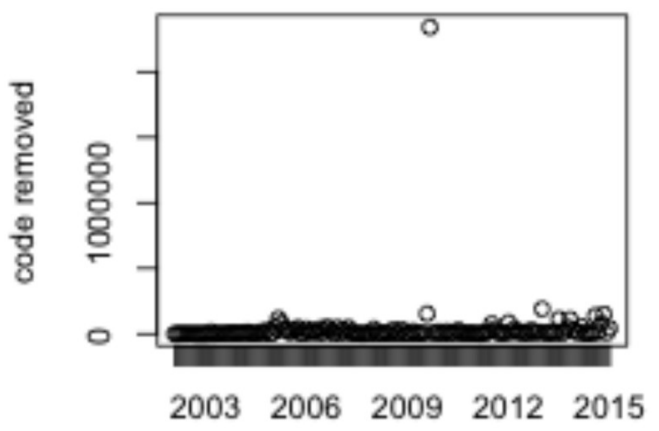

Plone monthly data at open hub
Plone commits per month

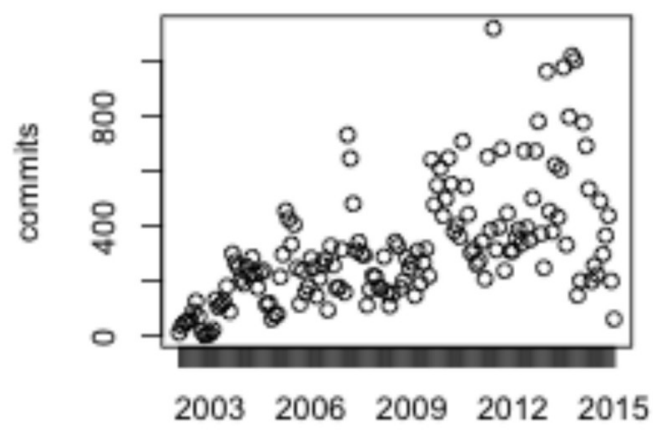

Plone monthly data at open hub

Plone code added per month

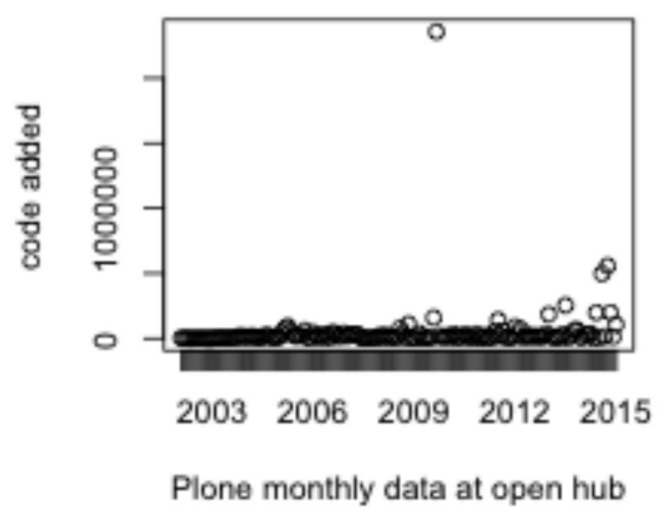

Plone lines of code per month

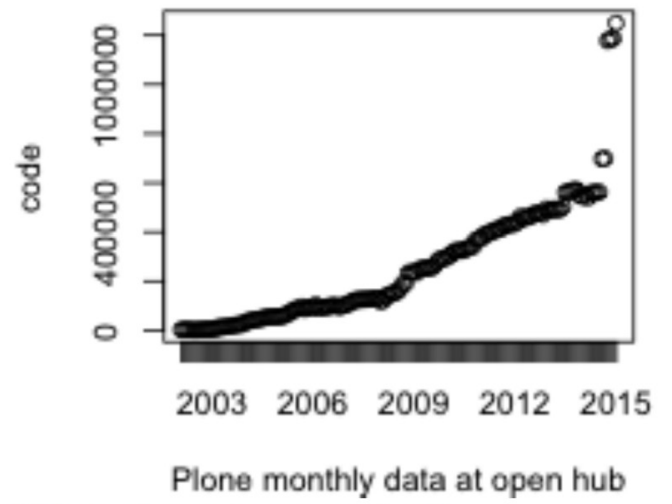

Plone monthly data at open hub

Figure 6: Project metrics of Plone (very large project) 
Drupal contributors per month

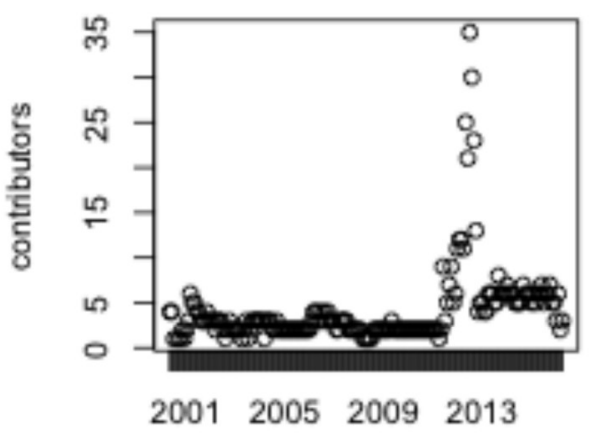

Drupal monthly data at open hub

Drupal comment ratio

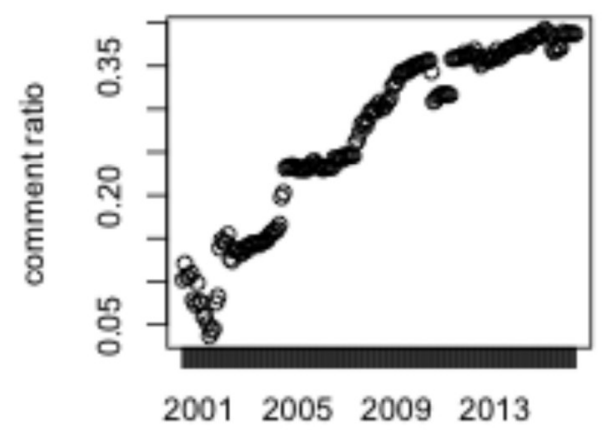

Drupal monthly data at open hub

Drupal code removed per montr

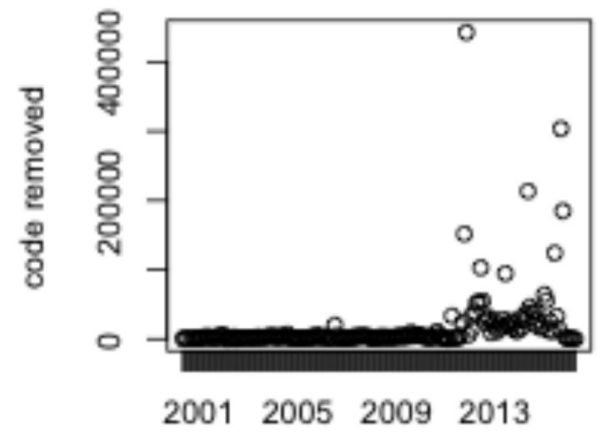

Drupal monthly data at open hub
Drupal commits per month

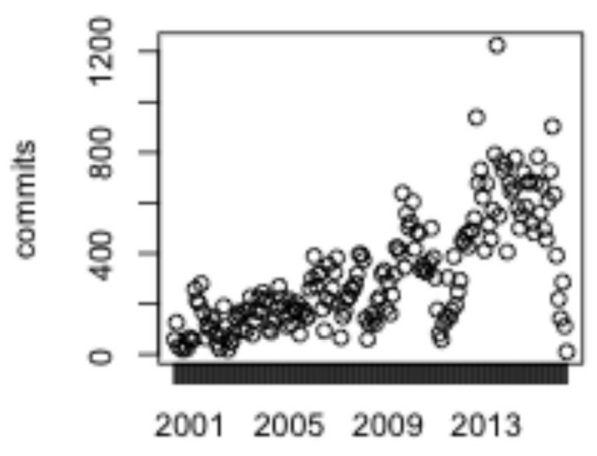

Drupal monthly data at open hub

Drupal code added per month

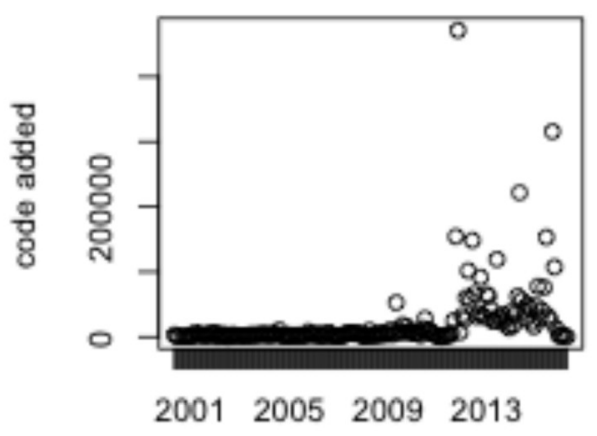

Drupal monthly data at open hub

Drupal lines of code per month

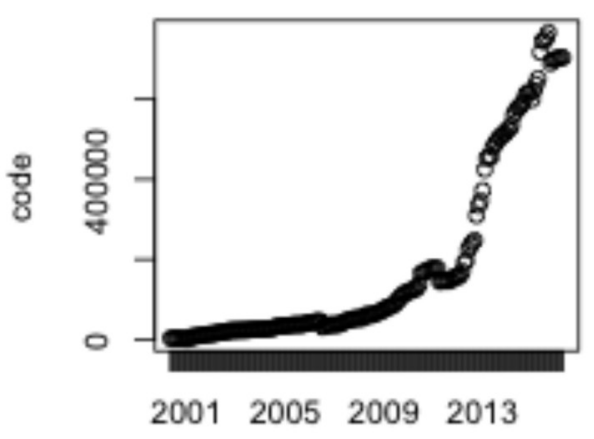

Drupal monthly data at open hub

Figure 7: Project metrics of Drupal (large project) 
Mediawiki contributors per month

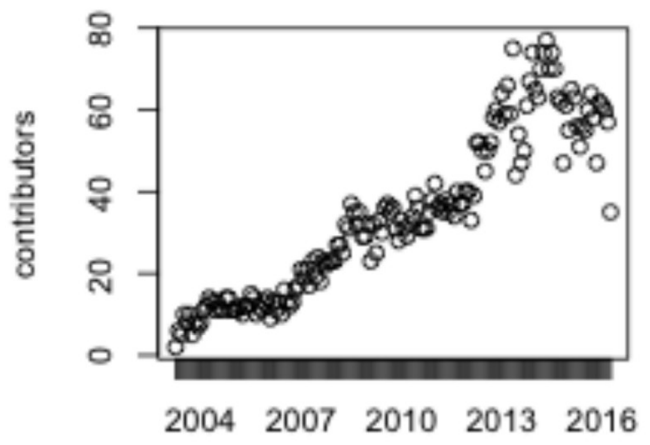

Mediawiki monthly data at open hub

Mediawiki comment_ratio per mont

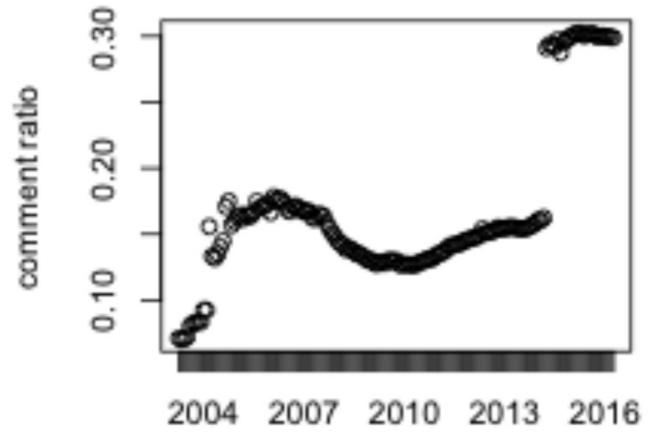

Mediawiki monthly data at open hub

Mediawiki code removed per montl

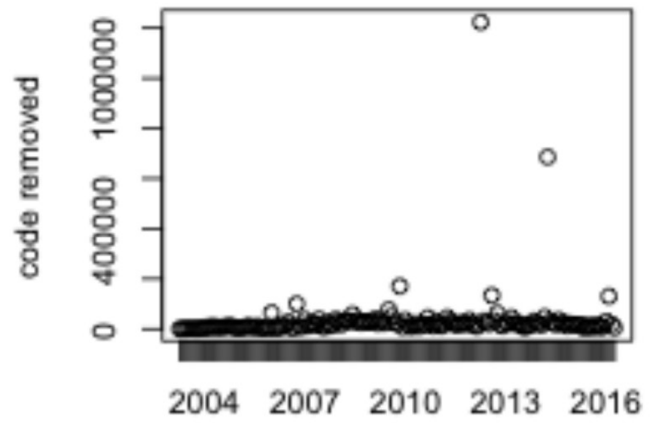

Mediawiki monthly data at open hub
Mediawiki commits per month

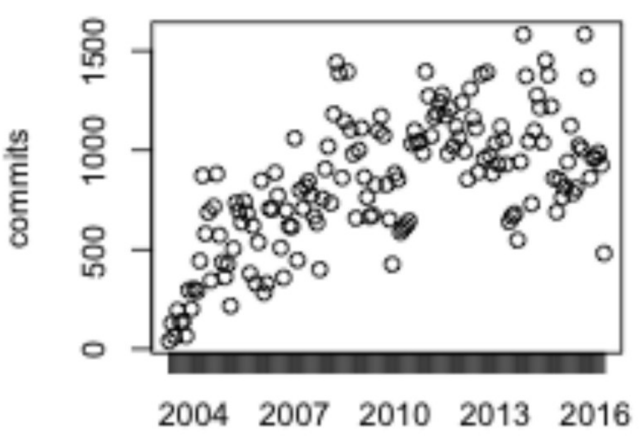

Mediawiki monthly data at open hub

Mediawiki code added per month

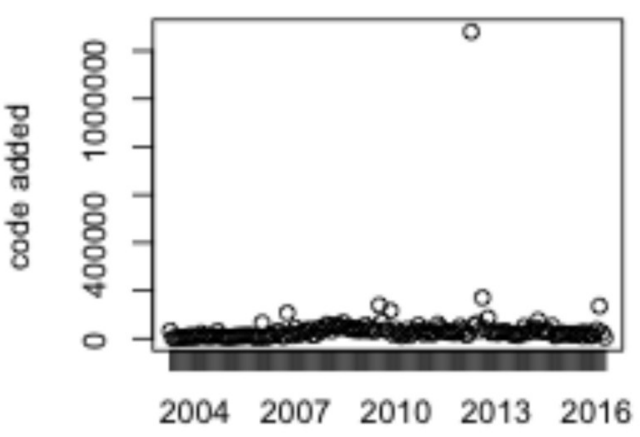

Mediawiki monthly data at open hub

\section{Mediawiki lines of code per month}

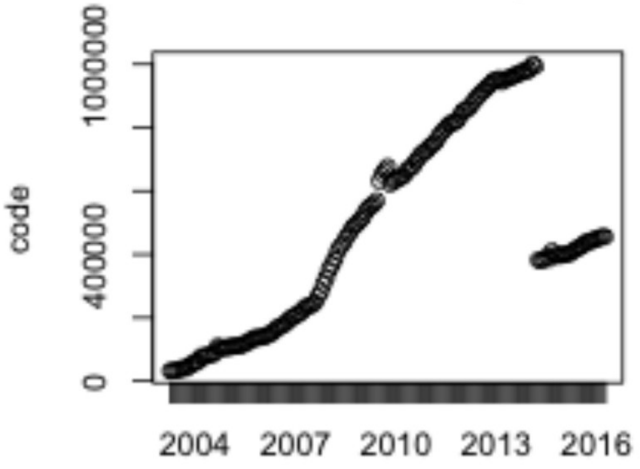

Mediawiki monthly data at open hub

Figure 8: Project metrics of MediaWiki (medium project) 
Serendipity contributors per montr

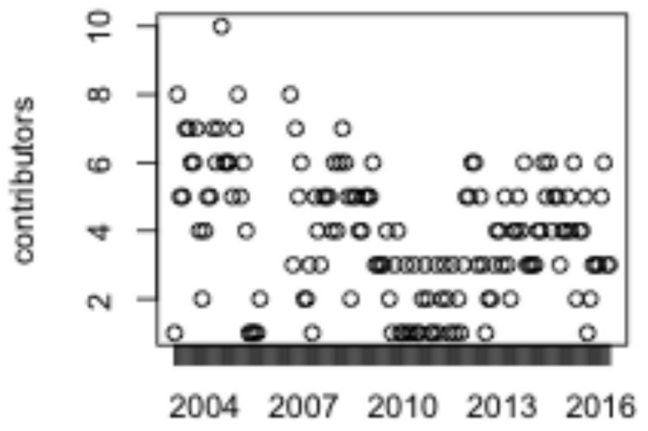

Serendipity monthly data at open hub

Serendipity comment_ratio

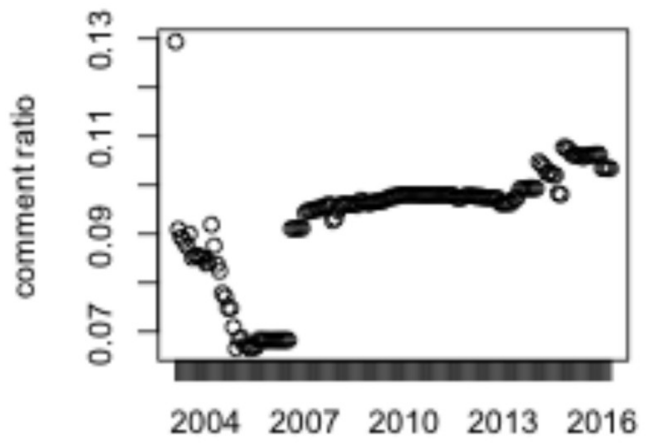

Serendipity monthly data at open hub

\section{Serendipity code removed per mont}

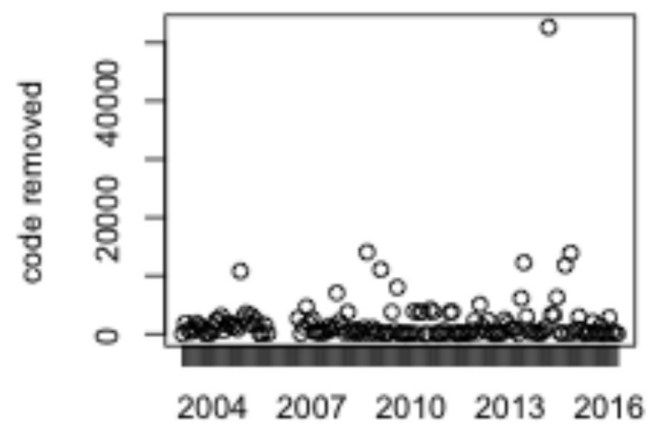

Serendipity monthly data at open hub
Serendipity commits per month

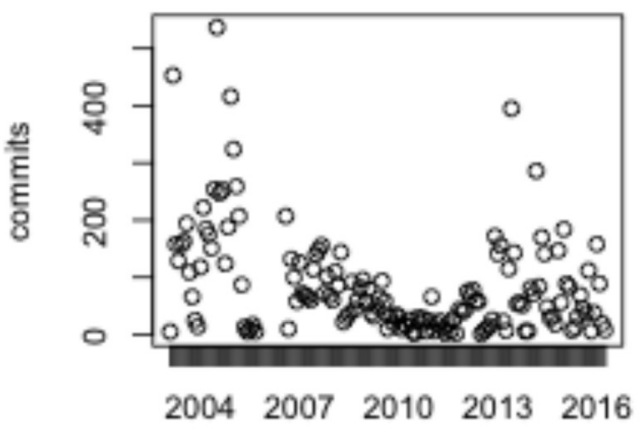

Serendipity monthly data at open hub

Serendipity code added per month

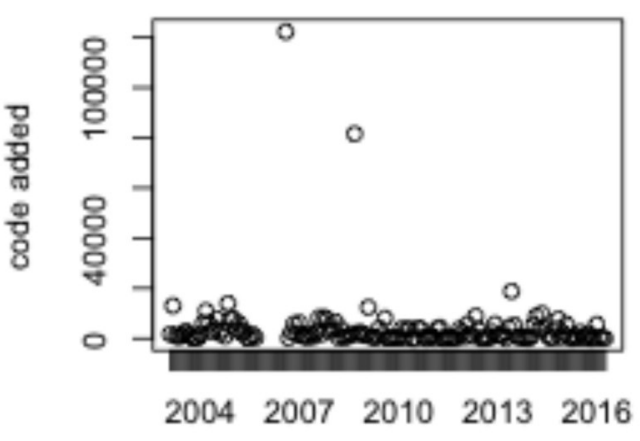

Serendipity monthly data at open hub
Serendipity lines of code per montl

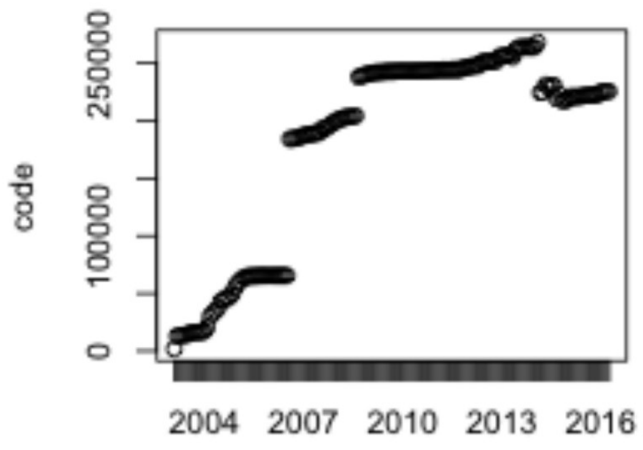

Serendipity monthly data at open hub

Figure 9: Project metrics of Serendipity (small project) 
The plots of project metrics over time are insightful in at least four ways. First, all four projects grow over time in the number of contributors, the number of commits, and the size of the co debase. Second, there is significant month-to-month variation with occasional high spikes, especially in the code added and code removed metrics, but also in the number of contributors and the number of commits. For example, Plone presents a dramatic one-month spike in 2012, with code added and removed both exceeding twenty times the typical monthly value - perhaps an error, or perhaps a one-time re-factoring. Third, there can be abrupt discontinuities, such as removal of half the MediaWiki code base is 2013 - perhaps an outcome of a clean-up operation - or the sudden changes in size of the Serendipity code base, both up and down, occurring in months with unusually large additions or removal of code, evident on other plots. Fourth, month-to-month variations are particularly evident in the smallest project, Serendipity, where the number of contributors in a month may varies between approximately one and eight over the history of the project. Overall, inspection thus far suggests that time is important - for example, the Drupal project in 2014 is larger, more active, and more commented that the Drupal project in 2009. Thus we need to measure the explanatory variables at a point in time relevant to the event of interest. Also, some averaging over time may reduce the noise of month-to-month variations in the project metrics, and may help address outliers.

\subsubsection{Univariate displays}

Figure 10 plots histograms and boxplots for the four explanatory variables (contributor density, commit density, comment ratio, and code churn ratio) and the response variable (time of exposure). 


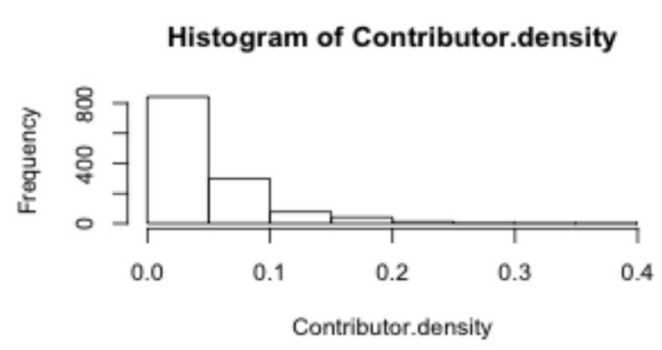

Histogram of Commit.density

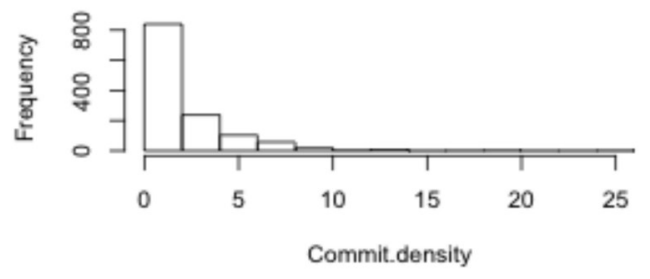

Histogram of Comment.ratio

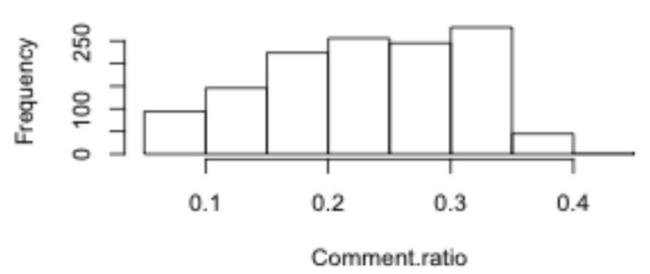

Histogram of Code.churn.ratio

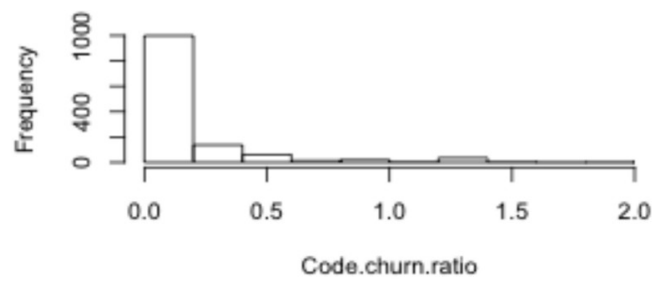

Histogram of Exposure.time

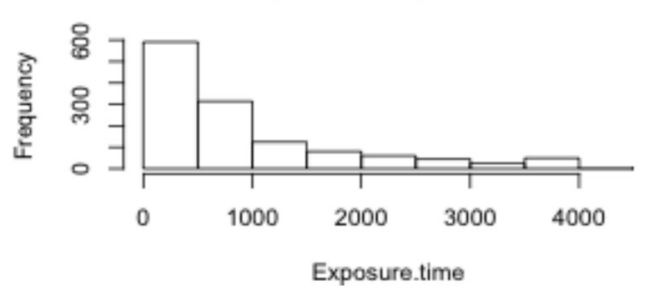

boxplot of Contributor.density

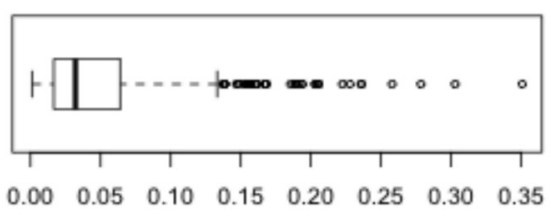

boxplot of Commit.density

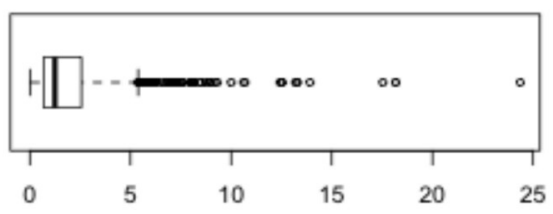

boxplot of Comment.ratio

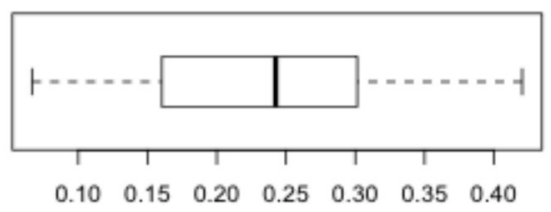

boxplot of Code.churn.ratio

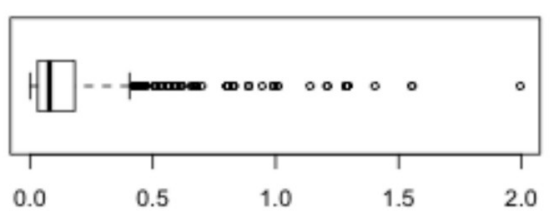

boxplot of Exposure.time

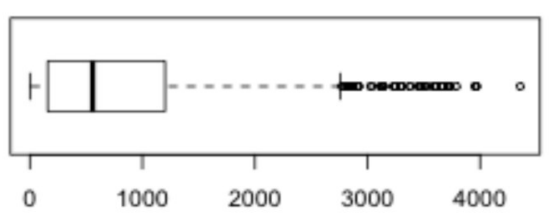

Figure 10: Histograms and boxplots

Copyright (C) 2017 S. M. Monzur Murshed 
Recall that the four explanatory variables are ratios of project metrics (Table 10, addressing the previous observation that projects vary widely in the size of their source code base), in the month of the vulnerability fix event (addressing the previous observation in subsection 7.3.2 that project metrics change significantly over time).

Most variables (except for comment ratio) are right-skewed in distribution, with a relatively large number of smaller values and a long right tail. According to Fox (2016, p. 59), there are at least three reasons to consider transforming skewed distributions to make them more symmetric: (1) highly skewed distributions are difficult to examine and interpret because most observations are confined to a small part of the range of the data, (2) outlying values in the direction of the skew are brought in toward the main body of the data, and unusual values in the opposite direction of the skew can be revealed, and (3) least-squares regression summarizes distributions using means but means are not good summaries of the centre of skewed distributions. Subsection 7.3.8 examines transforms to address skewness.

\subsubsection{Bivariate displays}

Figure 11 is a scatterplot matrix of the bivariate scatterplots for all pairs of variables. Fox (2016) recommends manual inspection of the scatterplot matrix as an informative graphical analog to inspecting the correlation matrix. 
$\begin{array}{lll}0.00 & 0.15 & 0.30\end{array}$
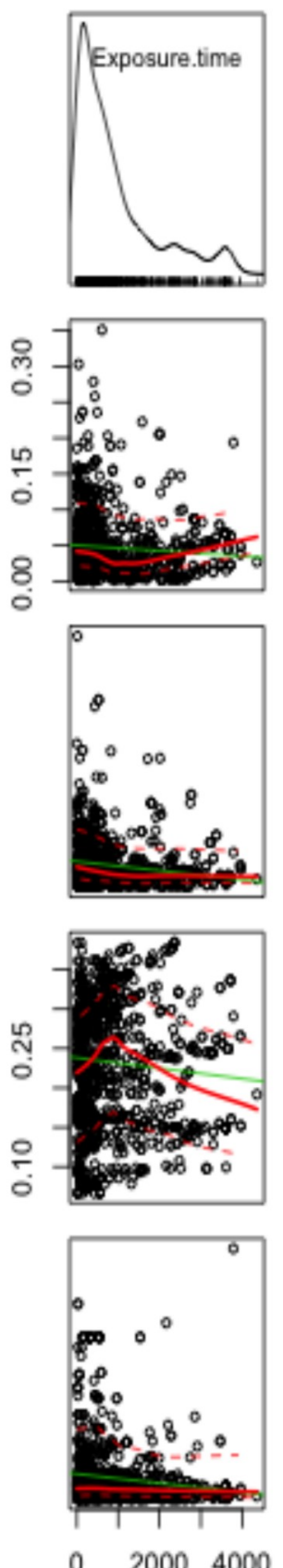

$\begin{array}{lll}0 & 2000 \quad 4000\end{array}$
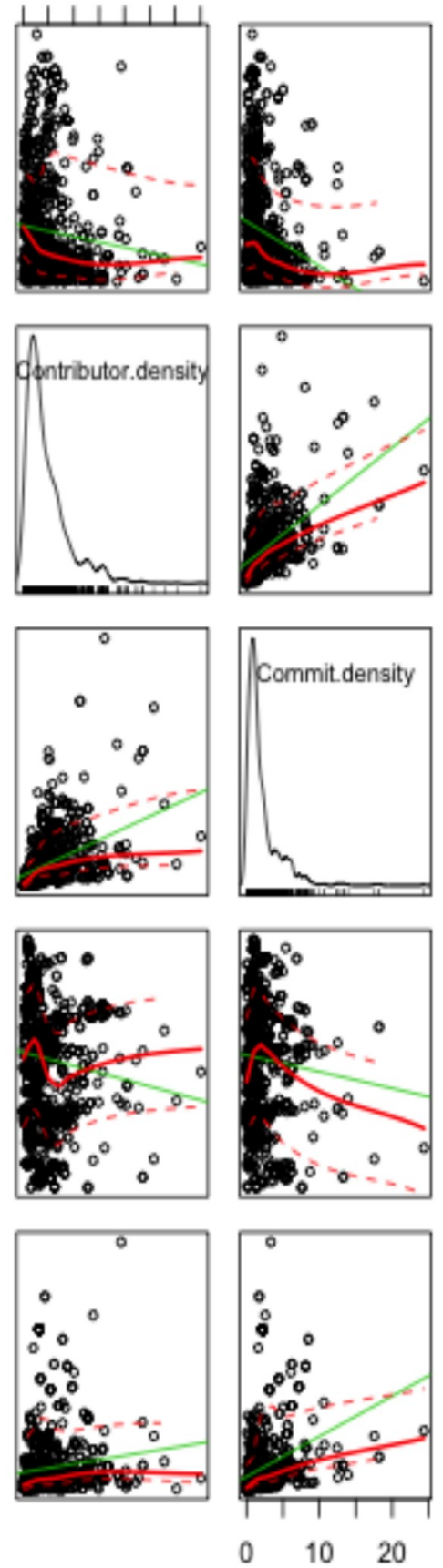

\section{$0.10 \quad 0.25$}
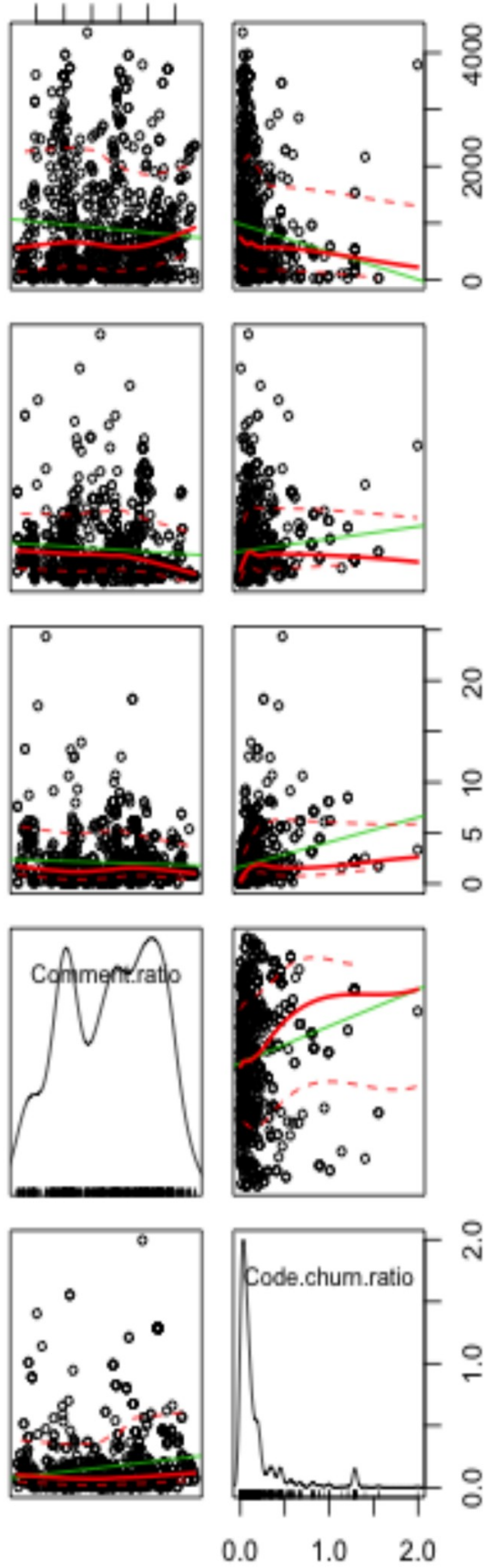

Figure 11: Scatterplot matrix (all variables)

Copyright (C) 2017 S. M. Monzur Murshed 
Fox \& Weisberg (2011) explain the two colours of smoothing lines on the scatter

plots. First, the green straight line is fit by ordinary least squares (OLS) regression.

Second, the solid red curved line is fit using the lowess method (locally weighted scatterplot smoother; Fox, 2016, p. 23) - a form of local linear regression fit to data in the neighbourhood of the focal point, with weights declining as the $\mathrm{x}$ values get farther from the $\mathrm{x}$ value of the focal point. The two red dotted lines show the fraction of the data included in the local regression fit.

The main diagonal of the scatter plot matrix displays a nonparametric density estimation of each variable, with a naive kernel and a rug-plot at the bottom showing the location of data values (Fox, 2016, p. 35). This is similar to the traditional histograms in Figure 10, except with averaging and smoothing. Right-skew is evident for exposure time, contributor density, commit density, and code churn ratio.

The top row of the scatterplot matrix displays the bivariate relationships of exposure time with each explanatory variable. The over-all trends appear to be approximately consistent with the hypotheses at low and medium values of the explanatory variables. This thesis explores linear relationships in subsection 7.3.5, then explores transforms to improve linearity and correct skewness in subsection 7.3.8.

At high values of the explanatory variables, the over-all trend is less clear. The local smoothing line for code churn ratio diverges sharply up at high values - possibly from influential outliers that may be addressed by a transform to correct skewness and improve linearity (explored in subsection 7.3.8), or it could suggest a possible curvilinear or polynomial relationship, such as is typical with some economic models of decreasing 
returns. In a curvilinear model, either low levels or high levels of code churn could be associated high high exposure times - the former because of low activity (vulnerabilities not found and fixed) and the latter because of high activity (too much change for finding and fixing vulnerabilities). Further exploration of curvilinear relationships with quadratic or polynomial models is an opportunity for future research.

\subsubsection{Summary statistics and correlation}

Table 18 presents descriptive statistics and correlation coefficients.

Table 18: Summary statistics and correlation coefficients

\begin{tabular}{llccccccccc}
\hline Variable & Min. & $\begin{array}{c}\text { First } \\
\text { quartile }\end{array}$ & $\begin{array}{c}\text { Second } \\
\text { quartile } \\
\text { (median) }\end{array}$ & Mean & $\begin{array}{c}\text { Third } \\
\text { quartile }\end{array}$ & Max. & $\mathbf{1}$ & $\mathbf{2}$ & $\mathbf{3}$ & $\mathbf{4}$ \\
\hline 1 Contributor.density & 0.000 & 0.017 & 0.032 & 0.047 & 0.064 & 0.350 & & & \\
2 & Commit.density & 0.006 & 0.686 & 1.244 & 2.076 & 2.567 & 24.360 & 0.44 & & \\
3 & Comment.ratio & 0.067 & 0.160 & 0.243 & 0.232 & 0.301 & 0.420 & -0.09 & -0.06 & \\
4 & Code.churn.ratio & 0.000 & 31.23 & 79.37 & 169.1 & 182.5 & 1995 & 0.11 & 0.28 & 0.16 \\
5 Exposure.time & 1.000 & 158.5 & 558.0 & 893.0 & 1202 & 4357 & -0.09 & -0.19 & -0.08 & -0.13 \\
\hline
\end{tabular}

Fox (2016, p. 59) warns that the mean of a skewed distribution is not a good summary of the centre. Four of the five variables are right-skewed, thus the median is preferred here as the measure of central tendency. Likewise, the Internationale range (the difference between the third quartile and the first quartile) is preferred here as the measure of variation of the untransformed variables.

The values of the correlation matrix are commonly interpreted as evidence of whether or not simple linear associations exist between pairs of variables, and the direction of association (Fox, 2016). All four explanatory variables are negatively correlated with exposure time (consistent with the hypotheses). Contributor density 
appears to be correlated with commit density $(\mathrm{r}=0.44)$ - a possible indication of multicollinearity that could negatively impact the stability of regression results. Likewise, comment ratio appears to be correlated with code churn ratio $(\mathrm{r}=0.28)$. We address the possibility of multicollinearity in subsection 7.3.6, and explore the effect of removing variables in subsequent analysis.

\subsubsection{Regression results (models 1 and 2)}

Table 19 presents the results of linearly regressing the response variable of exposure time $\left(t_{e}\right)$ on the four explanatory variables (model 1), contributor density $\left(x_{1}\right)$, commit density $\left(x_{2}\right)$, comment ratio $\left(x_{3}\right)$, and code churn ratio $\left(x_{4}\right)$.

Table 19: Regression results (no time-averaging)

\begin{tabular}{lll}
\hline Variable & $\begin{array}{l}\text { Model 1 } \\
\text { (all variables) } \\
(\mathbf{n}=1268)\end{array}$ & $\begin{array}{l}\text { Model 2 } \\
(\text { most significant variables only) } \\
(\mathbf{n}-\mathbf{1 2 7 0})\end{array}$ \\
\hline Intercept & $1324.3^{* * *}$ & $1322.1^{* * *}$ \\
Contributor.density & -314.76 & \\
Commit.density & $-69.184^{* * *}$ & $-80.820^{* * *}$ \\
Comment.ratio & $-961.10^{* *}$ & $-1106.5^{* * *}$ \\
Code.churn.ratio & $-0.2687^{*}$ & \\
$\mathrm{R}^{2}$ & 0.05 & 0.04 \\
\hline${ }^{*} \mathrm{p}<0.05 ;{ }^{* *}<0.01 ;{ }^{* * *} \mathrm{p}<0.001$ & & \\
\hline
\end{tabular}

We test each hypothesis in the standard way for linear regression (Fox, 2016; q.v., Eisenhardt \& Tabrizi, 1995) by inspecting the p-values and sign of the regression coefficients. For each hypothesis, H1c through $\mathrm{H} 4 \mathrm{c}$, we reject the null hypothesis if and only if two conditions are met: (1) the p-value of the regression coefficient is below a critical threshold $(\mathrm{p}<0.05$, indicated by one asterisk in the table of regression results, 
although $\mathrm{p}<0.01$ with at least two asterisks is preferred) and (2) the regression coefficient is of the predicted sign (negative). We interpret rejection of the null hypothesis as support for the alternative hypothesis - that is, support for the hypothesis that we are testing for.

In model 1, hypothesis H1c that higher contributor density is associated with shorter exposure time is not supported; the p-value is above the critical threshold thus the regression coefficient is not statistically significant. Hypothesis $\mathrm{H} 2 \mathrm{c}$, that more commit density is associated with lower vulnerability exposure time, is strongly supported; the pvalue of the commit density coefficient is well below the critical threshold $(p<0.001)$ and the coefficient has predicted sign (negative). Hypothesis $\mathrm{H} 3 \mathrm{c}$ that higher comment ratio is associated with lower exposure time is also supported with a low $p$-value $(\mathrm{p}<0.01)$. Hypothesis $\mathrm{H} 4 \mathrm{c}$, that higher code churn ratio is associated with lower exposure time, is weakly supported with a p-value slightly below the standard critical threshold $(\mathrm{p}<0.5)$.

Model 2, also shown in Table 19, regresses the response variable of exposure time $\left(t_{e}\right)$ on two explanatory variables - commit density $\left(x_{2}\right)$ and comment ratio $\left(x_{3}\right)$. There are two motivations for running model 2 with fewer explanatory variables. First, following standard procedures for hypothesis testing with regression analysis (Fox, 2016), we re-run the regression after removing the explanatory variables that were not statistically significant. Second, removing contributor density addresses possible multicollinearity between contributor density and commit density, and removing code churn ratio addresses possible multicollinearity between comment ratio and code churn ratio. Both $\mathrm{H} 2 \mathrm{c}$ and $\mathrm{H} 3 \mathrm{c}$ are supported in model $2(\mathrm{p}<001)$. 


\subsubsection{Regression diagnostics (model 1)}

According to Fox (2016, p. 266), regression diagnostics “are often the difference between a crude, mechanical data analysis and a careful nuanced analysis that accurately describes the data and therefore supports meaningful interpretation of them." Previous subsections have identified several ways in which the simple linear regression of model 1 and 2 may be inadequate, including month-to-month variability in project metrics (subsection 7.3.1), right-skewness of the response variable and some explanatory variables (subsections 7.3.2 and 7.3.3), and mixed evidence about linearity of associations (subsection 7.3.3). Prior to addressing these deficiencies with time averaging of project metrics in subsection 7.3.7 and a series of transformations in subsection 7.3.8, this subsection examines the residuals of model 1 for further insights.

Figure 12 displays a set of residual plots for model 1. The bottom diagnostic graph is a plot of residuals versus the fitted values - "the most common diagnostic graph in linear regression" (Fox \& Weisberg, 2011, p. 288). The top four diagnostic graphs

plot residuals versus each of the predictors. Fox \& Weisberg (2011, p. 288) write:

If a linear model is correctly specified, then the Pearson residuals are independent of the fitted values and the predictors, and these graphs should be null plots, with no systematic features - in the sense that the conditional distribution of the residuals (on the vertical axis of the graph) should not change with the fitted values or with a predictor (on the horizontal axis). The presence of systematic features generally implies failure of one or more assumptions of the model. Of interest in these plots are nonlinear trends, trends in variation across the graph, and isolated points. 


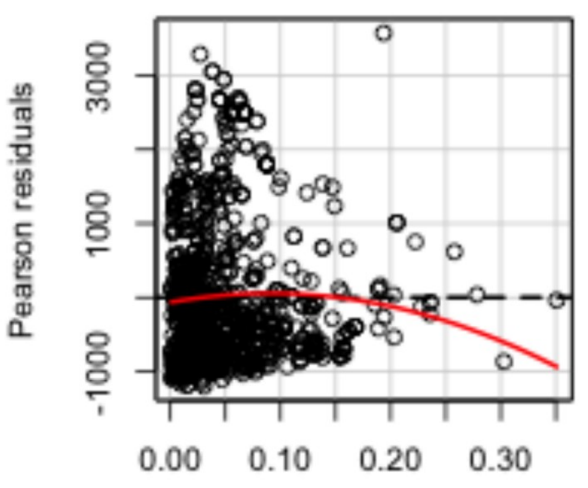

Contributor.density
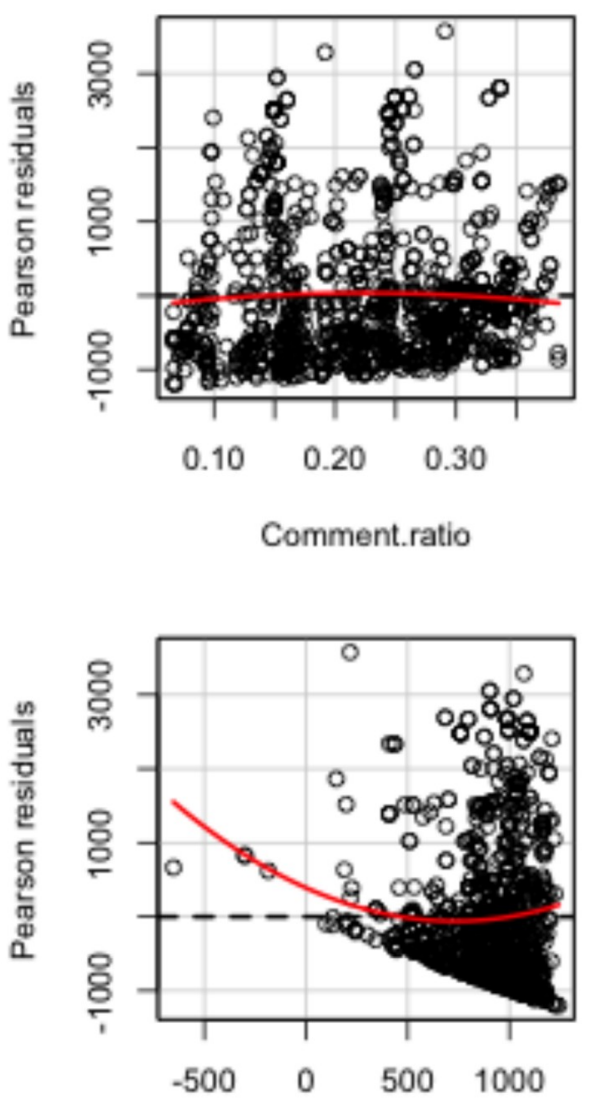

Fitted values
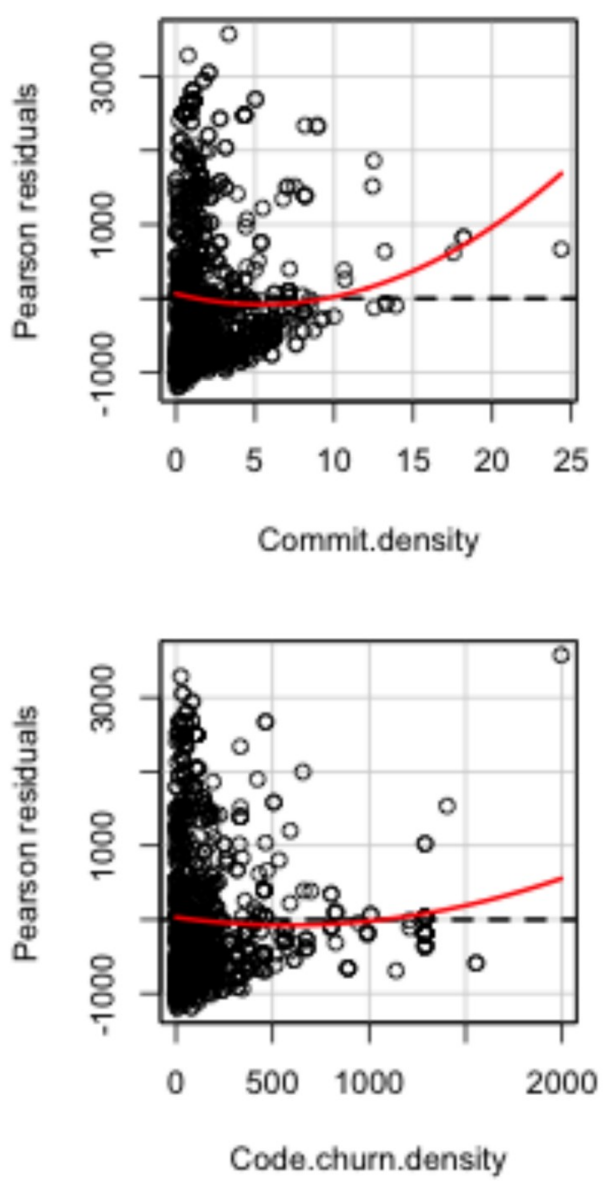

Figure 12: Residual plots for model 1 
There are several insights from inspecting Figure 12. First, with the exception of comment ratio, the $\mathrm{x}$ axis is unbalanced for all variables. This will be addressed in subsection 7.3.8 with transformations to correct for right-skew. Second, the plot of residuals versus fitted values has a curved trend (concave up), suggesting that the model is not adequate to describe the data; this was expected from prior observations about bivariate associations. Third, there appears to be non-constant error, growing larger with larger fitted values, and with smaller values of contributor density, commit density, and code churn ratio, which are expected to be associated with larger fitted values; nonconstant error is also addressable with transformations (Fox, 2016) in subsection 7.3.8. Fourth, there are isolated points, including fitted values with negative time, that may result from month-to-month variation in project metrics, and may be addressed by time averaging. Subsequent subsections will address these inadequacies.

\subsubsection{Time-averaging}

Next, we examine the effect of smoothing the explanatory variables with timeaveraging over three months, six months, and twelve months. Previously in subsection 7.3.1, we had observed large month-to-month variation in some project metrics, which could result in noise and spurious influential outliers in the explanatory variables.

In our base model examined in the previous subsection, the explanatory variables were assigned in the month of the fix event. With a time-averaging window of width $w$, the explanatory variable is the average over $w$ consecutive months, with the last month of the sequence being the month of the fix event. Larger windows will be more effective at reducing noise, but will be less sensitive to real short-term changes. Also, the number of 
usable observations decreases with larger windows because our analysis ignores missing values; if any datum is missing within the time window, that observation is not used. Larger windows provide more possible opportunities for missing values.

Table 20 presents the regression results with all explanatory variables with timeaveraging of the explanatory variables over 3 months (model 3), 6 months (model 4), and 12 months (model 5), and restates the results of model 1 from Table 19 with no timeaveraging. The results are similar to those of model 1, except that $\mathrm{H} 4 \mathrm{c}$ is not supported in models 3, 4, and 5. All four models support $\mathrm{H} 2 \mathrm{c}(\mathrm{p}<0.001)$ and $\mathrm{H} 3 \mathrm{c}(\mathrm{p}<0.01$, and $\mathrm{p}<0.001$ for model 5).

Table 20: Regression results (all explanatory variables; time-averaging)

\begin{tabular}{|c|c|c|c|c|}
\hline Variable & $\begin{array}{l}\text { Model } 1 \\
\text { (1-month) } \\
(n=1268)\end{array}$ & $\begin{array}{l}\text { Model } 3 \\
\text { (3-month) } \\
(n=1245)\end{array}$ & $\begin{array}{l}\text { Model } 4 \\
\text { (6-month) } \\
(n=1228)\end{array}$ & $\begin{array}{l}\text { Model } 5 \\
\text { (12-month) } \\
(n=1188)\end{array}$ \\
\hline Intercept & $1324.4^{* * *}$ & $1264.0^{* * *}$ & $1311.5^{* * *}$ & $1394.9 * * *$ \\
\hline Contributor.density & -314.8 & 336.0 & 769.9 & 881.5 \\
\hline Commit.density & $-69.18^{* * *}$ & $-85.40 * * *$ & $-87.86^{* * *}$ & $-93.46^{* * *}$ \\
\hline Comment.ratio & $-961.1^{* *}$ & $-1039.3 * *$ & -1040.4 ** & $-1272.5^{* * *}$ \\
\hline Code.churn.ratio & $-0.2687^{*}$ & 0.1489 & -0.1906 & -0.2558 \\
\hline $\mathrm{R}^{2}$ & 0.05 & 0.03 & 0.03 & 0.04 \\
\hline
\end{tabular}

Table 21 presents the results of regressing the response variable of exposure time on commit density and comment ratio - the two statistically explanatory variables in Table 20 - with time averaging over 3 months (model 6), 6 months (model 7), and 12 months (model 8), and the restated the results of model 2 with no time averaging, previously reported in Table 19. All four models support $\mathrm{H} 2 \mathrm{c}(\mathrm{p}<0.001)$ and $\mathrm{H} 3 \mathrm{c}$ ( $\mathrm{p}<0.01$ in model 6 , and $\mathrm{p}<0.001$ in models 2,7 , and 8 ). 
Table 21: Regression results (significant variables only; time averaging)

\begin{tabular}{lllll}
\hline Variable & $\begin{array}{l}\text { Model 2 } \\
\text { (Monthly) } \\
(\mathbf{n = 1 2 7 0 )}\end{array}$ & $\begin{array}{l}\text { Model 6 } \\
\text { (Quarterly) } \\
(\mathbf{n}=1247)\end{array}$ & $\begin{array}{l}\text { Model 7 } \\
\mathbf{( 6 ~ M o n t h s )} \\
(\mathbf{n}=1230)\end{array}$ & $\begin{array}{l}\text { Model 8 } \\
\text { (12 Months) } \\
(\mathbf{n}=1190)\end{array}$ \\
\hline Intercept & $1322.1^{* * *}$ & $1284.0^{* * *}$ & $1333.2^{* * *}$ & $1411.5^{* * *}$ \\
Commit.density & $-80.82^{* * *}$ & $-78.51^{* * *}$ & $-84.73^{* * *}$ & $-90.14^{* * *}$ \\
Comment.ratio & $-1106.5^{* * *}$ & $-1002.0^{* *}$ & $-1155.6^{* * *}$ & $-1382.6^{* * *}$ \\
$\mathrm{R}^{2}$ & 0.04 & 0.03 & 0.03 & 0.03 \\
\hline${ }^{*} \mathrm{p}<0.05 ;{ }^{* *}<0.01 ;{ }^{* * *} \mathrm{p}<0.001$ & & & \\
\hline
\end{tabular}

Overall, the results of hypothesis tests are robust to time averaging of the explanatory variables. The 6-month window appears to be effective by providing some smoothing while retaining most observations.

\subsubsection{Transformations}

Next, we examine a series of transformations on the response variable and the explanatory variables, individually and in combination. According to Fox (2016, ch. 4), transformations can often facilitate the examination and statistical modelling of data, in particular, by correcting skewness of variables and improving linearity of associations. This analysis serves two purposes. First it is a way of exploring the robustness of the hypothesis tests to various modelling assumptions; robust results would be relatively insensitive to changes in modelling decisions. Second, it may produce better estimates of associations between variables by addressing the issues identified in subsection 7.3.6.

We begin with the response variable of exposure time. Subsections 7.3.2, 7.3.3, 7.3.4 previously reported that the distribution is right-skewed (see also Figure 10 and Figure 11), and thus difficult to examine and interpret (because most observations are confined to a small part of the range of the data). This can be problematic for linear 
regression, which will be sensitive to outlying values in the right tail, and employs mean values as the centres of distributions. Right skew can be corrected by transformations descending what Tukey (1977) called the ladder of powers (Fox, 2016, ch. 4), first to logarithms, or further to $1 / \mathrm{x}, 1 / \mathrm{x}^{2}$, and to lower powers (i.e., higher roots). A second issue is improving linearity between the explanatory variables and exposure time. Subsections 7.3.3 and 7.3.6 previously reported mixed evidence on linearity - some suggestion of linearity in the bivariate plots of Figure 11, but also concerns with the linear residuals in Figure 12. The bulging rule of Mosteller \& Tukey (1977), as described by Fox (2016, pp. 64-69), suggests that simple monotone nonlinearity of a "bulge down and to the left" - such as observed in the scatterplot of exposure time and contributor density and the scatterplot of exposure time and commit density, can often be improved by transformations descending the ladder of powers for either X or $\mathrm{Y}$, or both. Likewise, a bulge down, possibly suggested by the scatterplots of exposure time and comment ratio and code churn ratio, could be improved by transformations descending the ladder of powers for Y (with no transformation of X). Fortunately, these rules-ofthumb all indicate the same transformation - a logarithm or root of the response variable (exposure time). Fox \& Wiesberg (2011, p. 127) write: “The single most important transformation of a strictly positive variable is the logarithmic transformation" - our starting point. We also examine the response variable transformations identified by two other approaches, described below.

Fox \& Wiesberg (2011, pp. 303-306) describe a set of R implementations of the Box-Cox approach (Box \& Cox, 1964) to selecting a transformation of the response 
variable in regression. The Box-Cox approach identifies the power transformation with the maximum likelihood to produce residuals that are as close to normally distributed as possible. For model 4, for example, the Box-Cox approach recommends a power transform with a lambda of 1.9 , i.e., $\mathrm{X}->\left(\left(\mathrm{X}^{1.9}-1\right) / 1.9\right)$, where $\mathrm{X}$ is exposure time.

Alternatively, Fox \& Wiesberg (2011, pp. 315-317) describe an R implementation of the spread-level plot approach (Tukey, 1977) to suggest a spread-stabilizing power transformation of the response variable based on comparing the absolute Studentized residuals to the log of the fitted values. For model 4, for example, the spread-level plot approach recommends a power transform with a lambda of -0.245 , i.e., $\mathrm{X}->\left(\left(\mathrm{X}^{-0.245}-1\right) /\right.$ $(-0.245))$, where $\mathrm{X}$ is exposure time.

The following tables present the regression results implementing three different transformations of exposure time - a base-10 logarithm (Table 22) suggested by the Fox (2016) rules-of-thumb and the Mosteller \& Tukey (1977) bulging rule, a Box-Cox power transformation (Table 23) using the approach of Box \& Cox (1964), and a spread-level power transformation (Table 24) using the approach of Tukey (1977). Figure 13 presents parallel boxplots comparing the three transformations - an approach recommended by Fox \& Wiesberg (2011) to compare transforms with respect the impact on skewness. 
Table 22: Regression results (log transform of response variable)

\begin{tabular}{lllll}
\hline Variable & $\begin{array}{l}\text { Model 9 } \\
\text { (Monthly) } \\
(\mathbf{n = 1 2 6 8 )}\end{array}$ & $\begin{array}{l}\text { Model 10 } \\
(\text { Quarterly) } \\
(\mathbf{n}=1245)\end{array}$ & $\begin{array}{l}\text { Model 11 } \\
(\mathbf{6} \text { Months) } \\
(\mathbf{n}=1228)\end{array}$ & $\begin{array}{l}\text { Model 12 } \\
(\mathbf{1 2} \text { Months) } \\
(\mathbf{n}=1188)\end{array}$ \\
\hline Intercept & $2.789^{* * *}$ & $2.773^{* * *}$ & 2.805 & $2.848^{* * *}$ \\
Contributor.density & $-1.05186^{*}$ & $-1.25478^{* *}$ & $-1.16831^{*}$ & $-1.00706^{*}$ \\
Commit.density & $-0.05467^{* * *}$ & $-0.05916^{* * *}$ & $-0.06086^{* * *}$ & $-0.07511^{* * *}$ \\
Comment.ratio & 0.11758 & 0.08451 & 0.03292 & -0.06497 \\
Code.churn.ratio & -0.00009031 & 0.00011163 & 0.00001654 & 0.00003607 \\
$\mathrm{R}^{2}$ & 0.07 & 0.05 & 0.05 & 0.05 \\
\hline${ }^{*} \mathrm{p}<0.05 ;{ }^{* *}<0.01 ;{ }^{* * *} \mathrm{p}<0.001$ & & & \\
\hline
\end{tabular}

Table 23: Regression results (Box-Cox power transform of response variable)

\begin{tabular}{lllll}
\hline Variable & $\begin{array}{l}\text { Model 13 } \\
\text { (Monthly) } \\
(\mathbf{n = 1 2 6 8 )}\end{array}$ & $\begin{array}{l}\text { Model 14 } \\
\text { (Quarterly) } \\
(\mathbf{n = 1 2 4 5 )}\end{array}$ & $\begin{array}{l}\text { Model 15 } \\
\text { (6 Months) } \\
(\mathbf{n}=1228)\end{array}$ & $\begin{array}{l}\text { Model 16 } \\
\text { (12 Months) } \\
\text { (n=1188) }\end{array}$ \\
\hline Intercept & $3.5175^{* * *}$ & $3.49210^{* * *}$ & $3.53404^{* * *}$ & $3.59415^{* * *}$ \\
Contributor.density & $-1.28633^{*}$ & $-1.33496^{*}$ & -1.15272. & -0.95091 \\
Commit.density & $-0.06915^{* * *}$ & $-0.07849^{* * *}$ & $-0.08123^{* * *}$ & $-0.09652^{* * *}$ \\
Comment.ratio & -0.01942 & -0.07049 & -0.12091 & -0.26310 \\
Code.churn.ratio & -0.00016814 & 0.0001141 & -0.00004983 & -0.00005417 \\
$\mathrm{R}^{2}$ & 0.06 & 0.05 & 0.04 & 0.05 \\
\hline${ }^{*} \mathrm{p}<0.05 ;{ }^{* *}<0.01 ;{ }^{* * *} \mathrm{p}<0.001$ & & & \\
\hline
\end{tabular}

Table 24: Regression results (spread-level power transform of response variable)

\begin{tabular}{lllll}
\hline Variable & $\begin{array}{l}\text { Model 17 } \\
\text { (Monthly) } \\
(\mathbf{n}=\mathbf{1 2 6 8})\end{array}$ & $\begin{array}{l}\text { Model 18 } \\
\text { (Quarterly) } \\
(\mathbf{n}=1245)\end{array}$ & $\begin{array}{l}\text { Model 19 } \\
(\mathbf{6} \text { Months) } \\
(\mathbf{n}=1228)\end{array}$ & $\begin{array}{l}\text { Model 20 } \\
(\mathbf{1 2} \text { Months) } \\
(\mathbf{n}=1188)\end{array}$ \\
\hline Intercept & $0.60241^{* * *}$ & $0.05438^{* * *}$ & $0.219044^{* * *}$ & $0.15270^{* * *}$ \\
Contributor.density & $0.12653^{*}$ & $0.17114^{* * *}$ & $0.23332^{* *}$ & $0.20748^{* *}$ \\
Commit.density & $0.0066139^{* * *}$ & $0.0054494^{* * *}$ & $0.0090305^{* * *}$ & $0.0109404^{* * *}$ \\
Comment.ratio & -0.019258 & -0.023667 & -0.0214619 & -0.0095947 \\
Code.churn.ratio & 0.000008480 & -0.00002185 & -0.00001499 & -0.00002527 \\
$\mathrm{R}^{2}$ & 0.07 & 0.06 & 0.05 & 0.06 \\
\hline${ }^{*} \mathrm{p}<0.05 ;{ }^{* *}<0.01 ;{ }^{* * *} \mathrm{p}<0.001$ & & & \\
\hline
\end{tabular}



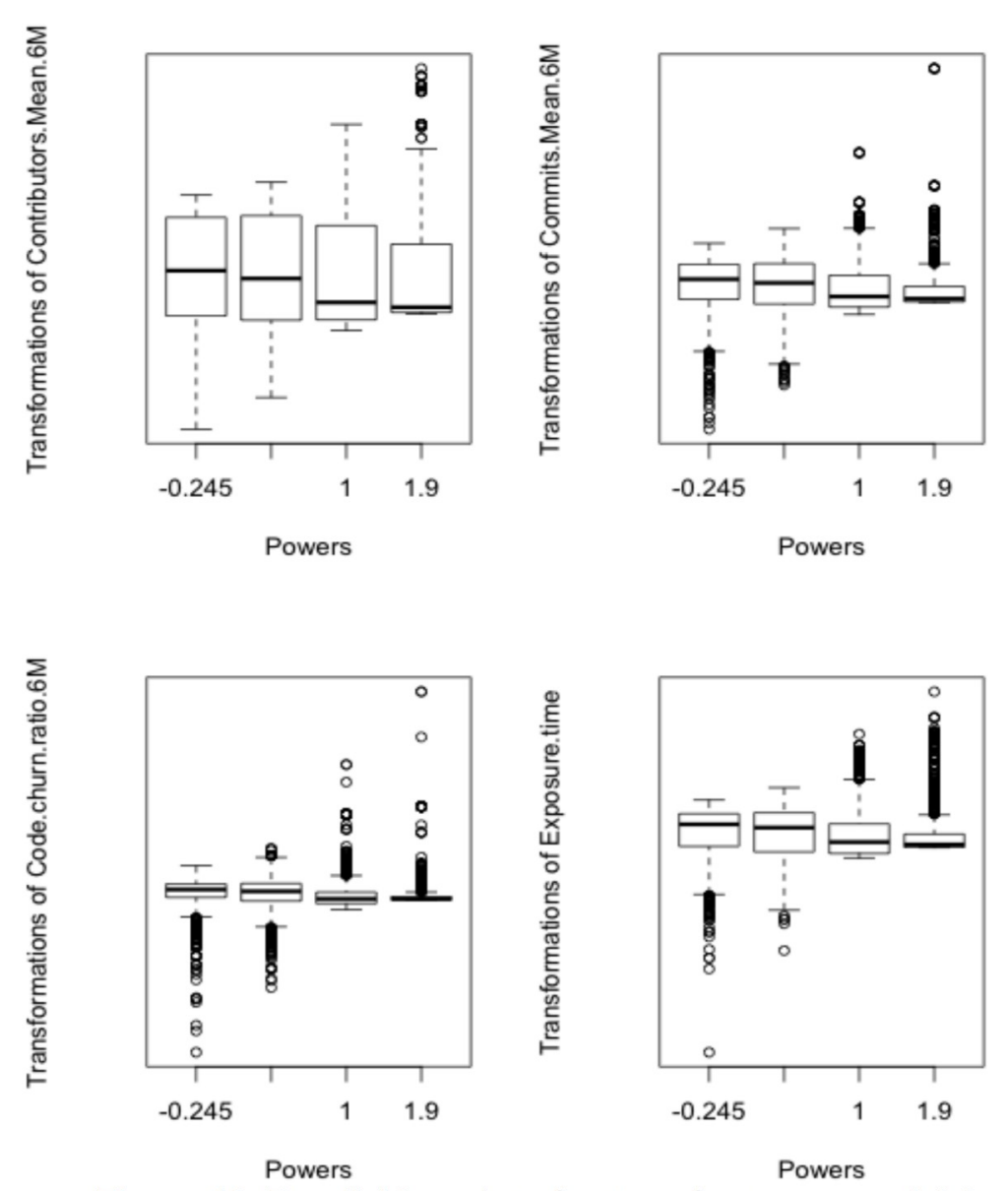

Figure 13: Parallel boxplots for transforms on model 4

In all three sets of transformed models, across all time-averaging windows, the coefficient for commit density is statistically significant $(\mathrm{p}<0.001)$. For the logarithm transformation (Table 22) and the Box-Cox transformation with lambda $=1.9$ (Table 23), the sign is negative as predicted by hypothesis H2c. For the spread-level transformation with lambda $=-0.245$ (Table 24), the sign is positive - the opposite direction predicted by H2c. Figure 13 reveals the cause of this reversal - that the spread-level transformation over-compensates for skewness $\mathrm{n}$ the sample, resulting in left-skew rather than 
symmetry. We interpret this is a strong support for hypothesis $\mathrm{H} 2 \mathrm{c}$, that more commit density is associated with shorter vulnerability exposure time, which appears to be robust to variations in modelling assumptions.

The coefficient for contributor density is statistically significant and with the predicted sign in the logarithmic transformation for all four time windows $(\mathrm{p}<0.05$ or $\mathrm{p}<0.01)$ and two of the time windows for the Box-Cox transformation $(\mathrm{p}<0.05)$. It is statistically significant $(\mathrm{p}<0.05$ or $\mathrm{p}<0.01)$ but of the opposite sign for the spread level transformation (which over-compensates for skewness). We interpret this cautiously as some (weak) support for H1c.

Of the three transformations plotted in Figure 13, the log transformation of the response variable provides the best skewness correction, and is selected for further analysis. Table 25 presents the results of regressing the base 10 logarithm of exposure time on contributor density and commit density - the two statistically significant explanatory variables in Table 22 . H3c is strongly supported $(\mathrm{p}<0.001)$; H1c is also supported $(\mathrm{p}<0.05$ or $\mathrm{p}<0.01)$.

Table 25: Regression results (log transform of response variable; sig. variables only)

\begin{tabular}{lllll}
\hline Variable & $\begin{array}{l}\text { Model 21 } \\
\text { (Monthly) } \\
(\mathbf{n = 1 2 7 0 )}\end{array}$ & $\begin{array}{l}\text { Model 22 } \\
\text { (Quarterly) } \\
(\mathbf{n}=1247)\end{array}$ & $\begin{array}{l}\text { Model 23 } \\
\mathbf{( 6 \text { Months) }} \\
(\mathbf{n}=1230)\end{array}$ & $\begin{array}{l}\text { Model 24 } \\
(\mathbf{1 2} \text { Months) } \\
(\mathbf{n}=1190)\end{array}$ \\
\hline Intercept & $2.8084^{* * *}$ & $2.8072^{* * *}$ & $2.8156^{* * *}$ & $2.8367^{* * *}$ \\
Contributor.density & $-1.0603^{*}$ & $-1.2442^{* *}$ & $-1.1741^{*}$ & $-0.99526^{*}$ \\
Commit.density & $-0.05780^{* * *}$ & $-0.056553^{* * *}$ & $-0.06061^{* * *}$ & $-0.07429^{* * *}$ \\
$\mathrm{R}^{2}$ & 0.07 & 0.05 & 0.05 & 0.05 \\
\hline${ }^{*} \mathrm{p}<0.05^{* *}<0.01^{* * * *} \mathrm{p}<0.001$ & & & \\
\hline
\end{tabular}


Next, we consider the explanatory variables. The Fox (2016) rules of thumb previously suggested transformations to descend the ladder of powers to correct the rightskew variables (that is, contributor density, commit density, and code churn ratio) and to improve the linearity of associations "bulging" to the bottom-left (that is, contributor density and commit density). Table 26 (all variables) and Table 27 (significant variables only) present the regression results with base 10 logarithm transformations on the response variable of exposure time and the explanatory variables of contributor density, commit density, and code churn ratio, and the untransformed comment ration.

Table 26: Regression results (log transform of all variables)

\begin{tabular}{|c|c|c|c|c|}
\hline Variable & $\begin{array}{l}\text { Model } 25 \\
\text { (Monthly) } \\
(n=1268)\end{array}$ & $\begin{array}{l}\text { Model } 26 \\
\text { (Quarterly) } \\
(n=1245)\end{array}$ & $\begin{array}{l}\text { Model } 27 \\
(6 \text { Months) } \\
(n=1228)\end{array}$ & $\begin{array}{l}\text { Model } 28 \\
\text { (12 Months) } \\
(n=1188)\end{array}$ \\
\hline Intercept & $2.4391^{* * *}$ & $2.0400 * * *$ & $2.1850 * * *$ & $2.2934 * * *$ \\
\hline $\log 10$ (Contributor.density) & -0.06113 & $-0.12778 *$ & -0.10918 & -0.05632 \\
\hline log10(Commit.density) & $-0.22143^{* * *}$ & $-0.26908^{* * *}$ & $-0.26345^{* * *}$ & $-0.29416 * * *$ \\
\hline Comment.ratio & 0.22033 & 0.05677 & 0.05533 & 0.05632 \\
\hline log10(Code.churn.ratio) & 0.03895 & $0.21006^{* * *}$ & $0.15199 * * *$ & $0.14010^{* *}$ \\
\hline $\mathrm{R}^{2}$ & 0.04 & 0.05 & 0.04 & 0.04 \\
\hline
\end{tabular}

Table 27: Regression results (log transform of significant variables only)

\begin{tabular}{lllll}
\hline Variable & $\begin{array}{l}\text { Model 29 } \\
\text { (Monthly) } \\
(\mathbf{n = 1 2 7 0 )}\end{array}$ & $\begin{array}{l}\text { Model 30 } \\
\text { (Quarterly) } \\
(\mathbf{n}=1247)\end{array}$ & $\begin{array}{l}\text { Model 31 } \\
\mathbf{( 6 \text { Months) }} \\
(\mathbf{n}=1230)\end{array}$ & $\begin{array}{l}\text { Model 32 } \\
(\mathbf{1 2} \text { Months) } \\
(\mathbf{n}=1190)\end{array}$ \\
\hline Intercept & $2.5710^{* * *}$ & $2.2412^{* * *}$ & $2.3577^{* * *}$ & $2.3903^{* * *}$ \\
$\log 10$ (Commit.density) & $-0.25773^{* * *}$ & $-0.34225^{* * *}$ & $-0.32732^{* * *}$ & $-0.32834^{* * *}$ \\
$\log 10$ (Code.churn.ratio) & $0.04641^{* * *}$ & $0.21506^{* * *}$ & $0.15677^{* * *}$ & $0.14237^{* *}$ \\
$\mathrm{R}^{2}$ & 0.04 & 0.05 & 0.04 & 0.04 \\
\hline${ }^{*} \mathrm{p}<0.05^{* *}<0.01 ;{ }^{* * *} \mathrm{p}<0.001$ & & & \\
\hline
\end{tabular}


As a final step in this section about transformations, we consider the role of unusual data and the robustness of the hypothesis test results to the removal of influential data points. Fox (2016, p. 267) writes: "Unusual data are problematic in linear models fit by least squares because they can unduly influence the results of the analysis and because their presence may be a signal that the model fails to capture important characteristics of the data." The pages that follow specifically re-examine three models: model 11 (base-10 logarithm transformation of the response variable with all explanatory variables included; 6-month time-averaging window), model 23 (base-10 logarithm transformation of the response variable with only statistically-significant explanatory variables; 6-month timeaveraging window), and model 27 (base-10 logarithm transformation of the response variable and three explanatory variables; 6-month time-averaging window).

Fox (2016, p. 276) recommends constructing influence plots (also called "bubble plots") that combine measures of discrepancy, leverage, and influence on one graphical display, with studentized residuals (discrepancy) on the vertical axis, hat-values (leverage) on the horizontal axis, and circles of an area proportional to Cook's Distance (influence). Horizontal reference lines at residuals of 0 and $+/-2$ and vertical reference lines at hat-values of $2 \mathrm{~h}$ and $3 \mathrm{~h}$ suggest extreme values for further inspection or removal.

On the pages that follow, Figure 14, Figure 15, and Figure 16 display the influence plots of model 11, model 23 , and model 27 , respectively, along with variant models that remove the 100 most influential observations, and Table 28, Table 29, and Table 30 compare the regression results. In each variant model with influential observations removed, the results of the hypothesis tests at $\mathrm{p}<0.01$ are unchanged. 


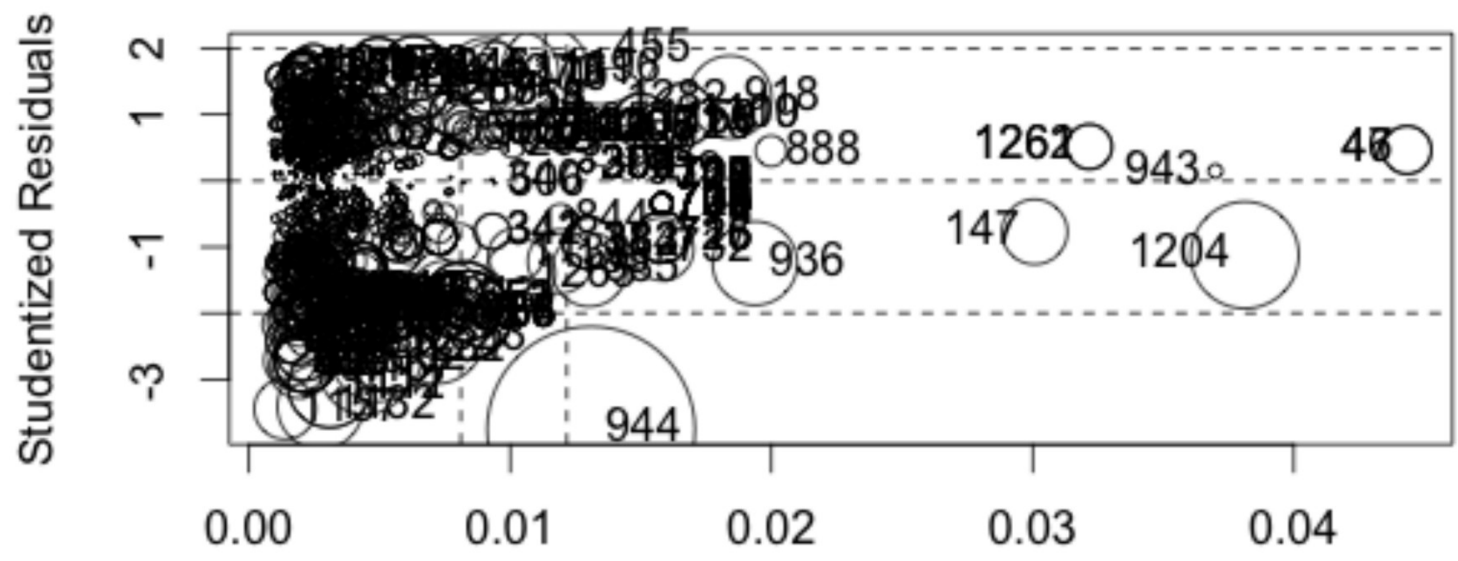

\section{Hat-Values}

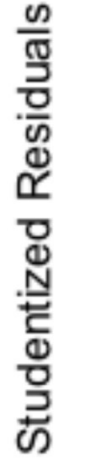

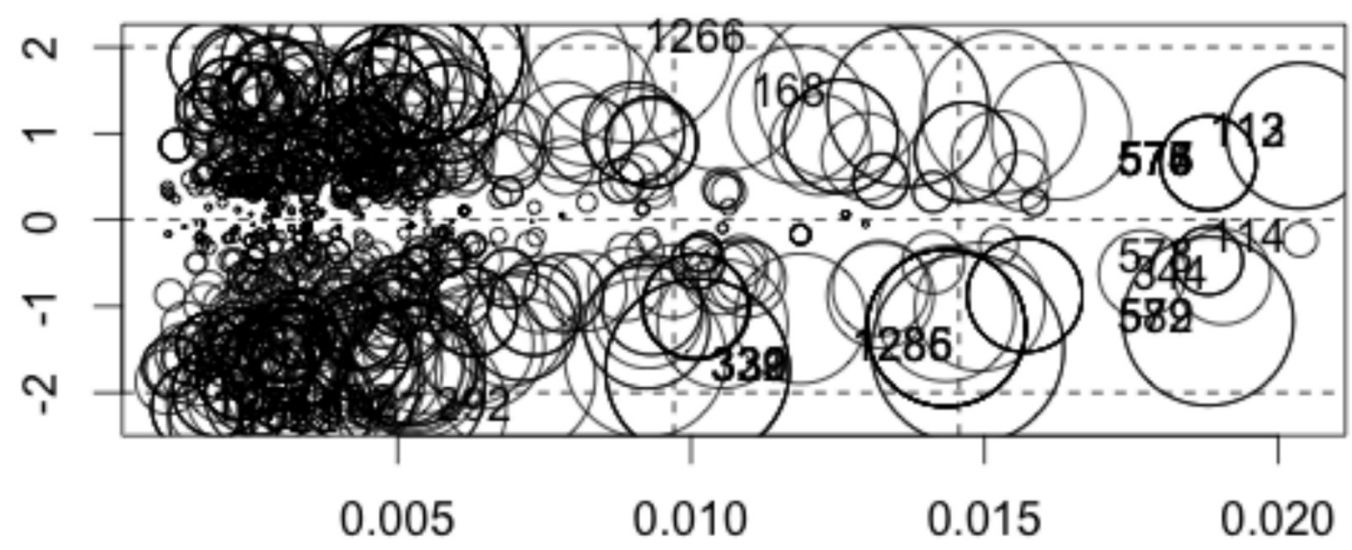

Hat-Values

Figure 14: Influence plots of model 11 and 11* (with 100 observations removed)

Table 28: Regression results of Model 11* (with unusual observations removed)

\begin{tabular}{lll}
\hline Variable & $\begin{array}{l}\text { Model 11 } \\
\text { (6 Months; } \mathbf{n = 1 2 2 8 )}\end{array}$ & $\begin{array}{l}\text { Model 11* } \\
\text { (6 Months; } \mathbf{n = 1 0 8 9 )}\end{array}$ \\
\hline Intercept & $2.805^{* * *}$ & $2.866^{* * *}$ \\
Contributor.density & $-1.16831^{*}$ & -0.09875 \\
Commit.density & $-0.06086^{* * *}$ & $-0.07685^{* * *}$ \\
Comment.ratio & 0.03292 & -0.03956 \\
Code.churn.ratio & 0.00001654 & 0.15586 \\
$\mathrm{R}^{2}$ & 0.05 & 0.05 \\
\hline${ }^{*} \mathrm{p}<0.05 ;{ }^{* *}<0.01 ;{ }^{* * *} \mathrm{p}<0.001$ & & \\
\hline
\end{tabular}

Copyright (C) 2017 S. M. Monzur Murshed 


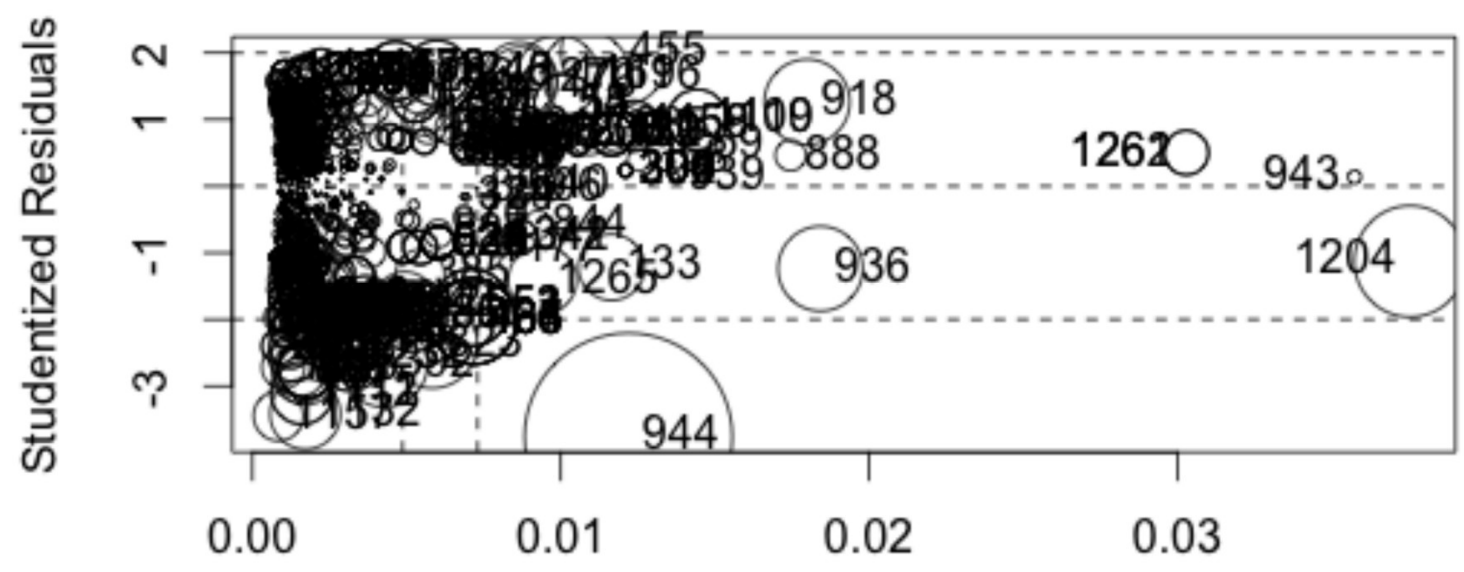

Hat-Values

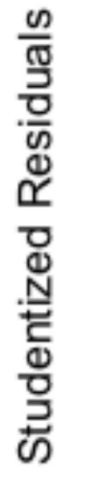

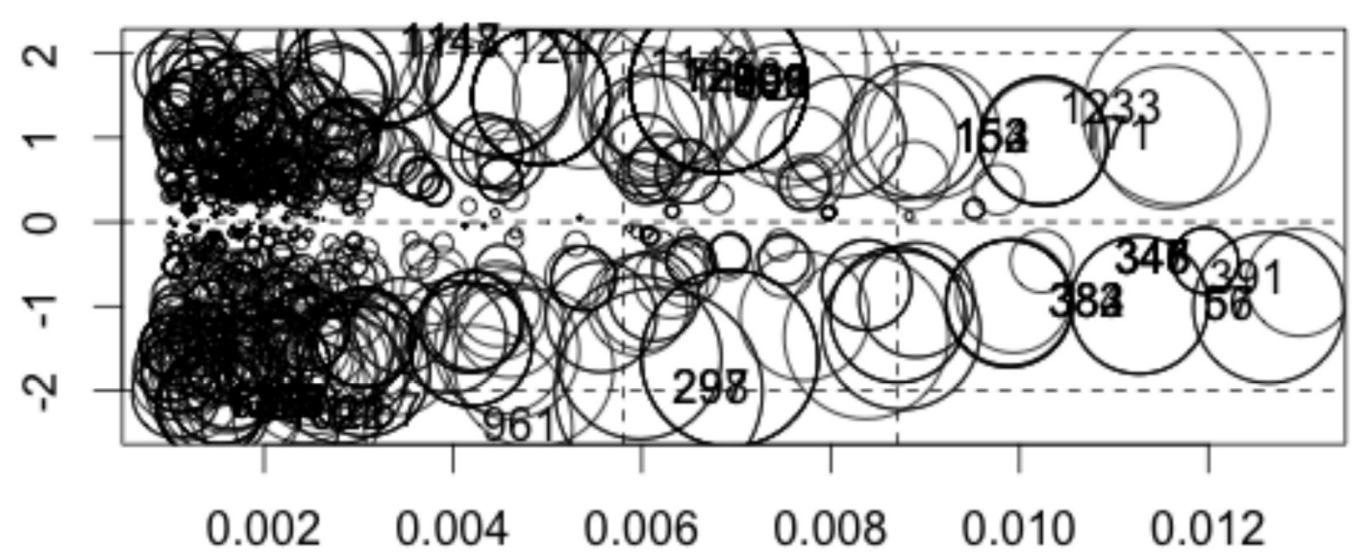

Hat-Values

Figure 15: Influence plots of model 23 and $23^{*}$ (with 100 observations removed)

Table 29: Regression results of Model 23* (with unusual observations removed)

\begin{tabular}{lll}
\hline Variable & $\begin{array}{l}\text { Model 23 } \\
\text { (6 Months; } \mathbf{n = 1 2 3 0 )}\end{array}$ & $\begin{array}{l}\text { Model 23* } \\
\text { (6 Months; } \mathbf{n = 1 0 3 1 ) ~}\end{array}$ \\
\hline Intercept & $2.8156^{* * *}$ & $2.91105^{* * *}$ \\
Contributor.density & $-1.1741^{*}$ & -0.20152 \\
Commit.density & $-0.06061^{* * *}$ & $-0.10045^{* * *}$ \\
$\mathrm{R}^{2}$ & 0.05 & 0.08 \\
\hline${ }^{*} \mathrm{p}<0.05 ;{ }^{* *}<0.01 ;{ }^{* * *} \mathrm{p}<0.001$ & & \\
\hline
\end{tabular}



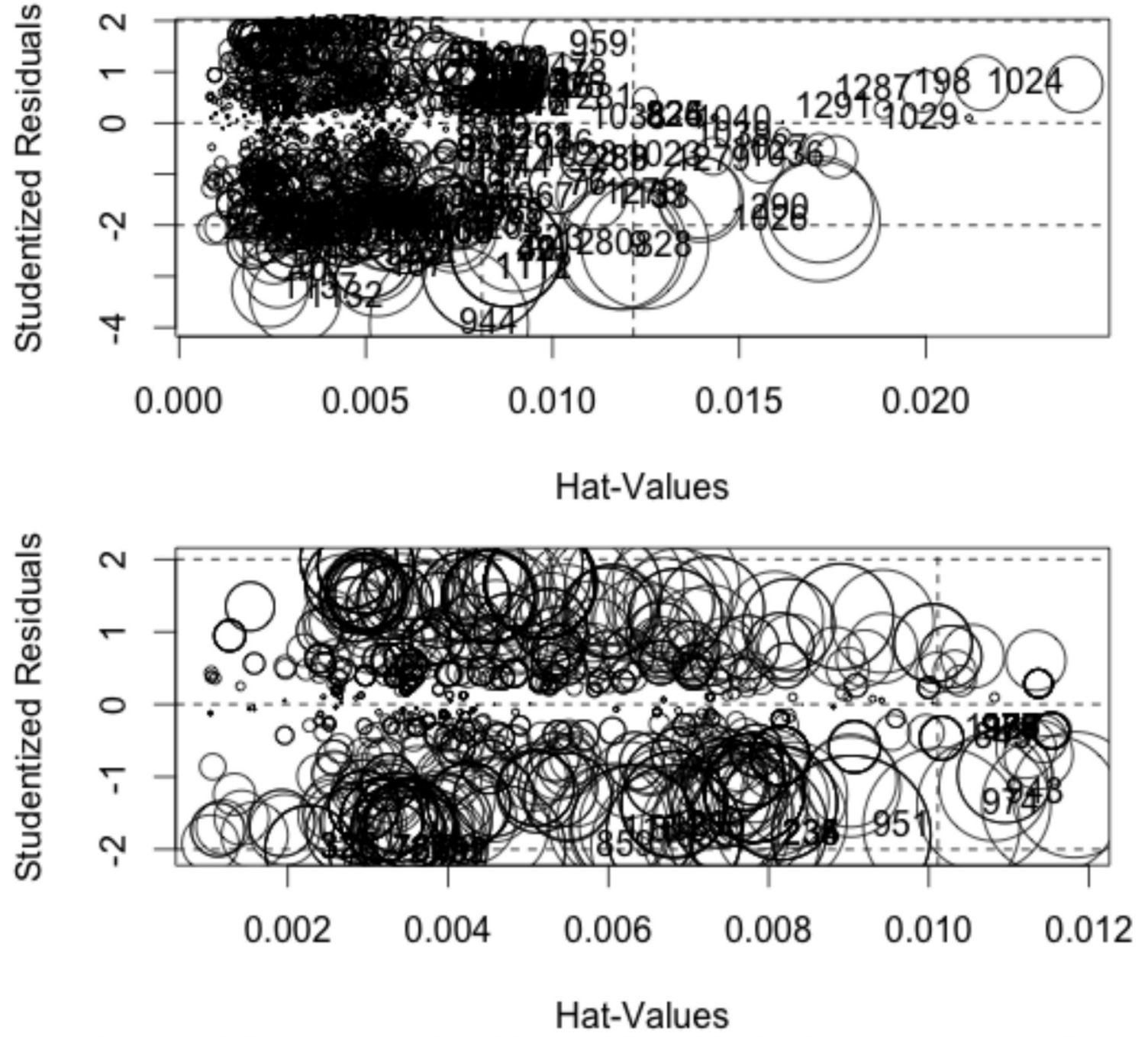

Figure 16: Influence plots of model 27 and $27^{*}$ (with 100 observations removed)

Table 30: Regression results of Model 27* (with unusual observations removed)

\begin{tabular}{lll}
\hline Variable & $\begin{array}{l}\text { Model 27 } \\
\text { (6 Months; } \mathbf{n = 1 2 2 8 )}\end{array}$ & $\begin{array}{l}\text { Model 27 } \\
\text { (6 Months; } \mathbf{n = 9 8 4 )}\end{array}$ \\
\hline Intercept & $2.1850 * * *$ & $2.84100^{* * *}$ \\
log10(Contributor.density) & -0.10918 & $-0.11365^{*}$ \\
$\log 10$ (Commit.density) & $-0.26345^{* * *}$ & $-0.29687^{* * *}$ \\
Comment.ratio & 0.05533 & $-0.43002^{*}$ \\
$\log 10($ Code.churn.ratio) & $0.15199^{* * *}$ & $0.11581^{* *}$ \\
$\mathrm{R}^{2}$ & 0.04 & 0.06 \\
\hline${ }^{*} \mathrm{p}<0.05 ;{ }^{* *}<0.01 ;{ }^{* * *} \mathrm{p}<0.001$ & & \\
\hline
\end{tabular}

Copyright (C) 2017 S. M. Monzur Murshed 


\subsubsection{Effect size}

Finally, we examine the effect size and practical significance of the associations found between exposure time (the response variable) and project properties (the explanatory variables). Our approach is to vary the explanatory variables over a practical and realistic range, and report the predicted values of the response variable.

We begin with model 7, a linear model with two explanatory variables, estimated using a 6-month time-averaging window:

[Model 7] $t_{e}=1333.20-(84.73) x_{2}-(1155.62) x_{3}$

where $x_{2}$ is commit density, and $x_{3}$ is comment ratio

Table 31 reports the results of varying each explanatory variable over the interquartile range of the data set (previously reported in Table 18), holding the other explanatory variable constant at the median value, and the results of varying both explanatory variables together over the interquartile range. 
Table 31: Effect size of model 7 (linear model, 6-month time-averaging)

\begin{tabular}{|c|c|c|c|c|c|c|c|}
\hline & \multicolumn{4}{|c|}{ Explanatory variables } & \multicolumn{3}{|c|}{ Response variable } \\
\hline & \multicolumn{2}{|c|}{$\begin{array}{l}\text { Commit density } \\
\qquad\left(x_{2}\right)\end{array}$} & \multicolumn{2}{|c|}{$\begin{array}{c}\text { Comment ratio } \\
\left(x_{3}\right)\end{array}$} & \multirow{2}{*}{$\begin{array}{l}\text { Time of } \\
\text { exposure }\end{array}$} & \multirow{2}{*}{$\begin{array}{l}\text { Difference } \\
\text { between } \\
\text { largest and } \\
\text { smallest } \\
\text { Delta }\end{array}$} & \multirow{2}{*}{\begin{tabular}{l} 
Fraction \\
of \\
median \\
value \\
\multicolumn{1}{c}{$\%$}
\end{tabular}} \\
\hline & Quartile & Value & Quartile & Value & & & \\
\hline \multirow{3}{*}{$\begin{array}{l}\text { Vary commit density; } \\
\text { comment ratio held } \\
\text { constant at median }\end{array}$} & $1^{\text {st }}$ quartile & 0.686 & median & 0.243 & 994.0 & & \\
\hline & median & 1.244 & median & 0.243 & 946.7 & 159.4 & $16.8 \%$ \\
\hline & $3^{\text {rd }}$ quartile & 2.567 & median & 0.243 & 834.6 & & \\
\hline \multirow{3}{*}{$\begin{array}{l}\text { Vary comment ratio; } \\
\text { commit density held } \\
\text { constant at median }\end{array}$} & median & 1.244 & $1^{\text {st }}$ quartile & 0.160 & 1042.7 & & \\
\hline & median & 1.244 & median & 0.243 & 946.7 & 163.1 & $17.2 \%$ \\
\hline & median & 1.244 & $3^{\text {rd }}$ quartile & 0.301 & 879.7 & & \\
\hline \multirow{3}{*}{$\begin{array}{l}\text { Vary both commit } \\
\text { density and commit } \\
\text { density over } \\
\text { interquartile range }\end{array}$} & $1^{\text {st }}$ quartile & 0.686 & $1^{\text {st }}$ quartile & 0.160 & 1090.0 & & \\
\hline & median & 1.244 & median & 0.243 & 946.7 & 322.5 & $34.1 \%$ \\
\hline & $3^{\text {rd }}$ quartile & 2.567 & $3^{\text {rd }}$ quartile & 0.301 & 767.6 & & \\
\hline
\end{tabular}

Increasing commit density from the first quartile value $(0.686)$ to the third quartile value (2.567) while holding comment ratio at the median value $(0.243)$ reduces the predicted exposure time by 159 days from 994 days to 835 days - a difference of $17 \%$ of the median. Increasing comment ratio from the first quartile value $(0.160)$ to the third quartile value (0.301) while holding commit density at the median value (1.244) reduces the predicted exposure time by 163 days from 1043 days to 880 days - a difference of $17 \%$ of the median. Varying both variables together reduces the predicted exposure time by 323 days - a difference of $34 \%$ of the median value.

For comparison, Table 18 previously reported summary statistics of exposure time for the full sample: a median exposure of 558 days, a mean exposure of 893 days. Thus, using model 7 , the predicted exposure time for median values of the explanatory variables (947 days) is $70 \%$ higher than the median exposure time of the sample, and $6 \%$ 
higher than the mean - of a distribution shown to be right-skewed.

Similarly, we repeat this approach with model 23 , a logarithmic model with two explanatory variables, estimated using a 6-month time-averaging window:

[Model 23] $\log _{10}\left(t_{e}\right)=2.8156-(1.1741) x_{1}-(0.06061) x_{2}$

$$
t_{e}=10^{(2.8156-(1.1741) x 1-(0.06061) x 2)}
$$

where $x_{1}$ is contributor density and $x_{2}$ is commit density

Table 32 reports the results of varying each explanatory variable over the interquartile range of the data set (previously reported in Table 18), holding the other explanatory variable constant at the median value, and the results of varying both explanatory variables together over the interquartile range.

Table 32: Effect size of model 23 (log response, 6-month time-averaging)

\begin{tabular}{|c|c|c|c|c|c|c|c|}
\hline & \multicolumn{4}{|c|}{ Explanatory variables } & \multicolumn{3}{|c|}{ Response variable } \\
\hline & \multicolumn{2}{|c|}{$\begin{array}{l}\text { Contributor density } \\
\qquad\left(x_{1}\right)\end{array}$} & \multicolumn{2}{|c|}{$\begin{array}{l}\text { Commit density } \\
\qquad\left(x_{2}\right)\end{array}$} & \multirow{2}{*}{$\begin{array}{l}\text { Time of } \\
\text { exposure } \\
\text { Predicted }\end{array}$} & \multirow{2}{*}{$\begin{array}{l}\text { Difference } \\
\text { between } \\
\text { largest and } \\
\text { smallest } \\
\text { Delta }\end{array}$} & \multirow{2}{*}{\begin{tabular}{l} 
Fraction \\
of \\
median \\
value \\
\multicolumn{1}{c}{$\%$}
\end{tabular}} \\
\hline & Quartile & Value & Quartile & Value & & & \\
\hline \multirow{3}{*}{$\begin{array}{l}\text { Vary contributor } \\
\text { density; commit } \\
\text { density held constant } \\
\text { at median }\end{array}$} & $1^{\text {st }}$ quartile & 0.017 & median & 1.244 & 525.1 & & \\
\hline & median & 0.032 & median & 1.244 & 504.2 & 62.7 & $12.4 \%$ \\
\hline & $3^{\text {rd }}$ quartile & 0.064 & median & 1.244 & 462.4 & & \\
\hline \multirow{3}{*}{$\begin{array}{l}\text { Vary commit density; } \\
\text { contributor density } \\
\text { held constant at } \\
\text { median }\end{array}$} & median & 0.032 & $1^{\text {st }}$ quartile & 0.686 & 545.1 & & \\
\hline & median & 0.032 & median & 1.244 & 504.2 & 125.8 & $25.0 \%$ \\
\hline & median & 0.032 & $3^{\text {rd }}$ quartile & 2.567 & 419.2 & & \\
\hline \multirow{3}{*}{$\begin{array}{l}\text { Vary both contributor } \\
\text { density and commit } \\
\text { density over } \\
\text { interquartile range }\end{array}$} & $1^{\text {st }}$ quartile & 0.017 & $1^{\text {st }}$ quartile & 0.686 & 567.6 & & \\
\hline & median & 0.032 & median & 1.244 & 504.2 & 183.1 & $36.3 \%$ \\
\hline & $3^{\text {rd }}$ quartile & 0.064 & $3^{\text {rd }}$ quartile & 2.567 & 384.5 & & \\
\hline
\end{tabular}


Increasing contributor density from the first quartile value $(0.017)$ to the third quartile value (0.064) while holding commit density at the median value (1.244) reduces the predicted exposure time by 63 days from 525 days to 462 days - a difference of $12 \%$ of the median. Increasing commit density from the first quartile value $(0.686)$ to the third quartile value (2.567) while holding contributor density at the median value $(0.032)$ reduces the predicted exposure time by 126 days from 545 days to 419 days - a difference of $25 \%$ of the median. Varying both variables together reduces the predicted exposure time by 183 days - a difference of $36 \%$ of the median value.

The predicted exposure time of model 23 for median values of the explanatory variables (504 days) is 10\% lower than the median exposure time of the sample (558 days), and $44 \%$ higher than the mean (893 days).

In summary, varying the explanatory variables over a practical and realistic range is associated with reduction in predicted exposure time on the order of months $(10.8$ months for model 7, and 6.1 months for model 23) - a difference of practical significance to practitioners.

The chapter has presented of the results of the thesis. The next chapter discusses these results. 
An investigation of software vulnerabilities in open source software projects using data from publicly-available sources

Copyright (C) 2017 S. M. Monzur Murshed

\section{Discussion}

This chapter discusses the results that were reported in the previous chapter.

Section 8.1 presents the answers to the three guiding research questions. Section 8.2 reflects on the lesson learned by the author from completing this thesis. Section 8.3 discusses the contribution to research. Section 8.4 discusses the contribution to practice. Section 8.5 addresses the limitations of this research. Section 8.6 proposes opportunities for future research.

\subsection{Answers to the research questions}

The first of three guiding research questions from section 1.1 was the following: (1) How do the observable properties of an open source software project relate to the time taken to discover and fix software vulnerabilities?

First, more developer activity (in the form of commit density - the number of source code commits per month relative to the size of the software code base) is associated with shorter exposure time (between the introduction of a vulnerability and the release of a fix). This finding was robust to all modelling assumptions, statistically significant in all tests, and of a sufficiently large effect size to be significant in practice.

Second, there is some evidence - suggestive but not conclusive - that three other factors may be associated with exposure time: (1) contributor density (the number of contributors in a month relative to the size of the code base) as a measure of community participation, (2) comment ratio (the proportion of comments in the source code base) as an approach to tame complexity and ease understanding, and (3) code churn ratio (the 
proportion of source code that changes in a month) as a measure of churn. However, these findings were not robust to changes in modelling assumptions.

Third, the project properties examined here can explain a relatively small proportion of the variance in exposure time ( $\mathrm{R}^{2}$ equal to or less than $7 \%$ in all models). Further work is needed to identify and understand the main drivers of exposure time by considering other explanatory variables in addition to the four examined here (discussed in more detail in section 8.6). Although low $\mathrm{R}^{2}$ values are problematic for making precise predictions due to the high levels of unexplained "noise" in the response variables, Frost (2014) defends their value: "Even when R-squared is low, low p-values still indicate a real relationship between the significant predictors and the response variable" - a potentially insightful result for an exploratory study in a new domain.

Due to limitations in data availability, we were unable to distinguish between the time to discover a vulnerability and the time to fix a vulnerability; we could examine only the time of exposure - the combined time to discover and fix a vulnerability.

The second guiding research question was the following: (2) To what extent is the data required to answer question 1 available through publiclyavailable online sources?

Some of the data required was easily available from online databases with automated data collection procedures, some was available with difficulty through manual inspection of websites and documents, and some was not available.

Data readily harvested from online databases included the commit history of open source software projects (including monthly metrics on code, commits, and contributors), 
CVE records of vulnerabilities (including CVE IDs affecting a project, CVSS metrics, products and product version affected, and references), and additional information about CVEs (including exploits, OVAL definitions, and user-supplied information).

Data available with manual effort included project release history (needed to estimate the introduction event and the fix event using records on affected versions).

Data not readily available included the date of the vulnerability discovery, which limited the analysis performed here to only four of the twelve hypotheses developed in chapter 5 - a significant limitation further discussed in sections 8.5 and 8.6.

The third and final guiding research question was the following:

(3) How can existing publicly-available databases be improved?

Online databases, such as CVE Details and Black Duck Open Hub, were designed to deliver value to practitioners; these same systems could be readily extended to service the needs of researchers by making improvements in four areas: (1) coverage, (2) quality, (3) access, and (4) integration.

Coverage about vulnerabilities should include an accurate timeline of events in the vulnerability lifecycle (section 4.1) - about the introduction, discovery, and fix of the vulnerability. The timeline of events should be easily harvested by software scripts, and each event should be linked to supporting evidence - for example, the security bulletin associated with public disclosure. The required information may already exist in the "references" links of some CVEs, but these URLs and online documents are not easily accessed and parsed. Coverage about affected products and versions, and coverage of open source projects, should both include timelines of release history. Coverage of open 
source software projects should include security vulnerabilities in those projects.

Quality improvements are about accuracy of the data. There are known inconsistencies in CVE data (described in section 3.1) including projects and products with multiple names (confounding search results), and omissions from the list of affected versions. Correcting these errors upstream at the CVE List or the National Vulnerability Database would benefit all consumers; alternatively, these errors could be corrected by a downstream aggregator such as CVE Details. Correcting errors in old records is valuable to researchers seeking historical data sets.

Access improvements refers to the ease of data collection. CVE Details should document and expose an API. Open Hub should expand the existing Ohloh API and improve the Ohloh API documentation - for example, by providing samples of code in R. Integration improvements refers to the interoperability of online sources. Both Open Hub and CVE Details could be improved by either linking to or aggregating content from the other: for example, clicking through a CVE record to information about an open source software project, or clicking through a project record for information about CVEs. In 2016, Open Hub added a new "Project Security" dashboard alongside the code, activity, and community dashboards of each open source project (DegenPortnoy, 2016; Wilcox, 2016). Currently the security dashboard has limited functionality, including some visualizations of vulnerability counts and severity scores, and links to individual CVE records at the National Vulnerability Database. With some enhancements, including security extensions to the Ohloh API, Open Hub could become the single source for data about open source projects and vulnerabilities in those projects. 
Finally, open source projects vary widely with respect to vulnerability policy and reporting of security information on project websites. The Apache HTTP Server project, examined in section 4.2 , provides detailed security pages with vulnerability timelines that are more detailed and rigorously referenced than the corresponding CVE records. Other open source projects could provide more value to their communities by offering security pages comparable to those of the Apache HTTP Server project.

\subsection{Lessons learned from writing this thesis}

This thesis is the culmination of my graduate studies in Technology Innovation Management (TIM). From the beginning of my studies in the TIM program, I have focused on open source software and software vulnerabilities. All of my course assignments have focused on one or both of these two domains. Though my background is in mechanical engineering, not information technology, this consistency has helped me to identify a clear and relevant research question and to develop a systematic research method to answer the question. From my initial research question about understanding the relationships between project attributes, community attributes, and software vulnerabilities in open source software projects, I realized that I also had much to say about the limitations of today's online databases, and that I could now offer some of my own recommendations to help make this better.

The early days had a lot of reading. I investigated definitions of ideas like "software vulnerability", and learned how researchers define their ideas in a precise and formal way. I learned about standards, like the Common Weakness Enumeration, to help different researchers measure things in a consistent way. I learned about prominent data 
breaches and other security failures, and also about their real-life consequences. And I learned about the opinions and viewpoints of open source software developers, and realized how it might be possible to test some of their ideas.

To develop my hypotheses, I needed to keep learning. I learned about how open source software development really works, today (Fogel, 2016) and in the recent past (Fogel, 2006) - through community and meritocracy, voluntary participation and contribution, and component reuse and integration. I learned what the research on open source software had to say about architectures, complexity, community-building, release management, and participation by companies. I learned about open source metrics, about websites that collect and report metrics, and about how open source projects can differ. And I needed to learn more about cybersecurity - about code complexity, about the lifecycle of a software vulnerability, and about vendor responsibility.

To get the data to test my hypotheses, I needed to learn about techniques for automated data collection (Munzert et al. 2015), application programming interfaces (APIs), markup languages including XML and HTML, developing software scripts in R and in Python, and using an integrated development environment (RStudio; https://www.rstudio.com/). Applying what I learned, I figured out how to get data about software vulnerabilities from CVE Details, how to get data about open source projects from Black Duck Open Hub, and about how to merge these sets together into an R data frame. I had previously used R software and regression analysis in my graduate courses, but to really test my hypotheses, I needed to master applied regression and to learn new things by reading books, like Fox (2016) and Fox \& Weisberg (2011), about R packages, 
scripting, residuals, regression diagnostics, transformations, and effect size.

\subsection{Contribution to research}

This thesis makes at least three contributions to scholarly research. A first contribution is the results of the statistical hypothesis tests, which strongly support the hypothesis that higher commit density - a proxy for developer activity (Shin et al. 2011) and thus more opportunities for developer "eyeballs" to examine the source code (Raymond, 1999) - is associated with lower vulnerability exposure time. Ceteris paribus, open source projects with more commits per month to the source code are expected to find and fix vulnerabilities in less time. The purported benefits of higher levels of project activity are some of the oldest, most prominent, and frequently recurring assertions in the scholarly and practitioner literature on open source software, and this thesis reports empirical evidence supporting such assertions.

A second contribution is the conceptual framework for a vulnerability life cycle, which opens up a new research program about shortening the time to find and fix software vulnerabilities. This is complementary to the themes currently prominent in software vulnerability research, including the factors impacting vulnerability introduction and severity, policies for disclosing vulnerabilities, and the exploitation of vulnerabilities. Furthermore, it capitalizes on the perceived strengths of open source software asserted by practitioners (e.g., Free Software Foundation, 2014; Wheeler, 2015, section 2.4).

Likewise, examining factors at the project and community level is complementary to the lower levels of analysis, such as files and code commits, that are more typical in the vulnerability research reviewed in chapter 2 . The author is aware of no prior studies that 
use software release dates to proxy for events in the lifecycle of a vulnerability.

A third contribution is the workflow and software scripts, which can be used and extended by others to replicate these results, examine other variables in CVE Details and Open Hub, expand the analysis to new variables harvested from other publicly-available online sources, grow the sample to more projects, and test new hypotheses.

\subsection{Contribution to practice}

This thesis also makes at least two contributions to management practice. A first contribution is the evidence supporting an association between higher commit density and shorter software vulnerability exposure time. This association is of practical importance to producers of open source software making decisions about governance and community management, users and consumers of open source software deciding which projects to support, and to security practitioners needing to secure and test systems that consume open source software. It supports assertions in the practitioner literature, for example by Perloth (2014), Sullivan (2014), Wheeler \& Khakimov (2015), and Fogel (2016), and in the scholarly research literature, for example by West \& O’Mahony (2008) and Schweik (2013), that associate high project activity with positive outcomes.

A second contribution is the recommendations for improving publicly-available online sources provided in section 8.1. Online databases such Black Duck Open Hub, CVE Details, and the National Vulnerability Database already provide value to their target practitioner audiences. The recommendations provided here would increase the value of these databases to researchers, thus benefiting practitioners both directly and indirectly - directly through better data and more services that would also be available to 
practitioners, and indirectly through new knowledge and other outcomes of research.

Furthermore, we call upon all open source software projects to follow the example of the Apache HTTP server project (examined in section 4.2) by providing security reports at the project website that are informative, correct, and easily accessible, including the earliest release affected, the discovery event when the developers found out about the vulnerability, the disclosure event when the vulnerability was publicly disclosed, and the first release in which the vulnerability was fixed.

\subsection{Limitations}

Like all research, this thesis has limitations. Three of the mortl significant are described below.

First, without reliable data on vulnerability discovery events, there was no way to statistically test some of the more interesting hypotheses about the time to discover a vulnerability and the time to fix a vulnerability. The small sample results of section 7.2 demonstrated that the vulnerability discovery event can sometimes (but not consistently) be pinpointed from a manual inspection of the reference documents of a CVE record, but the human judgement required was beyond the capabilities of the automated data collection techniques from Munzert et al. (2015) employed here to construct the full sample data set, and thus outside the scope of this research.

Second, the quality of the qualitative results and hypothesis tests are limited by the quality of the publicly-available data sets, which have known problems described in chapter 3 and sections 7.2, 8.1, and 8.4. Section 8.1 and 8.4 provide the author's recommendations for improving the data sets. 
Third, statistical power is limited by sample size. With a larger data set of more open source projects and more vulnerabilities, the same techniques may detect smaller effect sizes. Furthermore, all projects examined here are in the same product category of content management systems. There may be unknown or unacknowledged systemic differences between CMS projects and other software project that limit the generalizability of these results.

\subsection{Future research}

There are many opportunities for future research that builds on the results of this thesis. Four of the most promising are described below.

First, there could be further analysis of the existing data set. Future work could explore polynomial relationships and other model respecifications, employ more sophisticated regression diagnostics, and do more to investigate unusual and influential observations. There could be more work done to identify and characterize the best transformations; for example, Fox (2016) recommends exploring logit transformations (i.e., the natural logarithm of "odds") and other transformation in the family of "folded powers and roots" for proportions, which may be appropriate for the comment ratio and/or code churn ratio explanatory variables. The purpose here was exploration and testing hypotheses over a range of modelling assumptions, but future work could do more to develop better estimators, perhaps using alternatives to ordinary least squares, such as robust regression (Fox, 2016, ch. 19), or non-parametric regression (Fox, 2016, ch. 18).

Second, other researchers can reuse the data collection workflow and extend the scripts to grow the data set and build new data sets. This thesis examined only a subset 
of the metrics available from Open Hub about open source software projects and communities, focusing only on the hypotheses developed in chapter 5. Other available metrics easily harvested from Open Hub include the age of the project (computed from first commit and most recent commit), the cumulative man-months of effort, the distribution licenses used, the programing languages used, and user ratings. Likewise, this thesis examined only a subset of the vulnerability metrics available from CVE Details, focusing only on the time to find and fix vulnerabilities. Other available metrics easily harvested from CVE Details include weakness type (using either the CWE ID or the CVE Details vulnerability type tags), the number of exploits, severity (using the CVSS base score or lower-level CVSS metrics), or OVAL definitions. An exploratory study, perhaps using data science techniques from "big data" problems and statistical learning, could examine the full data set to induce new propositions for future specification and testing. Many of the assertions from the scholarly and practitioner literature reported in section 2.4 were not examined here, including architecture, use and enforcement of secure coding practices, use testing tools, and the shared values held by developers; each untested assertions is an opportunity for further study.

Third, more research is needed to extract and analyze additional data about vulnerabilities. For example, a strong proxy for the discovery event would enable researchers to distinguish between the time to discover a vulnerability and the time to fix a vulnerability - some of the more interesting hypotheses developed in chapter 5, but which were not testable here with the data available. Section 7.2 reported that some information about vulnerability discover exists deep in the references of some CVE 
records, but is not easily harvested using the techniques employed here (e.g., Munzert et al. 2015). One promising approach is a traditional content analysis study to more fully characterize which data are available from CVE records and how to extract them. A second approach, building on insights from the first approach, is automated data collection and analysis with data mining, machine learning, natural language processing, and automated approaches to model selection using statistical learning (Hastie et al. 2009; Gareth et al. 2013). We urge researchers to share data sets with others, we urge online sources to provide this information in an easy-to-consume way, and we urge standards groups to incorporate more information about introduction, discovery, and fixing of vulnerabilities into the standards for capturing and sharing security information. Fourth, more research is needed on practical knowledge for practitioners. Future research could more closely examine particular open source project field settings to get closer to the phenomena of introducing, discovering, and fixing vulnerabilities, and the actionable and prescriptive knowledge about making software more secure. 
An investigation of software vulnerabilities in open source software projects using data from publicly-available sources

Copyright (C) 2017 S. M. Monzur Murshed

\section{Conclusion}

Understanding the time taken to find and fix vulnerabilities in open source software projects is of theoretical and practical importance, but is not well understood by researchers or practitioners. This thesis examined, using data harvested from online databases, the relationship between the project and community attributes of open source software projects and the time taken to discover and fix vulnerabilities in the software. The data set comprised 1268 vulnerabilities affecting the software produced by a sample of 60 open source projects in the content management system (CMS) application area. Results support an association between higher commit density (a measure of project activity) and shorter vulnerability exposure time. This thesis reports on the extent to which the data required to study vulnerabilities in open source software projects is available through publicly-available sources, and provides a set of recommendations to improve existing databases. It particular, it highlights the problems of getting good data about vulnerability discovery - when and how the open source development community learned about a vulnerability and could begin to work on a fix. Without a reliable proxy for the discovery event, the techniques used here were unable to distinguish between the time taken to discover a vulnerability and the time taken to fix a vulnerability, and could examine only the total time of exposure between introduction and fix. The data collection workflow and scripts will enable other researchers to replicate these results, to repeat the analysis with an expanded or alternative data set, and to examine other observable properties of projects and vulnerabilities. 
An investigation of software vulnerabilities in open source software projects using data from publicly-available sources

Copyright (C) 2017 S. M. Monzur Murshed

\section{References}

Anbalagan, P. 2011. A study of software security problem disclosure, correction and patching processes. Unpublished doctoral dissertation, North Carolina State University, Raleigh, North Carolina, USA.

Arbaugh, A. A., Fithen, W. L., McHugh, J. 2000. Windows of vulnerability: A case study analysis. IEEE Computer, 33(12): 52-59.

Arjun, K. C. 2012. Key factors impacting on response time of software vendors in releasing patches for software vulnerabilities. Unpublished master dissertation, University of Southern Queensland, Queensland, Australia.

Arora, A., Telan, R. and Xu, H. 2008. Optimal policy for software vulnerability disclosure. Management Science, 54(4): 642-656.

August, T., \& Tunca, T. I. 2011. Who should be responsible for software security? A comparative analysis of liability policies in network environments. Management Science, 57(5): 934-959.

Beattie, S., Arnold, S., Cowan, C., Wagle, P., \& Wright, C. 2002. Timing the application of security patches for optimal uptime. Proceedings of The 16th USENIX Systems Administration Conference: $101-110$.

Bever, L. 2014. Major bug called 'Heartbleed' exposes Internet data. The Washington Post, April 9.

https://www.washingtonpost.com/news/morning-mix/wp/2014/04/09/major-bugcalled-heartbleed-exposes-data-across-the-internet/

Bhattacharya, P., Ulanova, L., Neamtiu, I. \& Koduru, S. C. 2013. An empirical analysis of bug reports and bug fixing in open source Android apps. Proceedings of the 17th European Conference on Software Maintenance and Reengineering: 133143.

Black Duck Software. 2014. Ohloh API documentation. https://github.com/blackducksoftware/ohloh_api (accessed September 30 2016),

Bosu, A., Carver, J. C., Hafiz, M., Hilley, P. \& Janni, D. 2014a. Identifying the characteristics of vulnerable code changes: an empirical study. Proceedings of the 22nd ACM SIGSOFT International Symposium on Foundations of Software Engineering (FSE 2014): 257-268.

http://www.amiangshu.com/papers/Bosu-FSE-2014.pdf 
Bosu, A., Carver, J. C., Hafiz, M., Hilley, P., \& Janni, D. 2014b. When Are OSS Developers More Likely to Introduce Vulnerable Code Change? A Case Study. In Open Source Software: Mobile Open Source Technologies. Springer Berlin Heidelberg: 234-236.

Cavusoglu, H., Cavusoglu, H., \& Raghunathan. 2007. Efficiency of vulnerability disclosure mechanisms to disseminate vulnerability knowledge. IEEE

Transactions on Software Engineering, 33(3): 171-185.

CBC News. 2014. Heartbleed security bug: Canadian tax services back online. April 13. http://www.cbc.ca/news/business/heartbleed-security-bug-canadian-tax-servicesback-online-1.2608781

CBC News. 2015. Heartbleed bug crisis hit more government computers than previously disclosed. December 9. http://www.cbc.ca/news/politics/heartbleed-bugcyberattack-canada-government-departments-1.3355993

Cheswick, W. R., \& Bellovin, S. M. 1994. Firewalls and Internet security: Repelling the wily hacker. Addison-Wesley.

Constantin, L. 2014. Think that software library is safe to use? Not so fast! IT World, December 30. http://www.itworld.com/article/2864095/think-that-software-library-is-safe-touse-not-so-fast.html

Datta, S., Sarkar, P, Das, S., Sreshtha, S., Lade, P., and Majumder, S. 2014. How many eyeballs does a bug need? An empirical validation of Linus' Law. Proceedings of the 15th International Conference on Agile Processes in Software Engineering and Extreme Programming (XP 2014), Rome, Italy, May 26-30, 2014: 242-250.

De Ru, W. G., \& Eloff, J. H. P. 1996. Risk analysis modelling with the use of fuzzy logic, Computers \& Security, 15(3): 239-48.

Degen-Portnoy, P. 2016. Open Hub in 2016. OpenHub Blog, April 15. http://blog.openhub.net/2016/04/open-hub-in-2016/

Duc, A. N., Cruzes, D. S., Ayala, C., \& Conradi, R. 2011. Impact of stakeholder type and collaboration on issue resolution time in OSS projects. Open Source Systems: Grounding Research. IFIP Advances in Information and Communication Technology, 365: 1-16.

Ethiraj, S. K., Levinthal, D. 2004. Modularity and innovation in complex systems. Management Science, 50(2): 159-173.

Feng, Q., Kazman, R., Cai, Y., Mo, R., \& Xiao, L. 2016. Towards an architecture-centric approach to security analysis. Proceedings of the 2016 Working IEEE/IFIP Conference on Software Architecture: 221-230. 
Ferguson, N., Schneier, B., \& Kohno, T. 2010. Cryptography Engineering: Design Principles and Practical Applications. Indianapolis, IN: Wiley.

Fogel, K. 2006. Producing open source software: How to run a successful free software project. Sebastopol, CA: O'Reilly.

Fogel, K. 2016. Producing open source software: How to run a successful free software project, $2^{\text {nd }}$ edition (online). http://producingoss.com/ (accessed September 30 2016).

Foreman, P. 2009. Vulnerability management. Boca Ration, FL: Auerback Publications.

Fox, J., \& Weisberg, H.S. 2011. An R companion to applied regression, Second edition. Thousand Oaks, CA: Sage Publications.

Fox, J. 2016. Applied regression analysis and generalized linear models, Third edition. Thousand Oaks, CA: Sage Publications.

Free Software Foundation statement on the GNU Bash "shellshock" vulnerability. September 25, 2014.

https://www.fsf.org/news/free-software-foundation-statement-on-the-gnu-bashshellshock-vulnerability

Frei, S. 2009. Security Econometrics The Dynamics of (In) Security. Unpublished doctoral dissertation, Eth zurich, ETH Zurich, Switzerland.

Frei, S., May, M., Fiedler, U., \& Plattner, B. 2006. Large-scale vulnerability analysis. Proceedings of the 2006 SIGCOMM workshop on Large-scale attack defense: 131-138.

Frei, S., Schatzmann, D., Plattner, B., \& Trammel, B. 2009. Modelling the security ecosystem - The dynamics of (in)security. Workshop on the Economics of Information Security (WEIS), London, UK.

Frost, J. 2012. How to interpret a regression model with low R-squared and low $p$ values. The Minitab Blog, June 12.

http://blog.minitab.com/blog/adventures-in-statistics-2/how-to-interpret-aregression-model-with-low-r-squared-and-low-p-values

Gareth, J., Witten, D., Hastie, T., \& Tibshirani, R. 2013. An introduction to statistical learning: With applications in $\boldsymbol{R}$. New York, Springer.

Graves, T. L., Karr, A. F., Marron, J. S., \& Siy, H. 2000. Predicting fault incidence using software change history. IEEE Transactions on Software Engineering, 26(7): 653-661. 
Gegick, M., Williams, L., Osborne, J., \& Vouk, M. 2008. Prioritizing software security fortification through code-level metrics. Proceedings of the Fourth ACM Workshop on Quality of Protection: 31-38.

Hastie, T., Tibshirani, R., \& Friedman, J. 2009. The elements of statistical learning, 2nd edition. New York, Springer.

Hewlett Packard. 2013. Reducing security risks from open source software: Five steps to open source peace of mind. Technical white paper, 4AA0-8061ENW, October, Rev. 1.

Ioannidis, C., Pym, D., \& Williams, J. 2012. Information security trade-offs and optimal patching policies. European Journal Of Operational Research, 216(2): 434-444.

Joh, H. 2011. Quantitative analyses of software vulnerabilities. Unpublished doctoral dissertation, Colorado State University, Fort Collins, Colorado, USA.

Jones, J. R. 2007. Estimating Software Vulnerabilities. IEEE Security \& Privacy, 28-32. http://www.cs.colostate.edu/ malaiya/635/09/JonesEstimatingVulnerabilities.pdf

Kaner, C., Falk, J., \& Nguyen, H. Q., 1999. Testing Computer Software. Wiley.

Kerner, S. M. 2015. Why all Linux (security) bugs aren't shallow: With Heartbleed and Shellshock, the open source community realized the Linus' law can be challenged. Esecurity Planet. February 20.

http://www.esecurityplanet.com/open-source-security/why-all-linux-securitybugs-arent-shallow.html

Kim, P. 2014. The hacker playbook: Practical guide to penetration testing. North Charleston, SC: Secure Planet LLC.

Krsul, I. V. 1998. Software vulnerability analysis. Unpublished doctoral dissertation, Purdue University, West Lafayette, Indiana.

Lewis, N. 1988. Using binary schemas to model risk analysis. Proceedings of the 1988 Computer Security Risk Management Model Builders Workshop: 35-48.

Longley, D., \& Shain, M. 1987. Data and computer security dictionary of standards, concepts, and teams. Stockton Press.

MacCormack, A., Verganti, R. \& Iansiti, M. 2001. Developing products on "Internet Time": The anatomy of a flexible development process. Management Science, 47: 133-150.

Marconato, G. V., Kaaniche, M. \& Nicomette, V. 2013. A vulnerability life cycle-based security modeling and evaluation approach. The Computer Journal, 56(4): $422-$ 439. 
Massacci, F., \& Nguyen, V. H. 2010. Which is the right source for vulnerability studies? An empirical analysis on Mozilla Firefox. MetriSec2010, September 15. https://www.researchgate.net/publication/44002373_Which_is_the_Right_Source _for_Vulnerability_Studies_An_Empirical_Analysis_on_Mozilla_Firefox

Mayerfeld, H. 1988. Definition and identification of assets as the basis for risk management. Proceedings of the 1988 Computer Security Risk Management Model Builders Workshop: 21-34.

McCabe, T.J. 1976. A complexity measure. IEEE Transactions on Software Engineering. 2(4): 308-320.

McGraw, G. 2006. Software security: Building security in. Upper Saddle River, NJ: Addison-Wesley.

Meneely, A., \& Williams, L. 2009. Secure open source collaboration: An empirical study of Linus' Law. Proceedings of the 16th ACM Conference on Computer and Communication Security: 453-462.

Meneely, A., \& Williams, L. 2010. Strengthening the empirical analysis of the relationship between Linus' Law and software security. Proceedings of the 2010 ACM-IEEE International Symposium on Empirical Software Engineering and Measurement (ESEM'10).

Meneely, A., Srinivasan, H., Musa, A., Rodriguez Tejeda, A., Mokary, M., \& Spates, B. 2013. When a patch goes bad: Exploring the properties of vulnerability contributing commits. Proceedings of the 23013 ACM/IEEE International Symposium on Empirical Software Engineering and Measurement: 65-74.

Meneely, A., Rodriguez Tejeda, A. C., Spates, B., Trudeau, S., Neuberger, D., Whitlock, K., Ketant, C., \& Davis, K. 2014. An empirical investigation of socio-technical code review metrics and security vulnerabilities. Proceedings of the 6th International Workshop on Social Software Engineering: 37-44.

Merkow, M. S., \& Raghavan, L. 2012. Secure and resilient software: Requirements, test cases, and testing methods. Boca Raton, FL: CRC Press.

Microsoft (no date). Definition of a security vulnerability. https://technet.microsoft.com/en-us/library/cc751383.aspx (accessed September 30 2016).

Mitra, S., \& Ransbotham, S. 2015. Information disclosure and the diffusion of information security attacks. Information Systems Research, 26(3): 565-584. 
MITRE Corporation. 2016a. Frequently asked questions (updated November 4 2016). Common vulnerabilities and exposures. https://cve.mitre.org/about/terminology.html (accessed December 1 2016).

MITRE Corporation. 2016b. Terminology (updated April 28 2016). Common vulnerabilities and exposures. https://cve.mitre.org/about/terminology.html (accessed September 30 2016).

MITRE Corporation. 2016c. About CVE (updated September 13 2016). Common vulnerabilities and exposures. https://cve.mitre.org/about/terminology.html (accessed December 1 2016).

MITRE Corporation. 2016d. About CVE (updated June 1 2016). CVE numbering authorities. https://cve.mitre.org/cve/cna.html (accessed June 12016 ).

Möller, B., Duong, T., Kotowicz, K. 2014. This POODLE bites: Exploiting the SSL 3.0 fallback, Security Advisory. https://www.openssl.org/ bodo/ssl-poodle.pdf

Mosteller, F., \& Tukey, J. W. 1977. Data analysis and regression. Reading, MA: Addison-Wesley.

Muegge, S. 2013. Platforms, communities, and business ecosystems: Lessons learned about technology entrepreneurship in an interconnected world. Technology Innovation Management Review, February: 5-15.

Muegge, S. M. 2011. Institutions of participation: A nested case study of company participation in the Eclipse Foundation, community, and business ecosystem. Doctoral dissertation, Carleton University, Ottawa, CA.

Muegge, S., \& Weiss, M. 2010. Open source software projects as opportunities for student learning and value creation. Proceedings of the International Conference on Education and New Learning Technologies (EDULEARN10), Barcelona, Spain, July 5-7.

Munzert, S., Rubba, C, MeiBner, P., \& Nyhuis, D. 2015. Automated data collection with R: A practical guide to web scraping and text mining. Wiley.

Nagappan, N., \& Ball, T. 2005. Use of Relative Code Churn Measures to Predict System Defect Density. Proceeding of the 27th International Conference on Software Engineering, May: 284-292.

Neuhaus, S., Zimmermann, T., Holler, C., \& Zeller, A. 2007. Predicting Vulnerable Software Components. Proceedings of the 14th ACM Conference on Computer and Communications Security: 529-540. 
Nizovtsev, D., \& Thursby, M. 2007. To disclose or not? An analysis of software user behavior. Information Economics and Policy, 19: 43-64.

Okamura, H., Tokuzane, M., \& Dohi, T. 2009. Optimal Security Patch Release Timing Under Non-Homogeneous Vulnerability-Discovery Process. Proceedings of the 20th International Symposium on Software Reliability Engineering: 120-128.

OWASP. 2016. Category: Vulnerability (updated June 6 2016). https://www.owasp.org/index.php/Category:Vulnerability (accessed September 30 2016).

Özkan, S. 2012. CVEdetails.com. BlackHat Europe 2012, Amsterdam, The Neatherlands, March 14-16. https://media.blackhat.com/bh-eu-12/Arsenal/bh-eu-12-Ozkan-cvedetails.com.pdf

Ozment, A. 2007. Improving Vulnerability Discovery Models. QoP'07: Proceeding of the 2007 ACM workshop on Quality of protection: 6-11.

Perlroth, N. 2014a, Heartbleed highlights a contradiction in the web. The New York Times, April 18. http://www.nytimes.com/2014/04/19/technology/heartbleed-highlights-acontradiction-in-the-web.html

Perlroth, N. 2014b, Security experts expect 'Shellshock' software bug in Bash to be significant. The New York Times, September 25.

https://www.nytimes.com/2014/09/26/technology/security-experts-expectshellshock-software-bug-to-be-significant.html

Pfleeger, C. P., Pfleeger, S. L., \& Margulies. 2015. Security in Computing, Fifth edition. Prentice-Hall.

Plate, H., Ponta, S. E., \& Sabetta, A. 2015. Impact assessment for vulnerabilities in opensource software libraries, SAP Labs, France. http://arxiv.org/pdf/1504.04971v2.pdf

R Core Team. 2016. R: A language and environment for statistical computing, Version 3.3.2 (2016-10-31). Vienna, Austria: R Foundation for Statistical Computing. https://cran.r-project.org/doc/manuals/fullrefman.pdf

Ransbotham, S., Mitra, S., \& Ramsey, J. 2012. Are markets for vulnerabilities effective? MIS Quarterly, 36(1): 43-64.

Raymond, E. S. 1999. The cathedral and the bazaar: Musings on Linux and Open Source by an accidental revolutionary. Cambridge, MA: O'Reilly.

Rescorla, E. 2005. Is finding security holes a good idea? IEEE Security \& Privacy, 3(1): 14-19. 
Rahimi, S., \& Zargham, M. 2013. Vulnerability scrying method for software vulnerability discovery prediction without a vulnerability database. $\boldsymbol{I E E E}$ Transactions on Reliability, 62(2): 395-407.

Ransbotham, S. 2010. An empirical analysis of exploitation attempts based on vulnerabilities in open source software. Workshop on the economics of Information security: 1-25.

Roumani, Y. 2012. Predicting vulnerability risks using software characteristics. Unpublished doctoral dissertation, Kent State University, Kent, Ohio, United State.

Sargut, G., \& McGrath, R. G. 2011. Learning to live with complexity. Harvard Business Review. September: 69-76.

Schiffman, M. 2007. A complete Guide to the Common Vulnerability Scoring System (CVSS). http://www.first.org/cvss/cvss-guide.html (accessed September 30 2016).

Schneier, B. 2000. Full disclosure and the window of exposure. Crypto-Gram, September 15. https://www.schneier.com/crypto-gram/archives/2000/0915.html

Schneier, B. 2003. Beyond fear: Thinking sensibly about security in an uncertain world. Springer-Verlag.

Schweik, C. M. 2013. Sustainability in open source software commons: Lessons learned from an empirical study of SourceForge projects. Technology Innovation Management Review, 3(1): 13-19. http://timreview.ca/article/645

Schryen, G. 2011. Is open source security a myth? Communications of the ACM, 54(5): $130-140$.

Schryen, G., \& Rich, E. 2010. Increasing software security through open source or closed source development? Empirics suggest that we have asked the wrong question. Proceedings of the 43rd International Conference on System Sciences.

Schmidt, A. 2012. At the boundaries of peer production: The organization of Internet security production in the cases of Estonia 2007 and Conficker.

Telecommunications Policies, 36: 451-461.

Schultz, C. 2012. Information security trends and issues in the Moodle E-learning platform: An ethnographic content analysis. Journal of Information Systems Education, 23(4): 359-371.

Schultz, E.E. Jr., Brown, D.S. \& Longstaff, T.A. 1990. Responding to Computer Security Incidents. Livermore, CA: Lawrence Livermore National Laboratory. 
Shahazad, M., Shafiq, M. Z. \& Liu, A. X. 2012. A large scale exploratory analysis of software vulnerability life cycles. Proceedings of the International Conference on Software Engineering, 34: 771-781.

Shin, Y., \& Williams, L. 2008a. Is complexity really the enemy of software security? Proceedings of the Fourth ACM Workshop on Quality of Protection: 47-50.

Shin, Y., \& Williams, L. 2008b. An empirical model to predict security vulnerabilities using code complexity metrics. Proceedings of the International Symposium on Empirical Software Engineering and Measurement: 315-317.

Shin, Y., Meneely, A., \& Williams, L. 2011. Evaluating complexity, code churn, and developer activity metrics as indicators of software vulnerabilities. $\boldsymbol{I E E \boldsymbol { E }}$ Transactions on Software Engineering, 37(6): 772-787.

Shrivastava, A. K., Sharma, R., \& Kapur, P. K. 2015. Vulnerability Discovery Model for a Software System Using Stochastic Differential Equation. Proceeding of the International Conference on Futuristic trend in Computational Analysis and Knowledge Management: 199-205.

Snow, D. 1988. A general model for the risk management of ADP systems. Proceedings of the 1988 Computer Security Risk Management Model Builders Workshop: 145-162.

Stallman, R. 1996. Free software definition. In J. Gay (ed.), 2002, Free software free society: Selected essays of Richard M. Stallman. Boston, MA: Soho Books, pp. 43-45.

Sullivan, J. 2014. Free software needs your vote. Free Software Foundation Bulletin, 25(November): 1-3.

Symantec Corporation. 2016. 2016 Internet Security Threat Report, Volume 21 (April). https://www.symantec.com/security-center/threat-report

Vache, G. 2009. Environment Characterization and System Modeling Approach for the Quantitative Evaluation of Security. Proceedings of the International Conference on Computer Safety, Reliability and Security, Hamburg, Germany, Springer, Lecture Notes in Computer Science 5775, pp. 89-102.

Vache, G. 2009. Vulnerability Analysis for a Quantitative Security Evaluation. Proceeding of the International Symposium on Empirical Software Engineering and Management, 15-16 October 2009, Lake Buena Vista, Florida, pp. 526-534. IEEE Computer Society.

Vasek, M., Wadleigh, J., \& Moore, T. 2016. Hacking is not random: A case-control study of webserver-compromise risk. IEEE Transactions on Dependable and Secure Computing, 13(2): 206-219. 
Verizon. 2016. Verizon 2016 Data Breach Investigations Report. http://verizonenterprise.com/verizon-insights-lab/dbir/2016/

Walden, J., Doyle, M., Welch, G.A., \& Whelan, M. 2009. Security of open source web applications. Proceedings of the International Workshop on Security Measurements and Metrics: 545 - 553.

Walden, J., Doyle, M., Lenhof, R., Murray, J., \& Plunkett, A. 2010. Impact of plugins on the security of web applications. MetriSec2010, September 15, Bolzano-Bozen, Italy.

Wang, J. A., Guo, M., Wang, H., Xia, M., \& Zhou, L. 2009. Environmental metrics for software security based on a vulnerability ontology. Proceedings of the Third IEEE International Conference on Secure Software Integration and Reliability Improvement: $159-168$.

West, J. \& O'Mahony, S. 2008. The role of participation architecture in growing sponsored open source communities. Industry and Innovation, 15(2): 145-168.

Weiss, M., \& Bailetti, T. 2015. Value of open source projects: a case for open source cybersecurity, ICE/IEEE International Technology Management Conference. http://www.sce.carleton.ca/faculty/weiss/papers/2015/weiss-ice-2015.pdf

Wilcox, L. 2016. Project security. OpenHub Blog, October 4. http://blog.openhub.net/2016/10/project-security/

Williams, J., \& Dabirsiaghi, A. 2012. The unfortunate reality of insecure libraries. Aspect Security. http://www.aspectsecurity.com/research-presentations/the-unfortunate-reality-ofinsecure-libraries

Wheeler, D. A., \& Khakimov, S. 2015. Open Source Software Projects Needing Security Investments. Institute For Defense Analyses, IDA Document D-5459, v.1.0 (June 19). https://www.coreinfrastructure.org/sites/cii/files/pages/files/pub_ida_lf_cii_07091 5.pdf

Zetter, K. 2014. There is a new vulnerability named POODLE, and it's not cute. Wired, October 14. https://www.wired.com/2014/10/poodle-explained/ 


\subsection{Websites}

Apache HTTP Server Project. https://httpd.apache.org/

An open source software project within the Apache Software Foundation (ASF).

Black Duck Open Hub. https:/www.openhub.net/

A database of open source software projects, maintained by Black Duck Software.

Black Duck Software. https://www.blackducksoftware.com/

Maintains Black Duck Open Hub.

Center for Internet Security. https://www.cisecurity.org/

Maintains the OVAL standard.

CVE Details. https://www.cvedetails.com/

A database of software vulnerabilities that aggregates data from other sources.

CVE List. https://cve.mitre.org/cve/cve.html

The master copy of CVE records, maintained by MITRE Corporation.

Common Platform Enumeration (CPE). https://nvd.nist.gov/cpe.cfm

The CPE standard and official dictionary is maintained within the NIST National

Vulnerability Database.

Common Vulnerabilities and Exposures (CVE). https://cve.mitre.org/

The CVE standard, maintained by MITRE Corporation.

Common Vulnerability Scoring System (CVSS). https://www.first.org/cvss

The CVSS standard for severity scoring, maintained by the Forum of Incident

Response and Security Teams (FIRST).

Common Weakness Enumeration (CWE). https://cwe.mitre.org/

The CWE standard for classifying vulnerabilities, maintained by MITRE

Corporation.

Forum of Incident Response and Security Teams (FIRST). https://www.first.org/

Maintains the CVSS standard.

Free Software Definition (FSD). https://www.gnu.org/philosophy/free-sw.html

Maintained by the Free Software Foundation (FSF).

Free Software Foundation (FSF). https://fsf.org/

Maintains the Free Software Definition and certifies license compliance.

Industry Consortium for Advancement of Security on the Internet (ICASI).

http://www.icasi.org/

Maintains the CVRF standard for vulnerability reports. 
IT Security Database. http://www.itsecdb.com/

A database of OVAL definitions for software vulnerabilities.

Metasploit. https://www.metasploit.com/

An open source software project for penetration testing, including automated exploits of known software vulnerabilities, maintained by Rapid7.

MITRE Corporation. https://www.mitre.org/

Maintains the CVE List, and the CVE, CWE, and OVAL standards.

National Institute of Standards and Technology (NIST). https://www.nist.gov/

Maintains the National Vulnerability Database.

National Vulnerability Database (NVD). https://nvd.nist.gov/

The U.S. government repository of standards based vulnerability management data (including CVE records and the CPE standard and dictionary), maintained by the U.S. National Institute of Standards and Technology (NIST).

The Exploit Database.

https://www.offensive-security.com/community-projects/the-exploit-database/

A database of Metasploit exploit modules, maintained by Offensive Security.

Open Hub. See Black Duck Open Hub.

Open Source Definition (OSD). https://opensource.org/osd

Maintained by the Open Source Initiative (OSI).

Open Source Initiative (OSI). https://opensource.org/

Maintains the Open Source Definition (OSD) and certifies license compliance.

Open Vulnerability and Assessment Language (OVAL). https:/oval.mitre.org/ Maintained by MITRE Corporation.

R Project for Statistical Computing. https://www.r-project.org/

Software used in this research for data collection scripts and statistical analysis.

Rapid7. https://www.rapid7.com/

Maintains the Vulnerability \& Exploit Database.

RStudio. https://www.rstudio.com/

An integrated development environment (IDE) for the R programming language.

SourceForge. https://sourceforge.net/

A popular forge providing hosting services for open source software projects.

Vulnerability \& Exploit Database. https://www.rapid7.com/db/

A database of vulnerability exploits, maintained by Rapid7. 
An investigation of software vulnerabilities in open source software projects using data from publicly-available sources

Copyright (C) 2017 S. M. Monzur Murshed

\section{Appendix A: Data collection workflow}

This appendix explains the data collection workflow and scripts.

The style conventions are those recommended by Fox \& Weisberg (2011, pp. xxixxii), which are similar to the style conventions used elsewhere in technical writing about R, Python, and other software documents (e.g., Munzert, 2015).

- Menus, menu items, and the names of windows are set in an italic seriffont.

- The names of R packages and Python modules are in boldface.

- Input and output are printed in monospaced (typewriter) fonts, as they appear on the computer screen, with output edited for brevity and clarity. Input is distinguished from output by the presence of prompts:

$\circ \mathrm{R}$ input begins with $\mathrm{a}>$ prompt. The $>$ prompt at the beginning of the input and the + prompt which begins continuation lines are proved by $\mathrm{R}$, not typed the user.

- Python input begins with $a>>>$ prompt. The $>>>$ prompt at the beginning of the input and the ... prompt which begins continuation lines are provided by Python, not typed by the user.

Figure 17 illustrates the eight steps of the data collection workflow. 


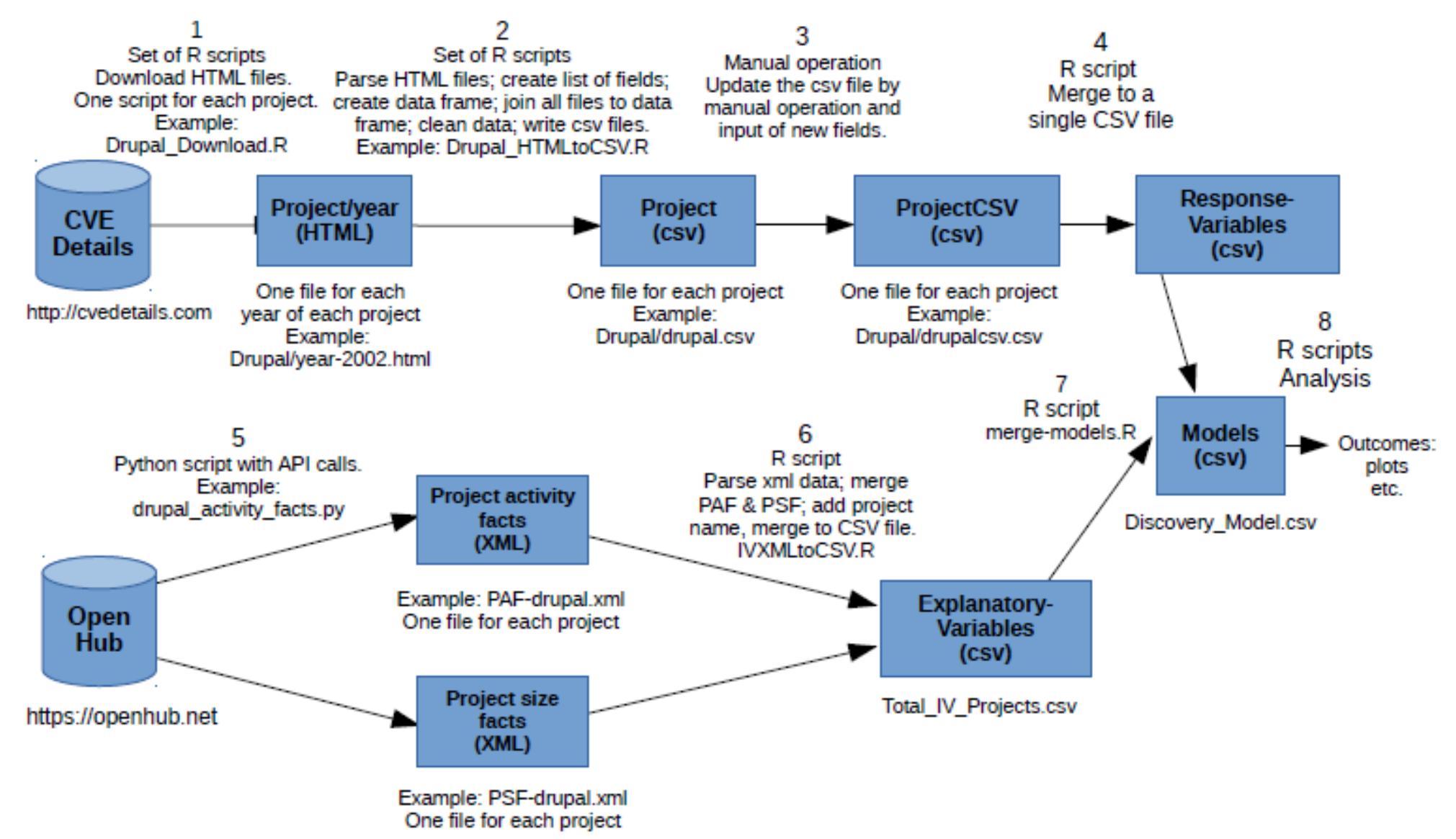

Figure 17: Data collection workflow 


\subsection{Step 0: Set-up}

- Download and install R: https://www.r-project.org/

- Install the required $\mathrm{R}$ packages by typing the following commands in the Rgui window:

> install.packages ("XML")

$>$ install.packages ("car")

$>$ install.packages ("zoo")

$>$ install.packages ("plyr")

$>$ install.packages ("stringr")

- Download and install Python: https://www.python.org/downloads/ (This theses used Python 3.5.1 for Mac OS X).

\subsection{Step 1: Get project data from CVE Details}

This step downloads HTML files from the CVE Details website and saves them to a local directory.

- Type the following command into the Rgui window:

$>$ source("RunAllProjects_Download.R")

\subsection{Step 2: Parse HTLM files}

This step parses the HTML files from the previous step and writes a comma separated value (CSV) file.

- Type the following command into the Rgui window:

> source("RunAllProjects_HTMLtoCSV.R")

\subsection{Step 3: Manual operation}

This manual step adds dates for the introduction event and the fix event.

- Open the file from step 2 using LibreOffice. (Do not use Microsoft Excel. Excel will reformat dates in ways that cause problems later when using R)

- In a web browser, navigate to the CVE Details website: www.cvedetails.com

- Go to the product search option and type a project name (e.g., "Drupal") and 
search on it

- A new window will open displaying a list of products matching the query

- Click the link of the selected project and click the link of vulnerabilities from its vulnerability statistics

- Click the link of CVE ID as listed in the project vulnerability pages

- Select the version number of the first affected version of the CVE ID from "products affected by the CVE" and copy it (CTRL-C)

- Paste (CTRL-V) the version number of the first affected version in the field of "Fst_Intro_Ver" in the same row of a CVE ID

- Find a release date for this version from various sources, such as the project website or blog, github, or Open Hub. Copy and paste or manually type the release date into the field "Introduction_Date" in the same row of the CVE ID

- Identify the version number of the first fixed version from various sources, such as the project website, or by examining the references of the CVE ID. This information is normally in the public announcement or security bulletin. Copy and paste or type the version number in the field of "1st_Fix_Ver" in the same row of the CVE ID

- Find a release date for the first fixed version from various sources, such as the project website or blog, github or Open Hub. Copy and paste or manually type the release date into the field "Fix_Date" in the same row of a the CVE ID

- Save the file as "projectnamecsv.csv" (e.g., drupalcsv.csv) in the "60CVEDetailsProject20161016" directory.

\subsection{Step 4: Join project files}

This step joins the project files from the previous step into one dataframe and outputs a CSV file "Total_DV_Projects20161024.csv" in the directory "DV".

- Type the following command into the Rgui window:

> source ("AllProjectsCVE.R")

\subsection{Step 5: Get project data from Open Hub}

This step executes a set of Python scripts to download project XML files from Open Hub 
to subdirectories within the directory python. For example, the drupal_activity_facts.py script will download the Drupal ActivityFacts XML file to the directory python/Activity_Facts. Similarly, the drupal_size_facts.py script will download the Drupal SizeFacts XML file to the directory python/Size_Facts.

- Register at www.openhub.net for an Ohloh API key. The Ohloh API documentation is posted to GitHub.

- Run each script by passing the API key and an email address as parameters. On Mac terminal, ensuring that you are in the python directory or python subdirectory and type the following:

\$/Activity_Facts/drupal_activity_facts.py Your_API_Key_Here Your_email_Āddress_Here

\subsection{Step 6: Parse and join XML files}

This step joins the Open Hub ActivityFacts and SizeFacts files for each project into one project dataframe, joins all the project data frames into one dataframe, and writes a CSV file "Total_IV_Projects.csv" in the directory IV.

- Type the following command into the Rgui window:

> source ("IV/IVXMLtoCSV.R")

\subsection{Step 7: Merge to one data frame}

This step merges the CVE Details files from step 4 with the Open Hub files from step 6 into one data frame and writes a file called "Model20161019.csv" ready for analysis.

- Type the following command into the Rgui window:

$>$ source ("Model20161019.R) 


\section{Appendix B: Data collection scripts}

This appendix provides the source code for some scripts that were previously documented in Appendix A.

The scripts called in Appendix A for step 1 (RunAllProjects_Download.R), step 2 (RunAllProjects_HTMLtoCSV.R), and step 4 (AllProjectsCVE.R) each call a set of lower-level scripts, one script for each project in the project sample. The scripts provided below for step 1, step 2, and step 4 are the lower-level scripts for the Drupal project, provided as examples.

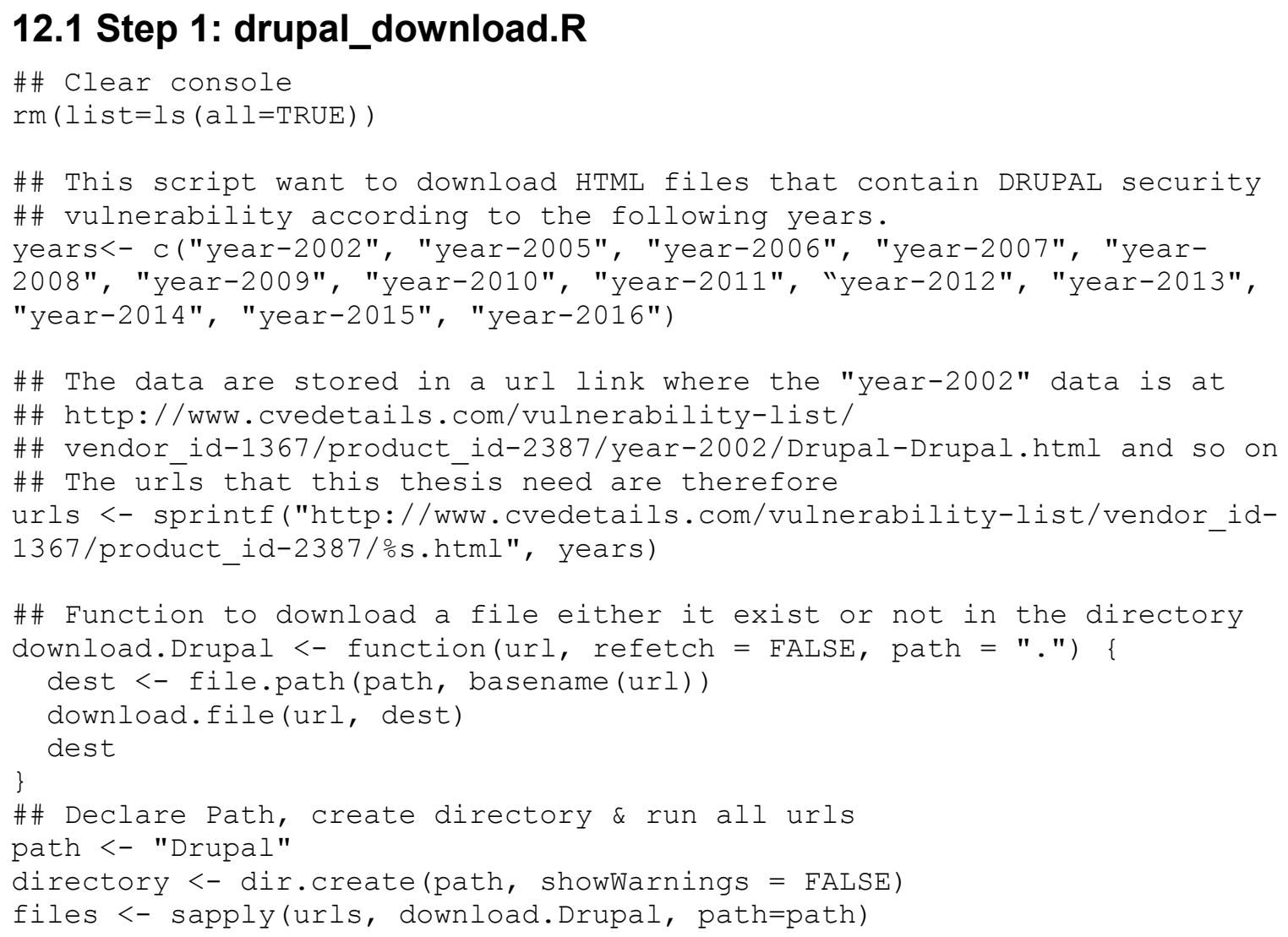




\subsection{Step 2: drupal_HTMLtoCSV.R}

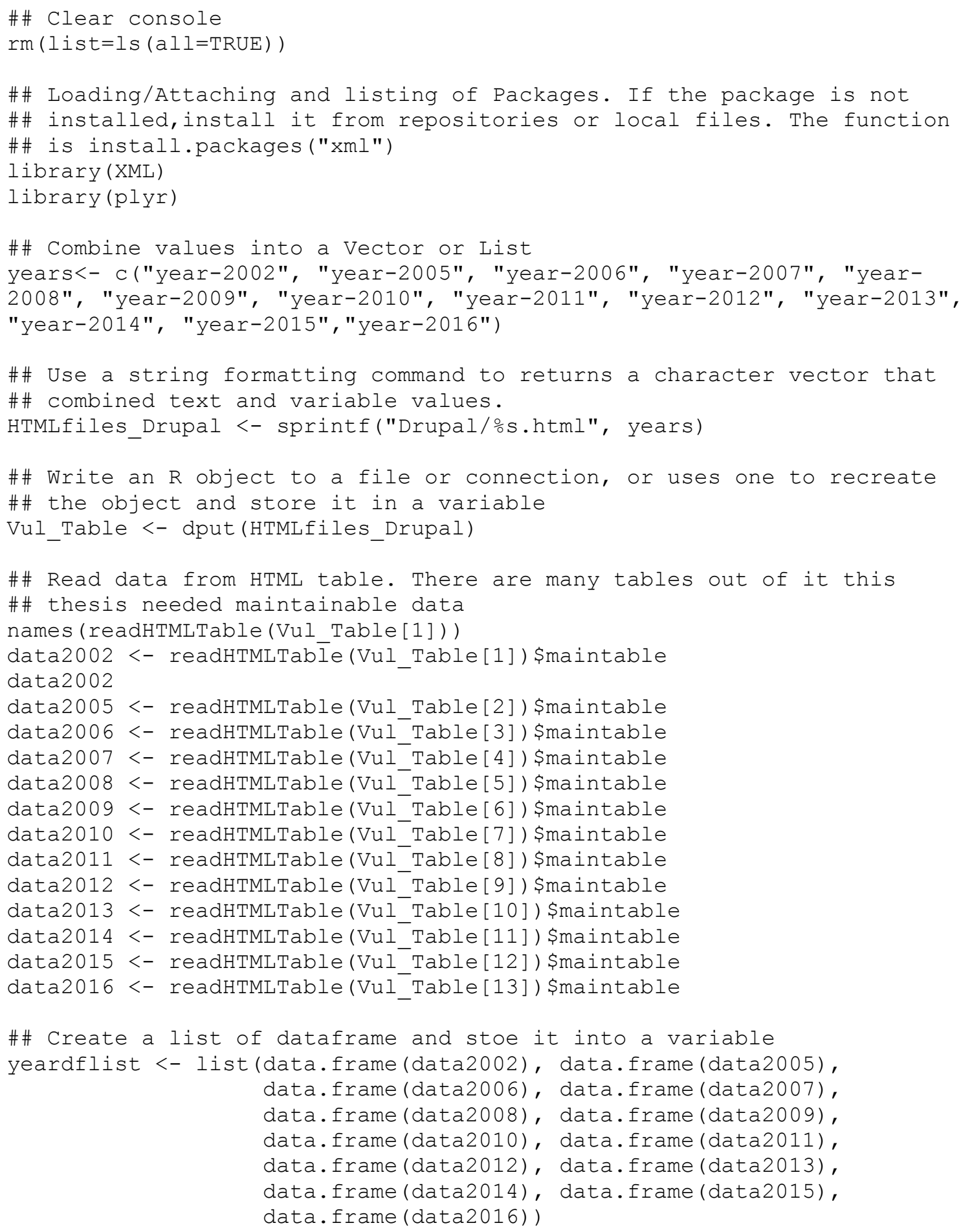

\section{Copyright (C) 2017 S. M. Monzur Murshed}




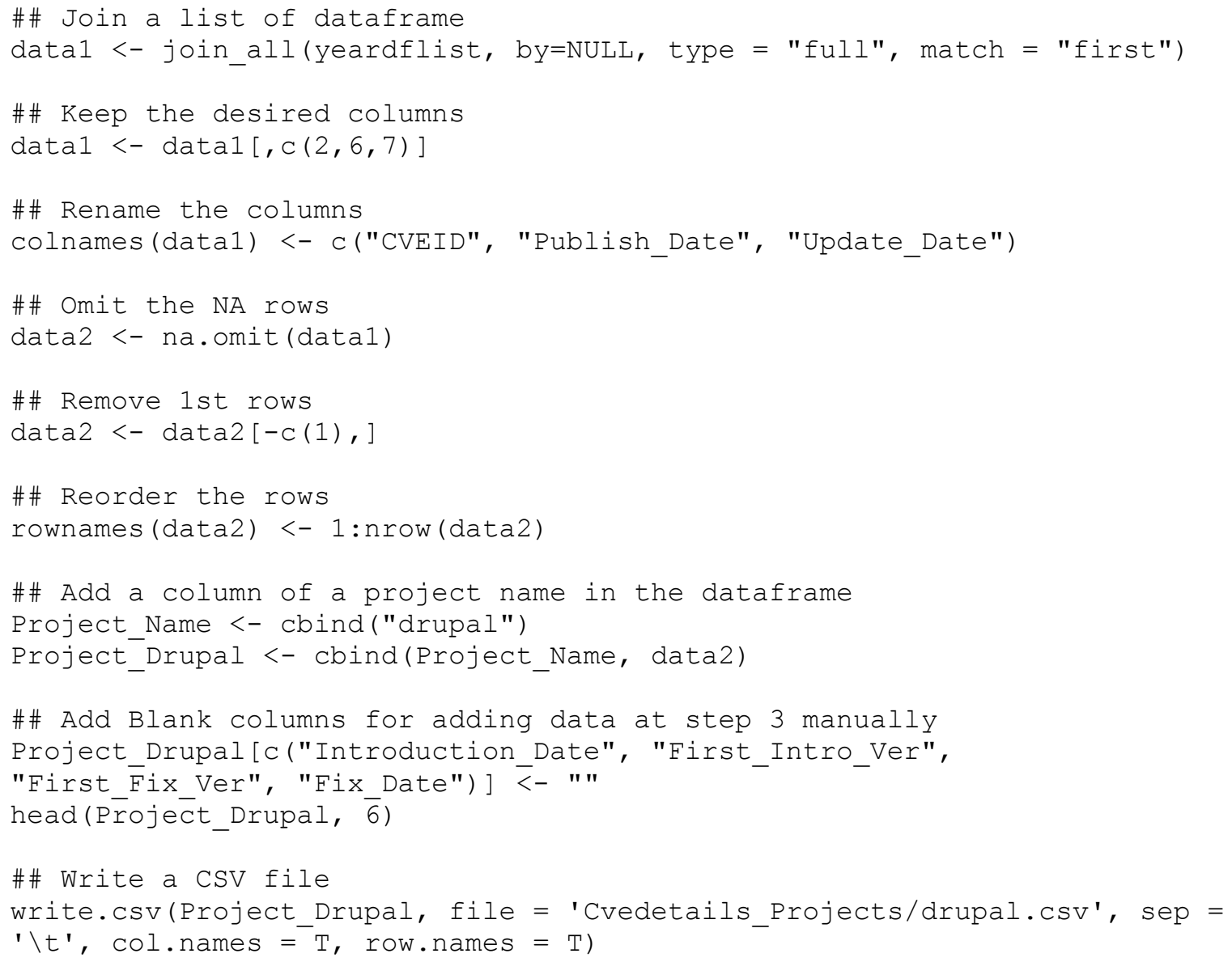

\subsection{Step 4: drupal_HTMLtoCSV.R}

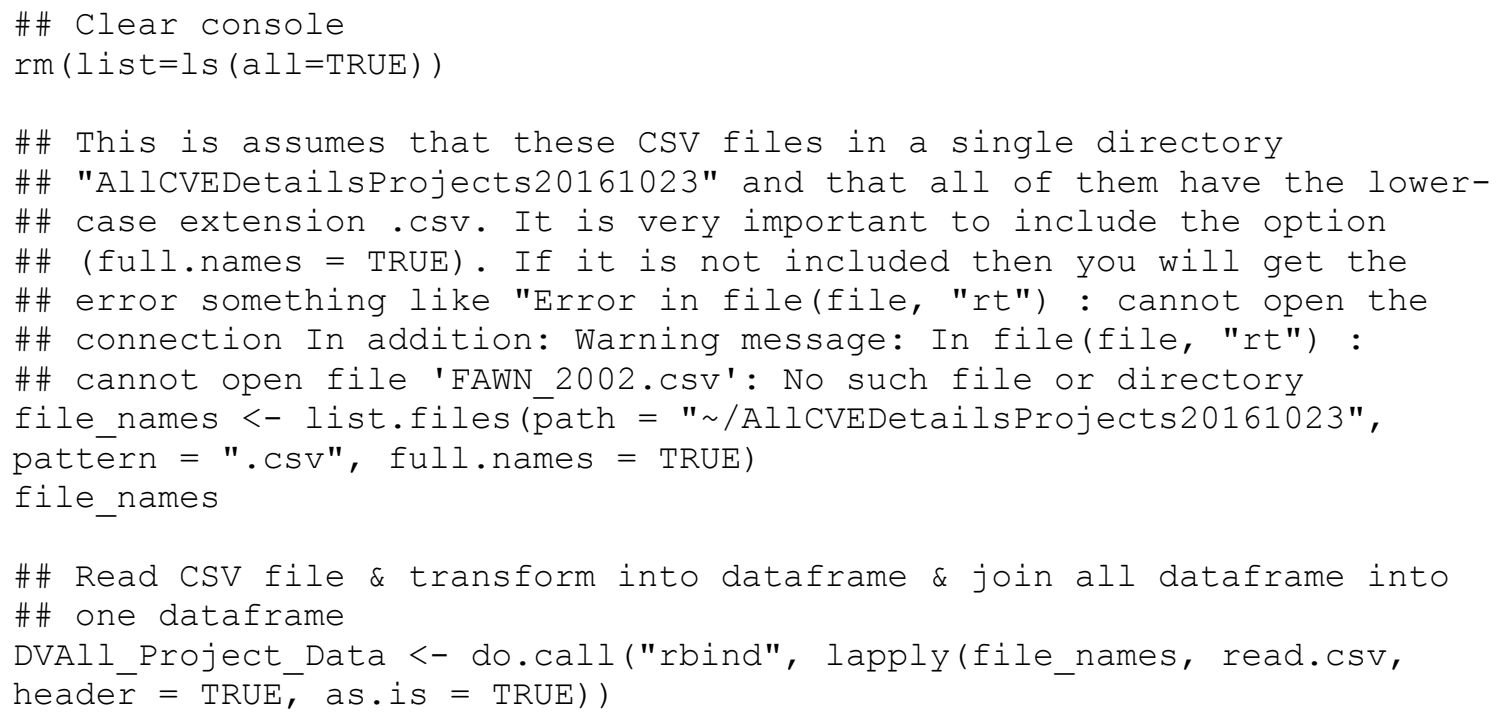




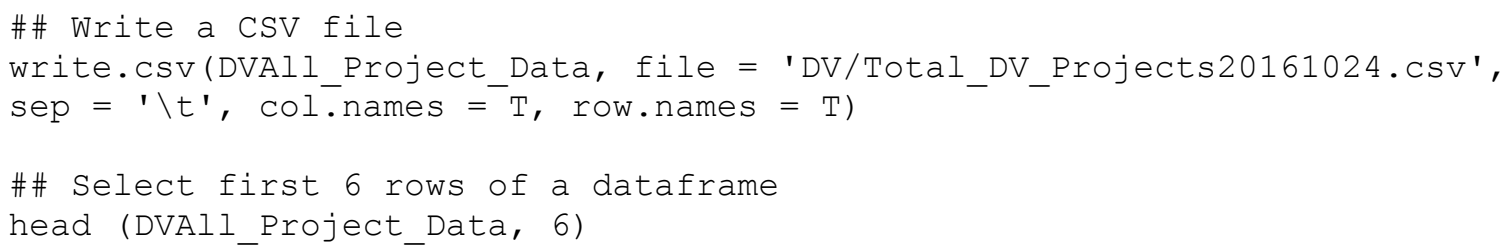

\subsection{Step 5: drupal_activity_facts.py}

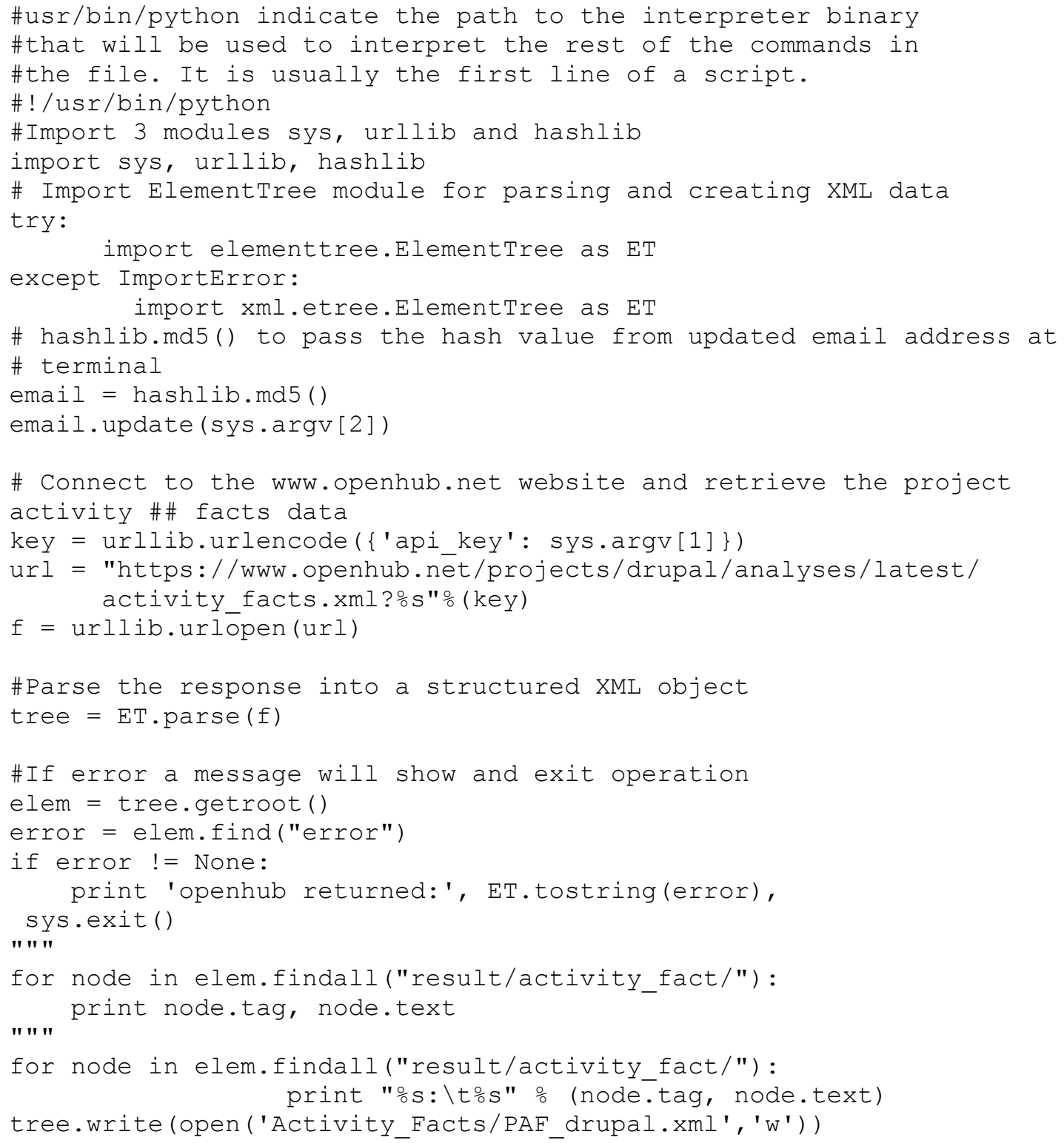

\section{Copyright (C) 2017 S. M. Monzur Murshed}

